Supporting Information

\title{
Supertwistacene: A Helical Graphene Nanoribbon
}

Shuang Ma, ${ }^{\dagger, \#}$ Jiajian Gu, ${ }^{\dagger, \#}$ Chaojun Lin, ${ }^{\dagger}$ Zhixing Luo,${ }^{\dagger}$ Yanpeng Zhu, ${ }^{\dagger}$ Jiaobing Wang ${ }^{*}, \dagger$

E-mail: wangjb5@mail.sysu.edu.cn

${ }^{\dagger}$ School of Chemistry, Sun Yat-Sen University, Guangzhou 510275, China

\#These authors contribute equally to this work.

\section{Contents}

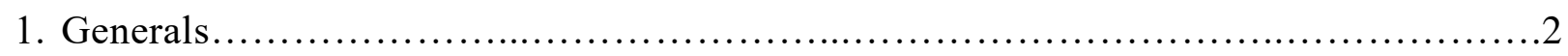

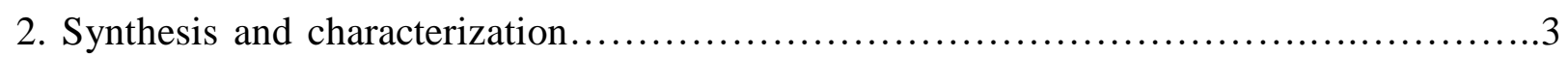

3. X-ray crystallographic studies............................................... 12

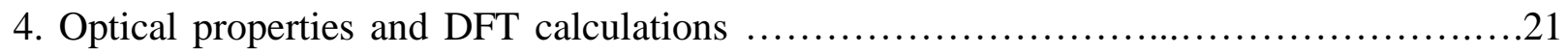

5. Electrochemical results ...................................................44

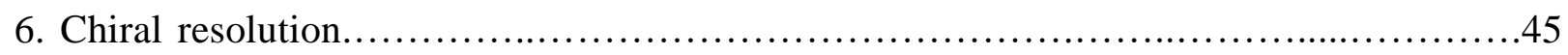

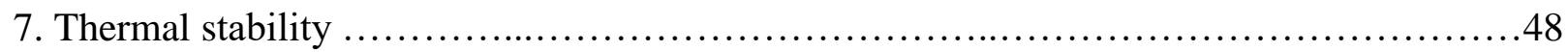

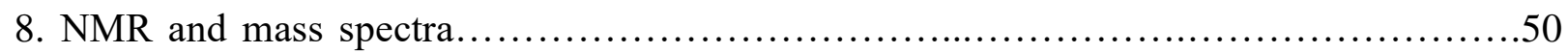

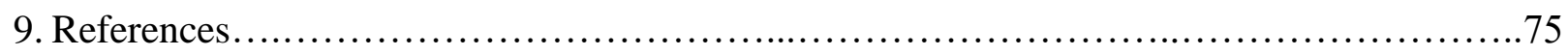




\section{Generals}

Chemicals and solvents were purchased from Energy Chemical Co., Ltd or J\&K Scientific Ltd. They are used without further purification unless otherwise noted. Dry dichloromethane (DCM) was freshly distilled over $\mathrm{P}_{2} \mathrm{O}_{5}$. Reactions were carried out with standard Schlenk technique under a $\mathrm{N}_{2}$ atmosphere. Nuclear magnetic resonance (NMR) spectra were recorded on a Bruker Avance 300 spectrometer (300 MHz). Mass spectra were measured using a Bruker ultraflex matrix assisted laser desorption-ionization time-of-flight mass spectrometer (MALDI TOF MS, supporting matrix: (E)-2-(3-(4-(t-butyl)phenyl)-2-methylallylidene)malononitrile, DCTB). Absorption, circular dichroism (CD), and emission spectra were recorded respectively on the following three instruments at $298 \mathrm{~K}$ with a $10 \mathrm{~mm}$ quartz cell: SHIMADZU UV-2600, Jasco1700, or SHIMADZU RF-5301PC. Single crystal data were collected on a XtaLAB AFC12 (RCD3) Kappa single diffractometer, an Agilent Sapphire3 Gemini Ultra single crystal diffractometer, or the Shanghai Synchrotron Radiation Facility. Chiral resolution was performed on a Shimadzu Essentia LC-16 instrument using a semi-preparative COSMOSIL cholester column $(250 \times 10 \mathrm{~mm}$ i.d $)$. Electrochemical studies were carried out with a CHI660E electrochemical workstation under a nitrogen atmosphere. 


\section{Synthesis and characterization}

\section{Synthesis of 1}

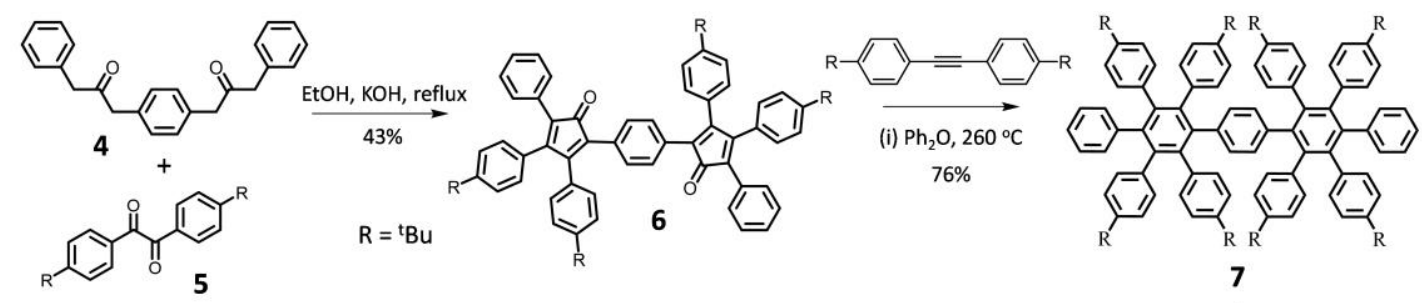

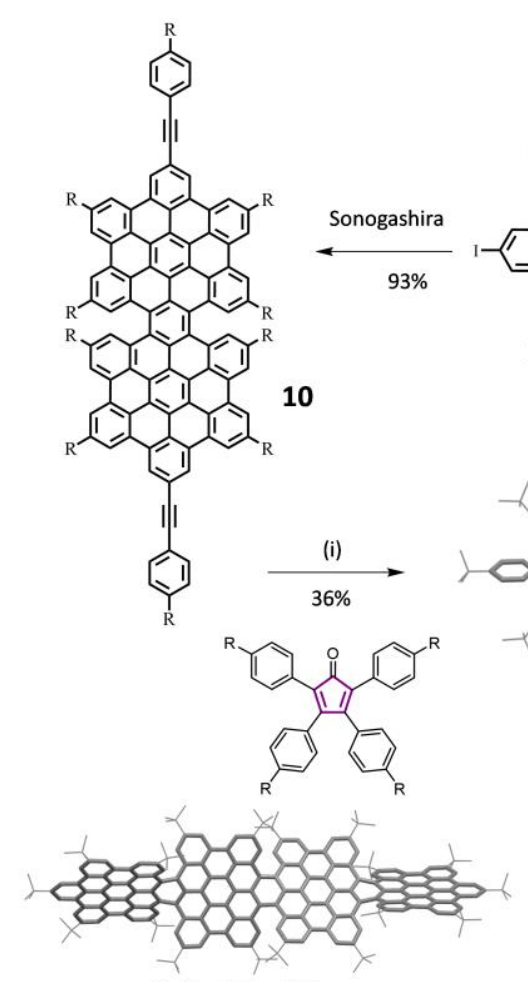

Helical-1 5\%

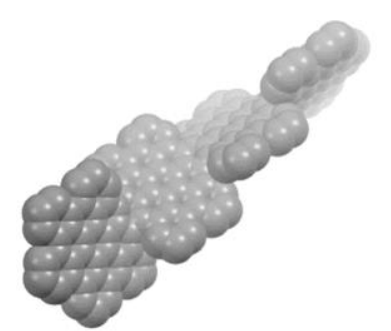

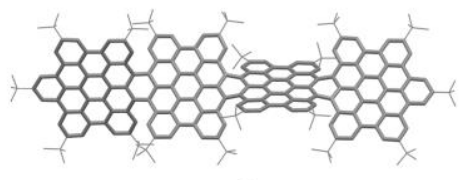

Mixed-1 $7 \%$

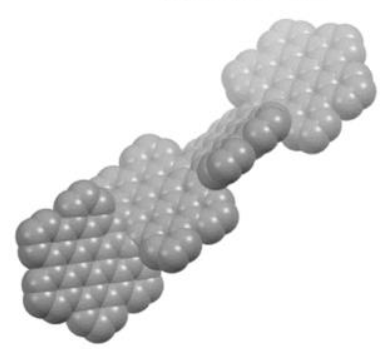

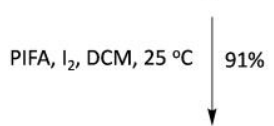
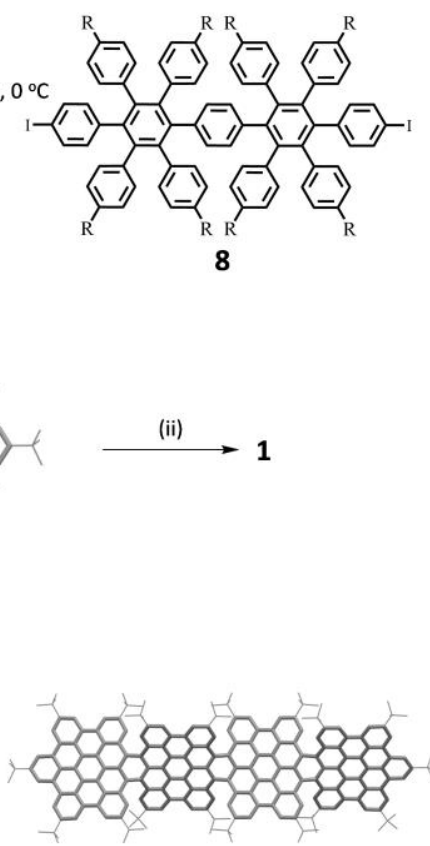

Waggling-1 $1 \%$

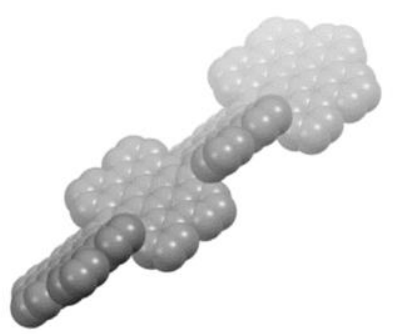

Figure S1. Synthesis of 1. Note: All three anticipated isomers of 1 were formed in the last step. Structure of Helical-1 was confirmed by single crystal X-ray diffraction. Structures of the other two isomers can be deduced from the ${ }^{1} \mathrm{H}-\mathrm{NMR}$ data (Figure S2) based on their molecular symmetry. The molecular models were optimized using molecular mechanics. $t$-Butyl groups were omitted in the space-filling presentation for clarity. 
Detailed synthesis of $\mathbf{1}$ and 6-11 was provided in the experimental section of the main text. Synthesis of $\mathbf{4}$ was shown below:

4: A mixture of 1,4-bis(bromomethyl)benzene (5.0 g, $18.9 \mathrm{mmol})$, benzyl bromide $(6.7 \mathrm{~g}, 38.8$ mmol), tetrabutylammonium bromide $(15.3 \mathrm{~g}, 47.4 \mathrm{mmol})$, and tosylmethyl isocyanide $(7.58$ $\mathrm{g}, 38.8 \mathrm{mmol})$ in DCM $(150 \mathrm{~mL})$ was stirred vigorously at RT to completely dissolve all reactants. Then aqueous $\mathrm{NaOH}(8 \mathrm{M}, 100 \mathrm{~mL})$ was added, and the reaction was stirred for $8 \mathrm{~h}$. After that, the organic phase was washed with water $(100 \mathrm{~mL} \times 3)$ and concentrated under reduced pressure. The residue was dissolved in a mixed solvent of tert-butyl methyl ether (10 $\mathrm{mL})$ and $\mathrm{DCM}(90 \mathrm{~mL})$. Then concentrated $\mathrm{HCl}(15 \mathrm{~mL}, 36 \%$, wt $/ \mathrm{wt})$ was added. The mixture was stirred for $3 \mathrm{~h}$, and quenched with saturated aqueous sodium bicarbonate $(100 \mathrm{~mL})$. The product was extracted with DCM $(100 \mathrm{~mL} \times 3)$, and the organic phase was dried over anhydrous $\mathrm{Na}_{2} \mathrm{SO}_{4}$ and concentrated under reduced pressure. The product was purified by silica gel column chromatography (DCM/MeOH, v/v, 5/1) to provide 4 as a light yellow solid (1.16 g, $18 \%$, m.p. $\left.=73-75^{\circ} \mathrm{C}\right) .{ }^{1} \mathrm{H}$ NMR $\left(300 \mathrm{MHz}, \mathrm{CDCl}_{3}\right) \delta$ 7.38-7.33 (m, 2H), 7.33-7.25 (m, 4H), 7.21-7.15 (m, 4H), 7.12 (s, 4H), 3.75 (s, 4H), 3.73 (s, 4H). $\left.{ }^{13} \mathrm{C} \mathrm{NMR} \mathrm{(75} \mathrm{MHz,} \mathrm{CDCl}_{3}\right) \delta 205.7$, 134.0, 132.9, 130.0, 129.6, 128.9, 127.2, 49.3, 48.7. MS calculated for $\mathrm{C}_{24} \mathrm{H}_{22} \mathrm{O}_{2}, 342.16$; found $365.18[\mathrm{M}+\mathrm{Na}]^{+}$.

\section{NMR and mass data of Helical-1, Mixed-1, and Waggling-1:}

Helical-1: yield 5\%, mp > $300{ }^{\circ} \mathrm{C} .{ }^{1} \mathrm{H}$ NMR $\left(300 \mathrm{MHz}, \mathrm{CDCl}_{3}\right) \delta 9.61(\mathrm{~s}, 8 \mathrm{H}), 9.52(\mathrm{~s}, 4 \mathrm{H})$, $9.46(\mathrm{~d}, 8 \mathrm{H}), 9.41(\mathrm{~s}, 4 \mathrm{H}), 9.21(\mathrm{~s}, 12 \mathrm{H}), 1.94(\mathrm{~s}, 18 \mathrm{H}), 1.93(\mathrm{~s}, 36 \mathrm{H}), 1.49(\mathrm{~s}, 36 \mathrm{H}), 1.48(\mathrm{~s}$, 36H), $1.43(\mathrm{~s}, 36 \mathrm{H}) .{ }^{13} \mathrm{C} \mathrm{NMR}\left(75 \mathrm{MHz}, \mathrm{CDCl}_{3}\right) \delta 149.5,148.5,148.4,148.4,131.3,131.2$, $131.1,131.0,130.9,130.8,130.7,130.2,129.9,127.4,127.1,126.8,126.7,126.5,126.5,124.3$, 
$124.2,121.8,121.7,121.0,120.0,119.7,119.5,119.3,119.2,119.0,36.1,36.0,35.5,35.4,32.3$, 31.9, 31.8, 31.7. MALDI-TOF MS calculated for $\mathrm{C}_{222} \mathrm{H}_{198}, 2865.55$; found, $2864.91[\mathrm{M}]^{+}$.

Mixed-1: yield 7\%, m.p. > $300{ }^{\circ} \mathrm{C} .{ }^{1} \mathrm{H}$ NMR $\left(300 \mathrm{MHz}, \mathrm{CDCl}_{3}\right) \delta 9.79(\mathrm{~s}, 2 \mathrm{H}), 9.71(\mathrm{~s}, 2 \mathrm{H})$, $9.62(\mathrm{~s}, 2 \mathrm{H}), 9.58(\mathrm{~s}, 4 \mathrm{H}), 9.54(\mathrm{~s}, 2 \mathrm{H}), 9.47(\mathrm{~s}, 8 \mathrm{H}), 9.44(\mathrm{~s}, 2 \mathrm{H}), 9.42(\mathrm{~s}, 2 \mathrm{H}), 9.27(\mathrm{~s}, 8 \mathrm{H})$, $9.24(\mathrm{~s}, 2 \mathrm{H}), 9.22(\mathrm{~s}, 2 \mathrm{H}), 1.94(\mathrm{~d}, 54 \mathrm{H}), 1.54(\mathrm{~s}, 18 \mathrm{H}), 1.50(\mathrm{~s}, 18 \mathrm{H}), 1.47(\mathrm{~s}, 36 \mathrm{H}), 1.45(\mathrm{~s}$, $36 \mathrm{H}) .{ }^{13} \mathrm{C} \mathrm{NMR}\left(75 \mathrm{MHz}, \mathrm{CDCl}_{3}\right) \delta 149.3,148.5,148.4,148.3,148.3,131.5,131.4,131.2$ 131.1, 131.0, 130.8, 130.5, 130.4, 130.4, 126.7, 126.4, 124.3, 124.2, 124.1, 121.9, 121.7, 121.6, 120.9, 119.5, 119.1, 119.0, 35.9, 35.4, 32.1, 31.8. MALDI-TOF MS calculated for $\mathrm{C}_{222} \mathrm{H}_{198}$, 2865.55; found: $2864.92[\mathrm{M}]^{+}$.

Waggling-1: yield ca. 1\%. Separation of Waggling-1 from the other side-products of the oxidation reaction was very difficult (even impracticable). Instead, pure Waggling-1 was obtained by switching the configuration of Mixed-1 with prolonged heating at high temperature $\left(260{ }^{\circ} \mathrm{C}, 40 \mathrm{~h}\right.$, see Figure S21). ${ }^{1} \mathrm{H}$ NMR $\left(300 \mathrm{MHz}, \mathrm{CDCl}_{3}\right) \delta 9.72(\mathrm{~s}, 4 \mathrm{H}), 9.68(\mathrm{~s}, 4 \mathrm{H}), 9.43$ $(\mathrm{m}, 12 \mathrm{H}), 9.30-9.22(\mathrm{~m}, 12 \mathrm{H}), 1.92(\mathrm{~s}, 54 \mathrm{H}), 1.54(\mathrm{~s}, 36 \mathrm{H}), 1.51(\mathrm{~s}, 36 \mathrm{H}), 1.45(\mathrm{~s}, 36 \mathrm{H})$. MALDI-TOF MS calculated for $\mathrm{C}_{222} \mathrm{H}_{198}, 2865.55$; found, $2864.91[\mathrm{M}]^{+}$. We do not have sufficient amount of Waggling-1 to collect its ${ }^{13} \mathrm{C}$ NMR spectrum. 

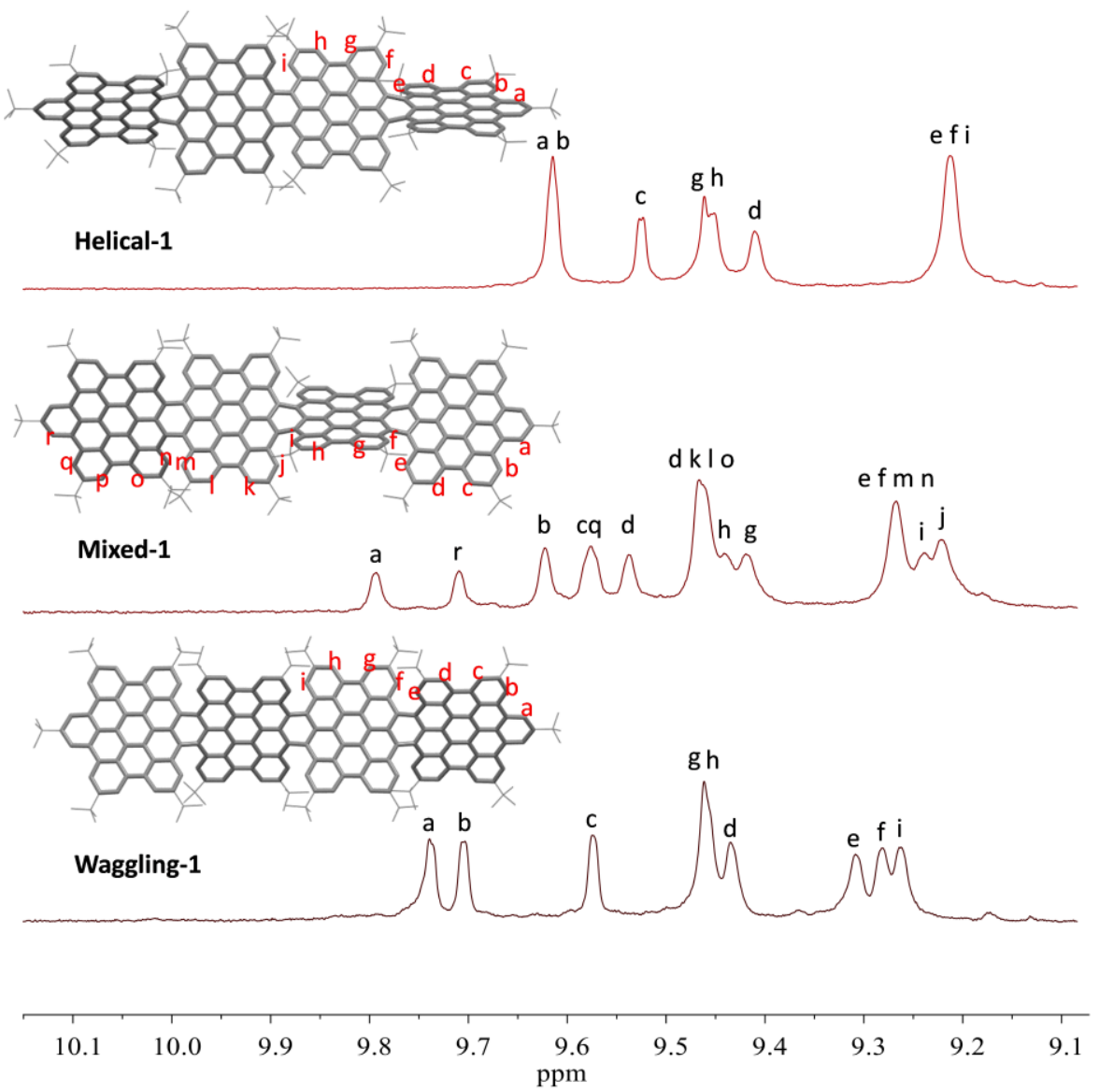

Figure S2. Partial ${ }^{1} \mathrm{H}-\mathrm{NMR}$ spectra of different isomers of $\mathbf{1}$ in $\mathrm{CDCl}_{3}$. Note: Assignment of the NMR signals for Mixed-1 and Helical-1 was based on various 2D NMR techniques. But we do not have sufficient amount of Wagging-1 to perform these measurements. Therefore, assignment of its resonance signals was based on a comparison of its ${ }^{1} \mathrm{H}-\mathrm{NMR}$ spectrum with those of Helical-1, Mixed-1, and meso-2 (Figure S4). 


\section{Synthesis of 2}

During the course of this work, 2 and its diastereoisomer (meso-2) were described by Campaña and co-workers. Our synthetic procedure was in keeping with their method (Angew. Chem., Int. Ed. 2020, 59, 7139-7145), with slight changes in yields. Therefore, detailed synthesis and characterization of $\mathbf{2}$ were not presented. We confirmed the identity of $\mathbf{2}$ with single crystal Xray diffraction (Figure S8).

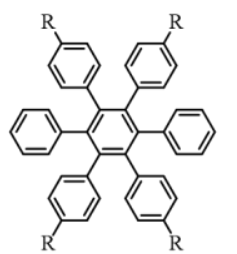

12
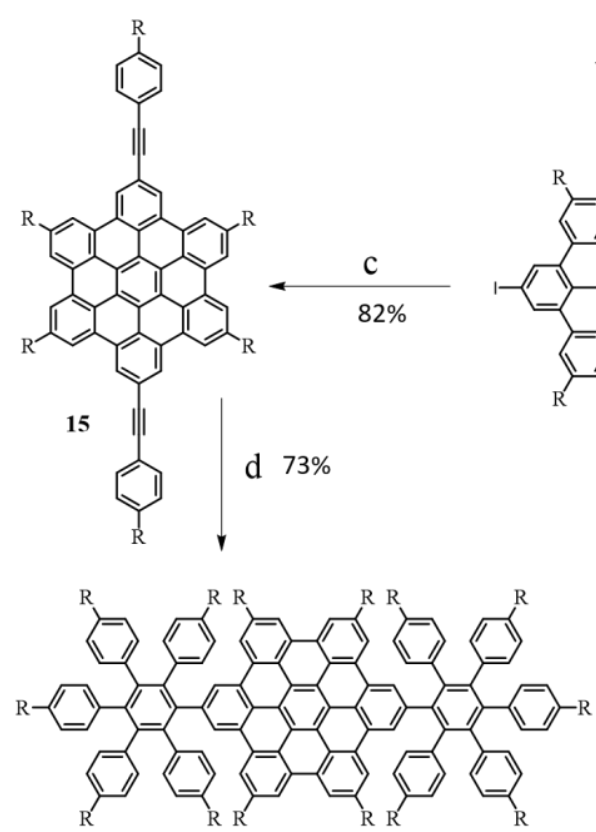

16

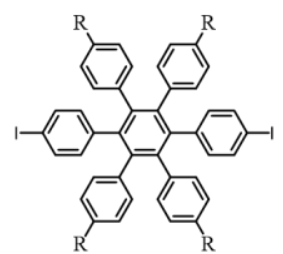

13

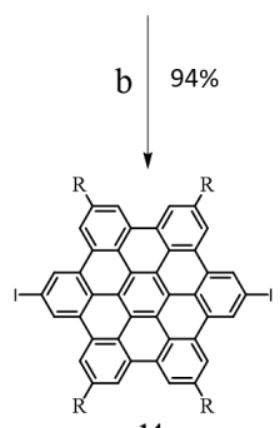

14
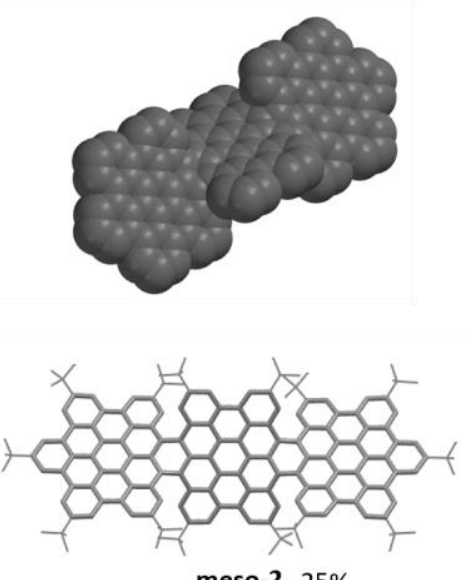

meso-2 25\%
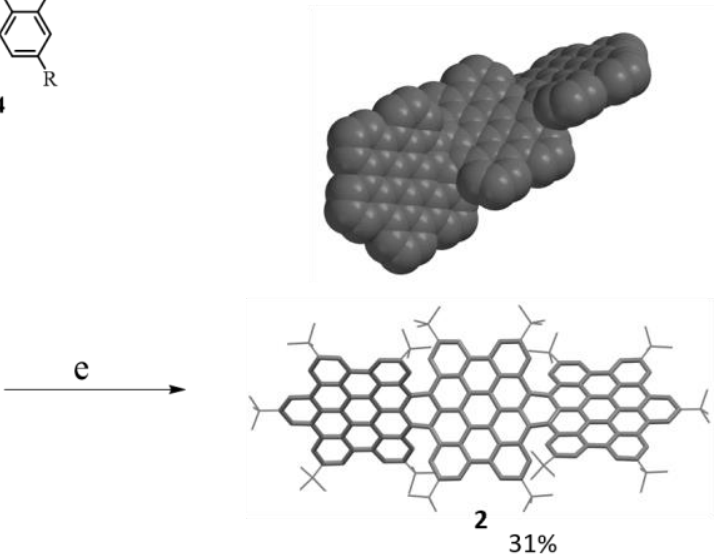

Figure S3. Reagents and conditions: a) PIFA, $\mathrm{I}_{2}$, DCM, RT, $\mathrm{N}_{2}, 4 \mathrm{~d}$; b) DDQ, TfOH, DCM, $\mathrm{N}_{2}, 0{ }^{\circ} \mathrm{C}, 3.5 \mathrm{~h}$; c) 1-(tert-butyl)-4-ethynylbenzene, $\mathrm{Pd}\left(\mathrm{PPh}_{3}\right)_{2} \mathrm{Cl}_{2}, \mathrm{CuI}$, toluene/TEA = 2/1, v/v, $55^{\circ} \mathrm{C}, \mathrm{N}_{2}, 24 \mathrm{~h}$; d) 2,3,4,5tetrakis(4-(tert-butyl)phenyl)cyclopenta-2,4-dien-1-one, $\mathrm{Ph}_{2} \mathrm{O}, 260^{\circ} \mathrm{C}, \mathrm{N}_{2}, 36 \mathrm{~h}$; e) DDQ, TfOH, DCM, 0 ' $\mathrm{C}$, $\mathrm{N}_{2}, 2$ h. 

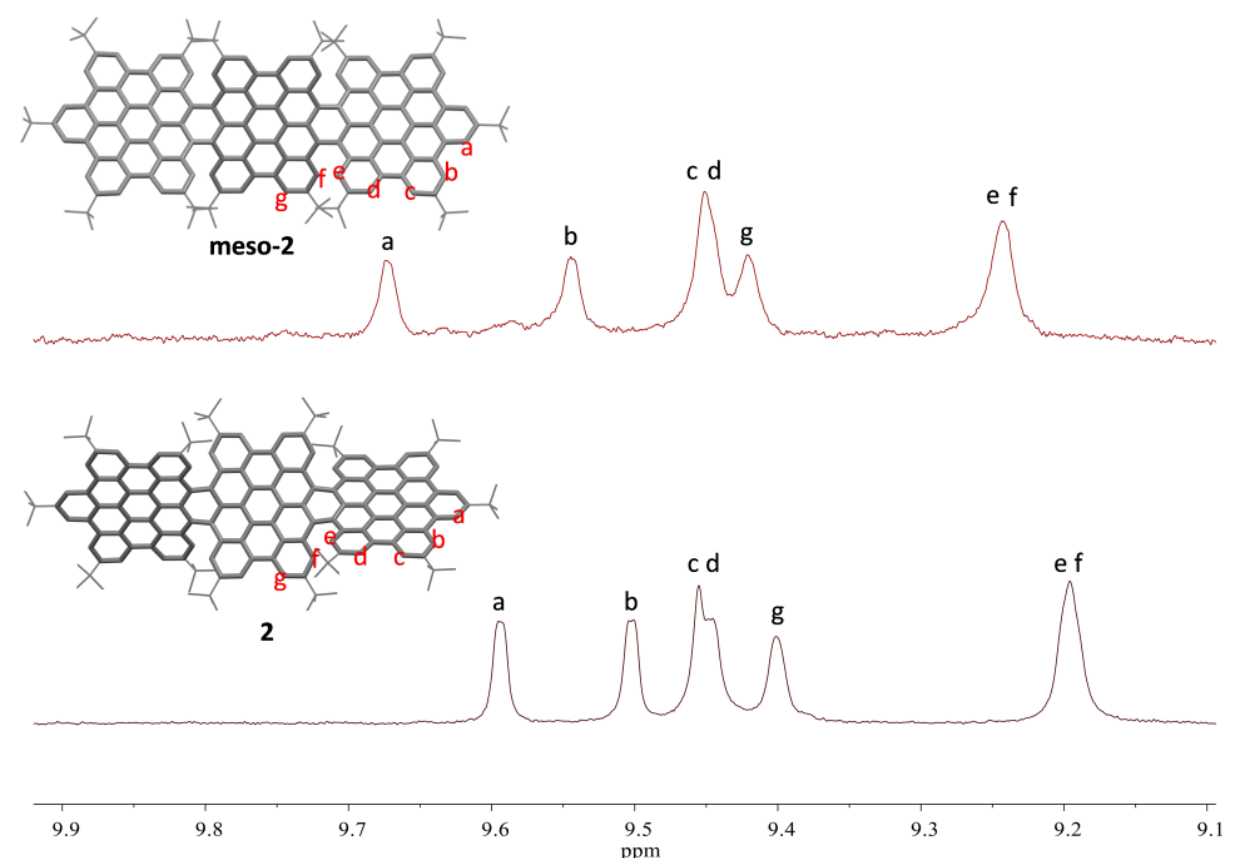

Figure S4. Partial ${ }^{1} \mathrm{H}-\mathrm{NMR}$ spectra of 2 and meso-2 in $\mathrm{CDCl}_{3}$. Assignment of the NMR signals was based on various 2D NMR techniques (data not shown). 


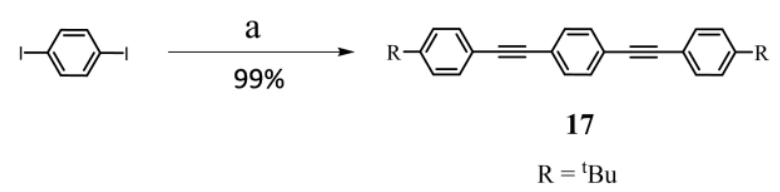

$\mathrm{R}={ }^{\mathrm{t}} \mathrm{Bu}$

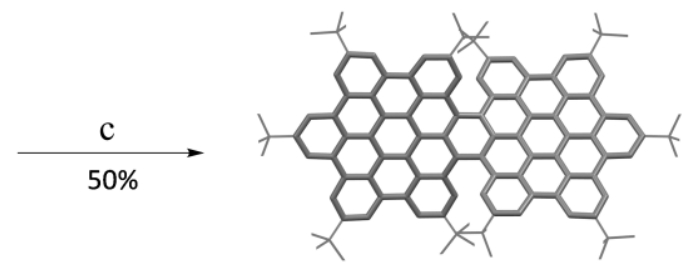

3
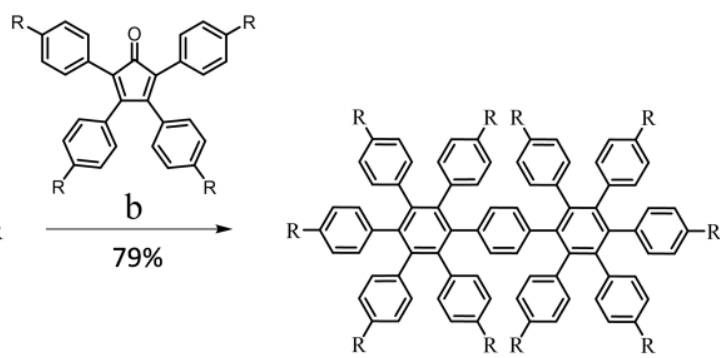

18

Figure S5. Reagents and conditions: a) 1-(tert-butyl)-4-ethynylbenzene, $\mathrm{Pd}\left(\mathrm{PPh}_{3}\right)_{2} \mathrm{Cl}_{2}, \mathrm{CuI}$, toluene/TEA = 4/1, v/v, $55{ }^{\circ} \mathrm{C}, \mathrm{N}_{2}, 12 \mathrm{~h}$; b) 2,3,4,5-tetrakis(4-(tert-butyl)phenyl)cyclopenta-2,4-dien-1-one, $\mathrm{Ph}_{2} \mathrm{O}, 260{ }^{\circ} \mathrm{C}$, $\mathrm{N}_{2}, 4$ d; c) DDQ, TfOH, DCM, $0{ }^{\circ} \mathrm{C}, \mathrm{N}_{2}, 2 \mathrm{~h}$.

17: A mixture of 1,4-diiodobenzene (1.74 g, $5.26 \mathrm{mmol})$, 1-(tert-butyl)-4-ethynylbenzene (2.0 g, $12.64 \mathrm{mmol}), \mathrm{CuI}(40 \mathrm{mg}, 0.21 \mathrm{mmol})$, and $\mathrm{Pd}\left(\mathrm{PPh}_{3}\right)_{2} \mathrm{Cl}_{2}(74 \mathrm{mg}, 0.11 \mathrm{mmol})$ in toluene (40 $\mathrm{mL})$ and triethylamine $(10 \mathrm{~mL})$ was stirred at $55{ }^{\circ} \mathrm{C}$ under a $\mathrm{N}_{2}$ atmosphere for $12 \mathrm{~h}$. After cooling to RT, the solvent was removed under reduced pressure. The product was washed with $\mathrm{MeOH}(100 \mathrm{~mL})$ and dried in air, offering 17 as a white solid $\left(2.02 \mathrm{~g}, 99 \%, \mathrm{~m} . \mathrm{p} .=221-223^{\circ} \mathrm{C}\right)$. ${ }^{1} \mathrm{H}$ NMR $\left(300 \mathrm{MHz}, \mathrm{CDCl}_{3}\right) \delta 7.49(\mathrm{~s}, 4 \mathrm{H}), 7.47(\mathrm{~d}, J=8.6 \mathrm{~Hz}, 4 \mathrm{H}), 7.38(\mathrm{~d}, J=8.6 \mathrm{~Hz}, 4 \mathrm{H})$, $1.33(\mathrm{~s}, 16 \mathrm{H}) .{ }^{13} \mathrm{C} \mathrm{NMR}(75 \mathrm{MHz}, \mathrm{CDCl} 3) \delta 151.9,131.6,125.6,123.3,120.2,91.5,88.7,35.0$ 31.3. MALDI-TOF MS calculated for $\mathrm{C}_{30} \mathrm{H}_{30}, 390.23$; found, $390.30[\mathrm{M}]^{+}$.

18: A mixture of 2,3,4,5-tetrakis(4-(tert-butyl)phenyl)cyclopenta-2,4-dien-1-one (2.0 g, 3.28 $\mathrm{mmol})$ and 17 (320 mg, $0.821 \mathrm{mmol})$ in diphenyl ether $(8 \mathrm{~mL})$ was stirred at $260{ }^{\circ} \mathrm{C}$ under a $\mathrm{N}_{2}$ atmosphere for 4 days. After cooling to RT, $\mathrm{MeOH}(80 \mathrm{~mL})$ was added. The precipitate formed 
was collected and dried in air, offering 18 as a white solid $\left(1.0 \mathrm{~g}, 79 \%\right.$, m.p. > $\left.300{ }^{\circ} \mathrm{C}\right) .{ }^{1} \mathrm{H}$ NMR $\left(300 \mathrm{MHz}, \mathrm{CDCl}_{3}\right) \delta 7.07(\mathrm{~d}, J=7.0 \mathrm{~Hz}, 4 \mathrm{H}), 6.79(\mathrm{~d}, J=8.0 \mathrm{~Hz}, 16 \mathrm{H}), 6.68-6.53(\mathrm{~m}, 16 \mathrm{H})$, $6.45(\mathrm{~d}, J=8.1 \mathrm{~Hz}, 4 \mathrm{H}), 6.27(\mathrm{~s}, 4 \mathrm{H}), 1.24(\mathrm{~s}, 36 \mathrm{H}), 1.11(\mathrm{~s}, 36 \mathrm{H}), 1.10(\mathrm{~s}, 18 \mathrm{H}) .{ }^{13} \mathrm{C} \mathrm{NMR}(75$ $\left.\mathrm{MHz}, \mathrm{CDCl}_{3}\right) \delta 147.6,147.5,147.4,140.9,140.5,140.2,139.4,138.2,138.1,136.9,132.1$, $131.3,131.2,131.0,130.5,123.2,123.1,123.0,34.3,34.2,31.6,31.3$. TOF MS calculated for $\mathrm{C}_{118} \mathrm{H}_{134}, 1552.05$; found, $1552.21[\mathrm{M}]^{+}$.

3: A mixture of DDQ (351 mg, $1.55 \mathrm{mmol})$ and 18 (100 $\mathrm{mg}, 0.064 \mathrm{mmol})$ in dry DCM (100 $\mathrm{mL})$ was stirred at $0{ }^{\circ} \mathrm{C}$ under a $\mathrm{N}_{2}$ atmosphere for $15 \mathrm{~min}$. Then $\mathrm{TfOH}(1 \mathrm{~mL})$ was added. The reaction was stirred at $0{ }^{\circ} \mathrm{C}$ for $2 \mathrm{~h}$. After that, it was quenched with trimethylamine $(2 \mathrm{~mL})$ and washed with water $(100 \mathrm{~mL} \times 3)$. The organic phase was dried over anhydrous $\mathrm{Na}_{2} \mathrm{SO}_{4}$, and concentrated under reduced pressure. The residue was purified by silica gel column chromatography (PE/DCM, v/v, 1/1), offering 3 as a red solid $\left(50 \mathrm{mg}, 50 \%, \mathrm{~m} . \mathrm{p} .>300{ }^{\circ} \mathrm{C}\right) .{ }^{1} \mathrm{H}$ NMR (300 MHz, $\left.\mathrm{CDCl}_{3}\right) \delta 9.46(\mathrm{~s}, 4 \mathrm{H}), 9.41(\mathrm{~d}, 8 \mathrm{H}), 9.36(\mathrm{~s}, 4 \mathrm{H}), 9.16(\mathrm{~s}, 4 \mathrm{H}), 1.90(\mathrm{~s}, 18 \mathrm{H})$, 1.89 (s, 36H), 1.37 (s, 36H). ${ }^{13} \mathrm{C} \mathrm{NMR}\left(75 \mathrm{MHz}, \mathrm{CDCl}_{3}\right) \delta 149.3,148.2,130.9,130.8,130.5$, $127.3,126.5,126.4,124.1,124.0,121.5,120.8,120.7,119.4,119.1,119.0,118.9,118.8,35.8$, 35.2, 32.1, 31.7. MALDI-TOF MS calculated for $\mathrm{C}_{118} \mathrm{H}_{110}, 1527.86$; found, $1528.03[\mathrm{M}]^{+}$. 

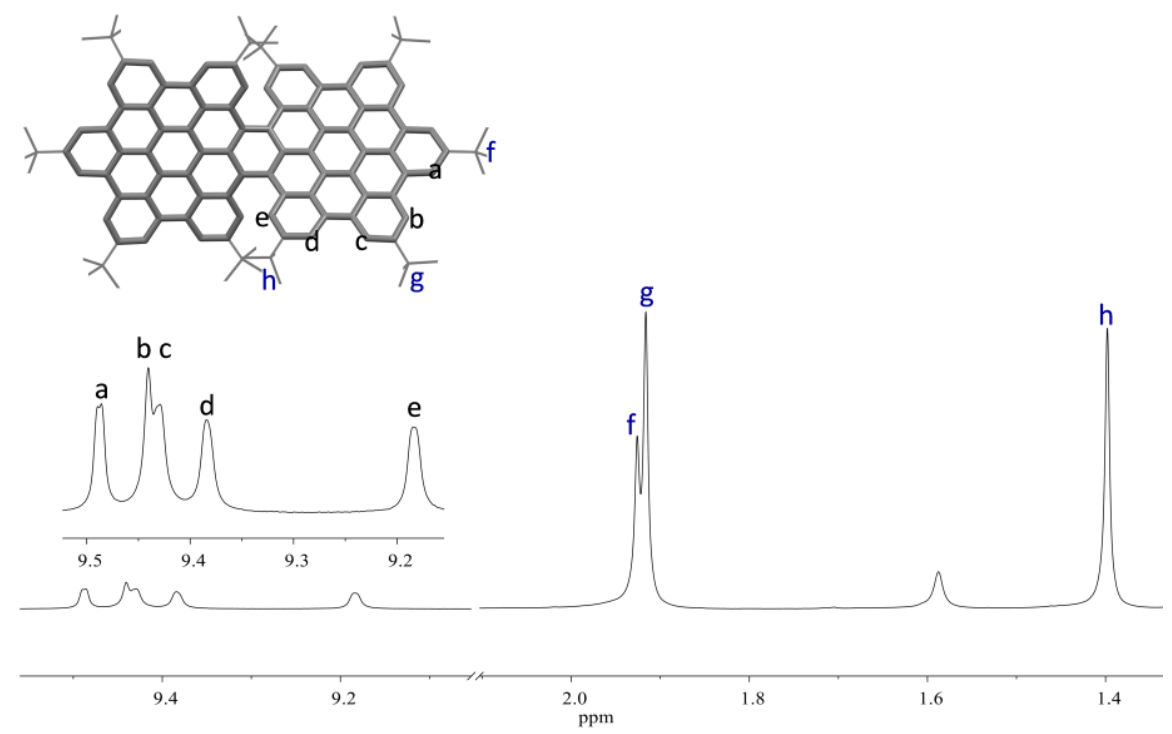

Figure S6. ${ }^{1} \mathrm{H}-\mathrm{NMR}$ spectrum of $\mathbf{3}$ in $\mathrm{CDCl}_{3}$. Assignment of the resonance signals was based on various $2 \mathrm{D}$ NMR techniques. 


\section{X-ray crystallographic studies}

\section{Method for growing single crystals}

Single crystal of 1 was obtained by vapor diffusing of hexane into a solution of $\mathbf{1}(0.9 \mathrm{mg})$ in carbon disulfide $(1.5 \mathrm{~mL}$, containing $100 \mu \mathrm{L}$ of hexane $)$ at $20{ }^{\circ} \mathrm{C}$. Single crystal suitable for Xray structural determination was obtained after three days. The crystal data was collected on a XtaLAB AFC12 (RCD3): Kappa single diffractometer. Note: Addition of hexane (100 $\mu \mathrm{L})$ to carbon disulfide $(1.5 \mathrm{~mL})$ before vapor diffusing is crucial for the successful growth of the single crystals. Otherwise, the carbon disulfide solution will dry quickly without formation of high quality single crystals.

Single crystal of 2 was obtained by slow evaporation of a solution of 2 (1.0 mg) in DCM (2.0 $\mathrm{mL})$ at $0{ }^{\circ} \mathrm{C}$. Single crystal suitable for X-ray structural determination was obtained after three days. The crystal data was collected with the BL17B beamline at Shanghai Synchrotron Radiation Facility.

Single crystal of $\mathbf{3}$ was obtained by slow evaporation of a solution of $\mathbf{3}(1.0 \mathrm{mg})$ in DCM (2.0 $\mathrm{mL}$ ) at $0{ }^{\circ} \mathrm{C}$. A single crystal with sufficient quality for X-ray structural determination was obtained after three days. The crystal data was collected on an Agilent Sapphire3 Gemini Ultra single crystal diffractometer. 
Table S1. Crystallographic data of 1

\begin{tabular}{|c|c|}
\hline & 1 \\
\hline Empirical formula & $\mathrm{C}_{222} \mathrm{H}_{198}$ \\
\hline Formula weight & 2865.79 \\
\hline Temperature/K & 150.0 \\
\hline Crystal system & monoclinic \\
\hline Space group & $P 2{ }_{1} / c$ \\
\hline $\mathrm{a} / \AA ̊ \AA$ & $26.8158(12)$ \\
\hline $\mathrm{b} / \AA ̊ \AA$ & $45.595(3)$ \\
\hline$c / \AA$ & $17.9570(7)$ \\
\hline$\alpha /^{\circ}$ & 90 \\
\hline$\beta /^{\circ}$ & $91.166(4)$ \\
\hline$\gamma /{ }^{\circ}$ & 90 \\
\hline Volume $/ \AA^{3}$ & 21950.6(18) \\
\hline $\mathrm{Z}$ & 4 \\
\hline$\rho c a l c\left(g / \mathrm{cm}^{3}\right)$ & 0.867 \\
\hline$\mu / \mathrm{mm}^{-1}$ & 0.366 \\
\hline $\mathrm{F}(000)$ & 6120.0 \\
\hline Crystal size $/ \mathrm{mm}^{3}$ & 0.350 .230 .11 \\
\hline Radiation & $\mathrm{Cu} \mathrm{K \alpha}(\lambda=1.54184)$ \\
\hline $2 \theta$ range for data collection ${ }^{\circ}$ & 5.088 to 150.21 \\
\hline Index ranges & $-25 \leq \mathrm{h} \leq 24,-34 \leq \mathrm{k} \leq 43,-15 \leq 1 \leq 17$ \\
\hline Reflections collected & 84079 \\
\hline Independent reflections & $19836\left[\mathrm{R}_{\mathrm{int}}=0.0736, \mathrm{R}_{\text {sigma }}=0.0773\right]$ \\
\hline Data/restraints/parameters & $19836 / 313 / 2141$ \\
\hline Goodness-of-fit on $\mathrm{F}^{2}$ & 1.104 \\
\hline Final $R$ indexes $[I>=2 \sigma(I)]$ & $\mathrm{R}_{1}=0.1007, \mathrm{wR}_{2}=0.3016$ \\
\hline Largest diff. peak/hole / e $\AA^{-3}$ & $0.30 /-0.18$ \\
\hline CCDC number & 2019940 \\
\hline
\end{tabular}




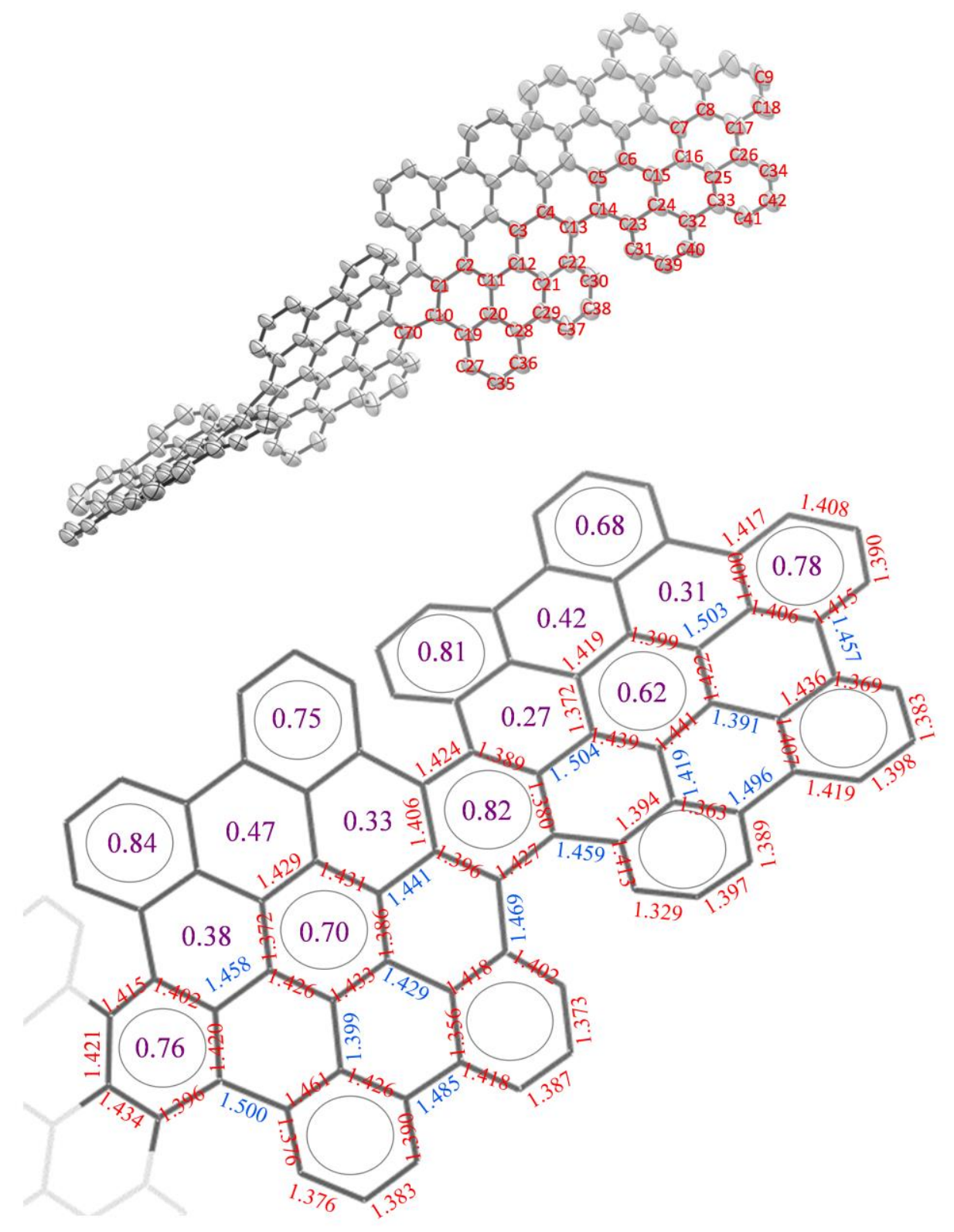

Figure S7. Crystal structure of 1. Representative bond lengths and average HOMA (harmonic oscillator model of aromaticity) values are shown on the Clar structure. Bonds between the Clar rings (labeled in blue) are generally longer than those in the Clar rings (labeled in red). 
Table S2. Representative bond lengths of 1

\begin{tabular}{|c|c|c|}
\hline Atom & Atom & Length/Å \\
\hline $\mathrm{C} 1$ & $\mathrm{C} 2$ & 1.458 \\
\hline $\mathrm{C} 1$ & $\mathrm{C} 10$ & 1.401 \\
\hline $\mathrm{C} 2$ & $\mathrm{C} 11$ & 1.426 \\
\hline $\mathrm{C} 3$ & $\mathrm{C} 4$ & 1.441 \\
\hline $\mathrm{C} 3$ & $\mathrm{C} 12$ & 1.402 \\
\hline $\mathrm{C} 4$ & $\mathrm{C} 13$ & 1.396 \\
\hline $\mathrm{C} 5$ & C6 & 1.504 \\
\hline $\mathrm{C} 5$ & $\mathrm{C} 14$ & 1.380 \\
\hline C6 & $\mathrm{C} 15$ & 1.439 \\
\hline $\mathrm{C} 7$ & $\mathrm{C} 8$ & 1.503 \\
\hline $\mathrm{C} 7$ & $\mathrm{C} 16$ & 1.422 \\
\hline $\mathrm{C} 8$ & $\mathrm{C} 17$ & 1.406 \\
\hline C9 & $\mathrm{C} 18$ & 1.390 \\
\hline $\mathrm{C} 10$ & $\mathrm{C} 19$ & 1.500 \\
\hline $\mathrm{C} 10$ & $\mathrm{C} 70$ & 1.396 \\
\hline $\mathrm{C} 11$ & $\mathrm{C} 12$ & 1.433 \\
\hline $\mathrm{C} 11$ & $\mathrm{C} 20$ & 1.399 \\
\hline $\mathrm{C} 12$ & $\mathrm{C} 21$ & 1.429 \\
\hline $\mathrm{C} 13$ & $\mathrm{C} 14$ & 1.427 \\
\hline $\mathrm{C} 13$ & $\mathrm{C} 22$ & 1.469 \\
\hline $\mathrm{C} 14$ & $\mathrm{C} 23$ & 1.459 \\
\hline $\mathrm{C} 15$ & $\mathrm{C} 16$ & 1.441 \\
\hline $\mathrm{C} 15$ & $\mathrm{C} 24$ & 1.419 \\
\hline $\mathrm{C} 16$ & $\mathrm{C} 25$ & 1.391 \\
\hline $\mathrm{C} 17$ & $\mathrm{C} 18$ & 1.415 \\
\hline $\mathrm{C} 17$ & $\mathrm{C} 26$ & 1.457 \\
\hline $\mathrm{C} 19$ & $\mathrm{C} 20$ & 1.461 \\
\hline $\mathrm{C} 19$ & $\mathrm{C} 27$ & 1.385 \\
\hline $\mathrm{C} 20$ & $\mathrm{C} 28$ & 1.426 \\
\hline $\mathrm{C} 21$ & $\mathrm{C} 22$ & 1.418 \\
\hline $\mathrm{C} 21$ & $\mathrm{C} 29$ & 1.371 \\
\hline $\mathrm{C} 22$ & $\mathrm{C} 30$ & 1.402 \\
\hline $\mathrm{C} 23$ & $\mathrm{C} 24$ & 1.394 \\
\hline $\mathrm{C} 23$ & $\mathrm{C} 31$ & 1.400 \\
\hline $\mathrm{C} 24$ & $\mathrm{C} 32$ & 1.363 \\
\hline $\mathrm{C} 25$ & $\mathrm{C} 26$ & 1.436 \\
\hline $\mathrm{C} 25$ & $\mathrm{C} 33$ & 1.415 \\
\hline
\end{tabular}




\begin{tabular}{lll}
$\mathrm{C} 26$ & $\mathrm{C} 34$ & 1.369 \\
$\mathrm{C} 27$ & $\mathrm{C} 35$ & 1.376 \\
$\mathrm{C} 28$ & $\mathrm{C} 29$ & 1.485 \\
$\mathrm{C} 28$ & $\mathrm{C} 36$ & 1.390 \\
$\mathrm{C} 29$ & $\mathrm{C} 37$ & 1.418 \\
$\mathrm{C} 30$ & $\mathrm{C} 38$ & 1.373 \\
$\mathrm{C} 31$ & $\mathrm{C} 39$ & 1.329 \\
$\mathrm{C} 32$ & $\mathrm{C} 33$ & 1.496 \\
$\mathrm{C} 32$ & $\mathrm{C} 40$ & 1.389 \\
$\mathrm{C} 33$ & $\mathrm{C} 41$ & 1.419 \\
$\mathrm{C} 34$ & $\mathrm{C} 42$ & 1.383 \\
$\mathrm{C} 35$ & $\mathrm{C} 36$ & 1.383 \\
$\mathrm{C} 37$ & $\mathrm{C} 38$ & 1.387 \\
$\mathrm{C} 39$ & $\mathrm{C} 40$ & 1.397 \\
$\mathrm{C} 41$ & $\mathrm{C} 42$ & 1.398 \\
\hline
\end{tabular}


Table S3. Crystallographic data of 2

\begin{tabular}{|c|c|}
\hline & 2 \\
\hline Empirical formula & $\mathrm{C}_{170} \mathrm{H}_{154}$ \\
\hline Formula weight & 2196.92 \\
\hline Temperature/K & 293(2) \\
\hline Crystal system & orthorhombic \\
\hline Space group & Pbcn \\
\hline $\mathrm{a} / \AA ̊$ & $43.735(9)$ \\
\hline $\mathrm{b} / \AA$ & 21.613(4) \\
\hline $\mathrm{c} / \AA ̊$ & $16.915(3)$ \\
\hline$\alpha /{ }^{\circ}$ & 90 \\
\hline$\beta /^{\circ}$ & 90 \\
\hline$\gamma /{ }^{\circ}$ & 90 \\
\hline Volume $/ \AA^{3}$ & $15989(6)$ \\
\hline $\mathrm{Z}$ & 4 \\
\hline$\rho_{\text {calc }} \mathrm{g} / \mathrm{cm}^{3}$ & 0.913 \\
\hline$\mu / \mathrm{mm}^{-1}$ & 0.051 \\
\hline $\mathrm{F}(000)$ & 4696.0 \\
\hline Crystal size $/ \mathrm{mm}^{3}$ & $0.25 \times 0.16 \times 0.10$ \\
\hline Radiation & $\operatorname{MoK} \alpha(\lambda=0.71073)$ \\
\hline $2 \theta$ range for data collection $/^{\circ}$ & 3.196 to 54.372 \\
\hline Index ranges & $-56 \leq \mathrm{h} \leq 56,-27 \leq \mathrm{k} \leq 27,0 \leq 1 \leq 21$ \\
\hline Reflections collected & 62808 \\
\hline Independent reflections & $17703\left[R_{\text {int }}=0.0783, R_{\text {sigma }}=0.0582\right]$ \\
\hline Data/restraints/parameters & $17703 / 33 / 828$ \\
\hline Goodness-of-fit on $\mathrm{F}^{2}$ & 1.040 \\
\hline Final $R$ indexes $[I>=2 \sigma(I)]$ & $\mathrm{R}_{1}=0.0945, \mathrm{wR}_{2}=0.2882$ \\
\hline Largest diff. peak/hole / e $\AA^{-3}$ & $0.47 /-0.35$ \\
\hline CCDC Number & 2010141 \\
\hline
\end{tabular}




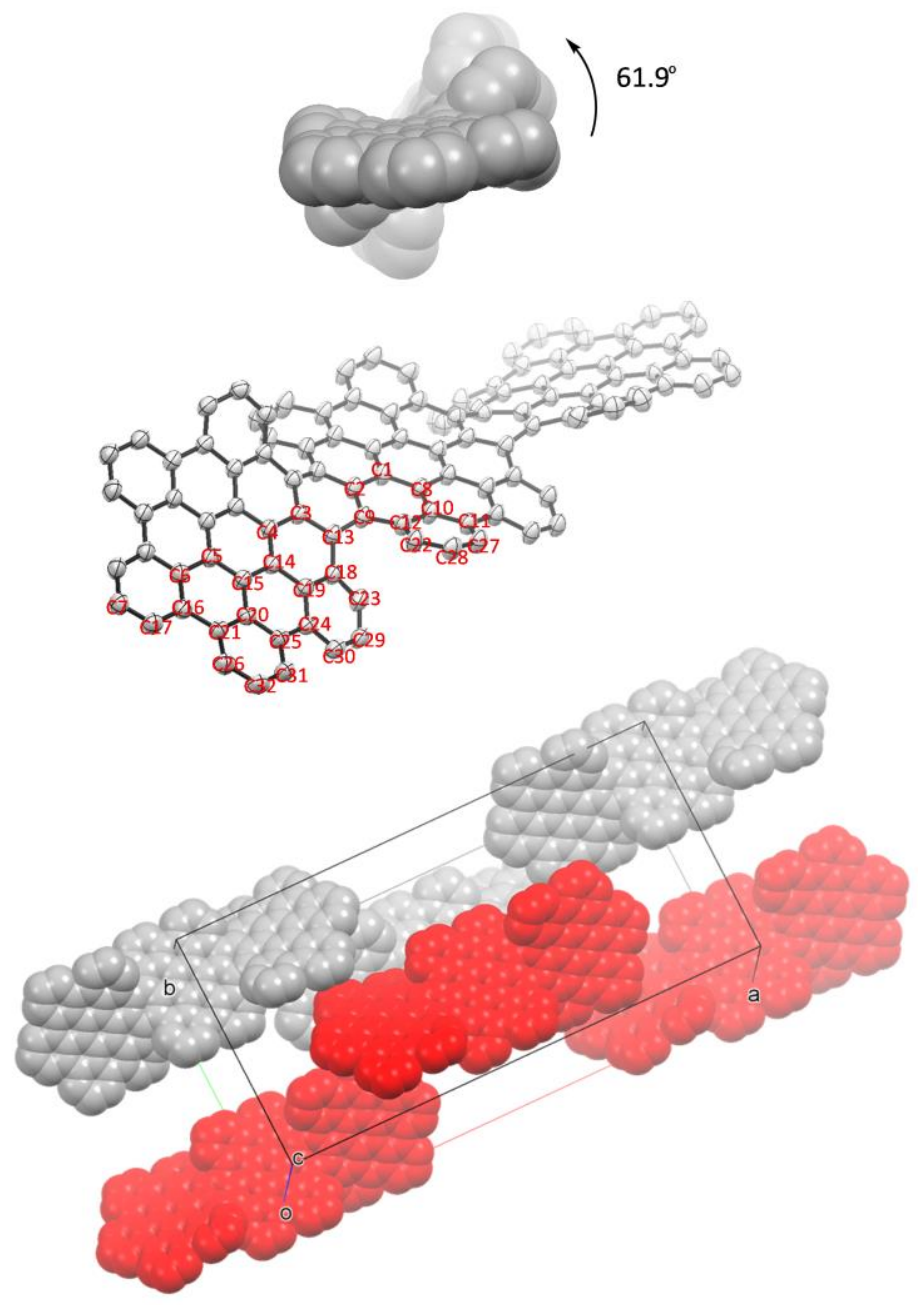

Figure S8. Crystal structure of 2 with an end-to-end twist of 61.9. Thermal ellipsoids are shown with 50\% probability. In the unit cell (bottom), both enantiomers of $\mathbf{2}$ are present. The $(P)$ - and $(M)$-form of 2 were shown in red and gray, respectively. They are deposited in the Pbcn space group via van de Waals contacts without $\pi-\pi$ interactions. The t-butyl groups are omitted for clarity.

Table S4. Representative bond lengths of 2

\begin{tabular}{ccc}
\hline Atom & Atom & Length/̊̊ \\
\hline $\mathrm{C} 1$ & $\mathrm{C} 2$ & $1.446(3)$ \\
$\mathrm{C} 1$ & $\mathrm{C} 8$ & $1.425(3)$ \\
$\mathrm{C} 2$ & $\mathrm{C} 9$ & $1.416(3)$ \\
$\mathrm{C} 3$ & $\mathrm{C} 4$ & $1.436(3)$ \\
$\mathrm{C} 3$ & $\mathrm{C} 13$ & $1.426(3)$ \\
\hline
\end{tabular}




\begin{tabular}{|c|c|c|}
\hline $\mathrm{C} 4$ & $\mathrm{C} 14$ & $1.419(3)$ \\
\hline C5 & $\mathrm{C} 6$ & $1.442(3)$ \\
\hline $\mathrm{C} 5$ & $\mathrm{C} 15$ & $1.428(3)$ \\
\hline C6 & $\mathrm{C} 16$ & $1.424(3)$ \\
\hline $\mathrm{C} 7$ & $\mathrm{C} 17$ & $1.385(4)$ \\
\hline $\mathrm{C} 7$ & C55 & $1.397(4)$ \\
\hline $\mathrm{C} 7$ & $\mathrm{C} 82$ & $1.524(4)$ \\
\hline $\mathrm{C} 8$ & $\mathrm{C} 10$ & $1.441(3)$ \\
\hline $\mathrm{C} 9$ & $\mathrm{C} 12$ & $1.462(3)$ \\
\hline $\mathrm{C} 9$ & $\mathrm{C} 13$ & $1.421(3)$ \\
\hline $\mathrm{C} 10$ & $\mathrm{C} 11$ & $1.428(3)$ \\
\hline $\mathrm{C} 10$ & $\mathrm{C} 12$ & $1.409(4)$ \\
\hline $\mathrm{C} 11$ & $\mathrm{C} 27$ & $1.394(4)$ \\
\hline $\mathrm{C} 12$ & $\mathrm{C} 22$ & $1.395(3)$ \\
\hline $\mathrm{C} 13$ & $\mathrm{C} 18$ & $1.456(3)$ \\
\hline $\mathrm{C} 14$ & $\mathrm{C} 15$ & $1.400(3)$ \\
\hline C14 & $\mathrm{C} 19$ & $1.444(3)$ \\
\hline $\mathrm{C} 15$ & $\mathrm{C} 20$ & $1.440(3)$ \\
\hline $\mathrm{C} 16$ & $\mathrm{C} 17$ & $1.396(4)$ \\
\hline C16 & $\mathrm{C} 21$ & $1.465(4)$ \\
\hline $\mathrm{C} 18$ & $\mathrm{C} 19$ & $1.412(3)$ \\
\hline $\mathrm{C} 18$ & $\mathrm{C} 23$ & $1.406(4)$ \\
\hline C19 & $\mathrm{C} 24$ & $1.414(3)$ \\
\hline $\mathrm{C} 20$ & $\mathrm{C} 21$ & $1.411(4)$ \\
\hline $\mathrm{C} 20$ & $\mathrm{C} 25$ & $1.421(4)$ \\
\hline $\mathrm{C} 21$ & $\mathrm{C} 26$ & $1.397(4)$ \\
\hline $\mathrm{C} 22$ & $\mathrm{C} 28$ & $1.382(4)$ \\
\hline $\mathrm{C} 23$ & $\mathrm{C} 29$ & $1.382(4)$ \\
\hline $\mathrm{C} 24$ & $\mathrm{C} 25$ & $1.465(3)$ \\
\hline $\mathrm{C} 24$ & $\mathrm{C} 30$ & $1.369(4)$ \\
\hline $\mathrm{C} 25$ & $\mathrm{C} 31$ & $1.397(3)$ \\
\hline $\mathrm{C} 26$ & $\mathrm{C} 32$ & $1.382(4)$ \\
\hline $\mathrm{C} 27$ & $\mathrm{C} 28$ & $1.373(4)$ \\
\hline $\mathrm{C} 29$ & $\mathrm{C} 30$ & $1.416(4)$ \\
\hline C31 & $\mathrm{C} 32$ & $1.385(4)$ \\
\hline
\end{tabular}




\section{Crystallographic data of 3}

The quality of the single crystal of $\mathbf{3}$ was low. Therefore, further refinement of the X-ray structure was not possible. Despite this, key structural features of $\mathbf{3}$ in the solid state are still available, including the twist angle as well as the continuous intermolecular $\pi$ - $\pi$ stacking (blue arrow).
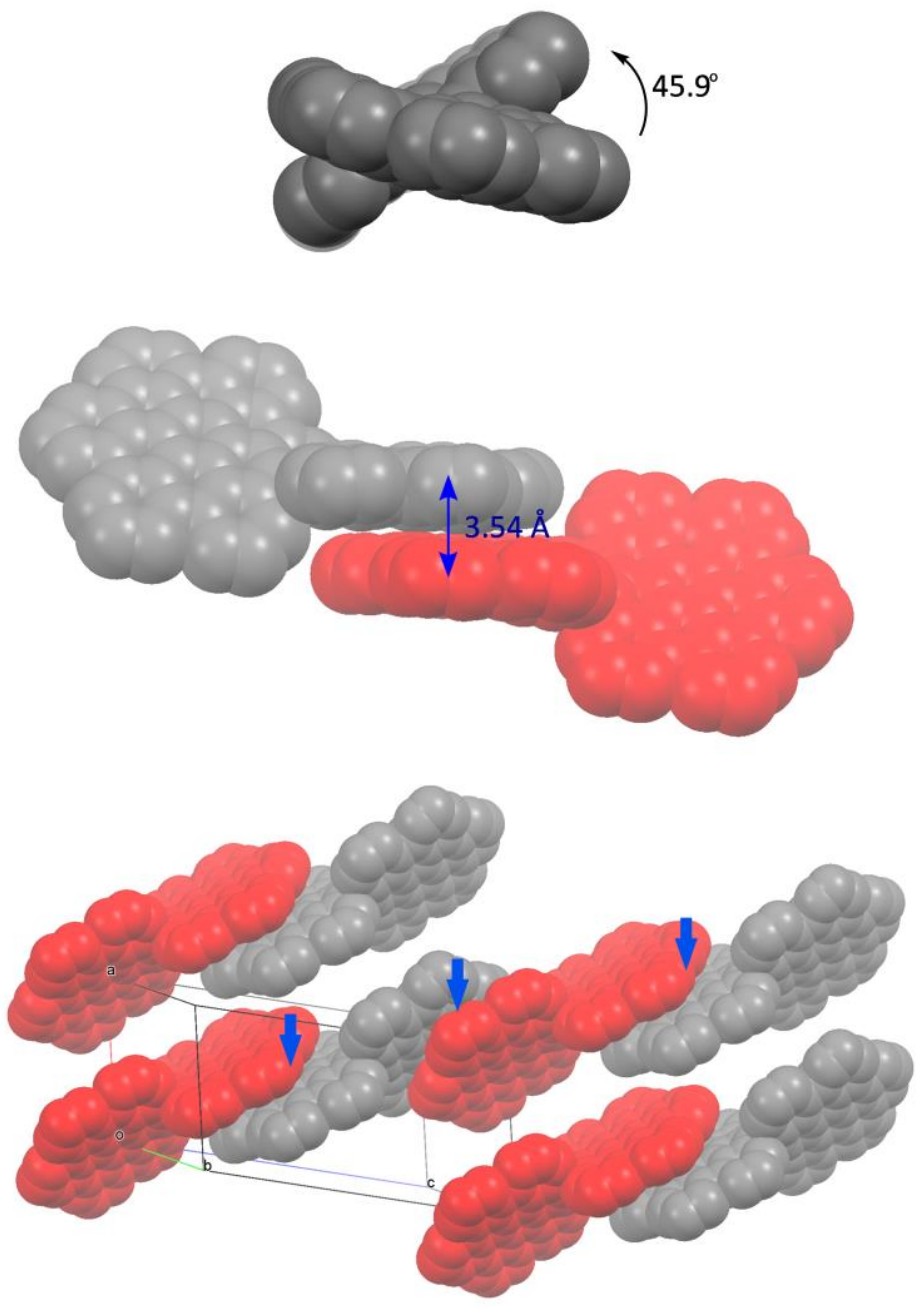

Figure S9. Single crystal X-ray structure of $\mathbf{3}$. The $(P)$ - and $(M)$-form of $\mathbf{3}$ were shown in red and gray, respectively. $\mathbf{3}$ was deposited in the space group of $P \overline{1}$. The t-butyl groups are omitted for clarity. 
4. Optical properties and DFT calculations
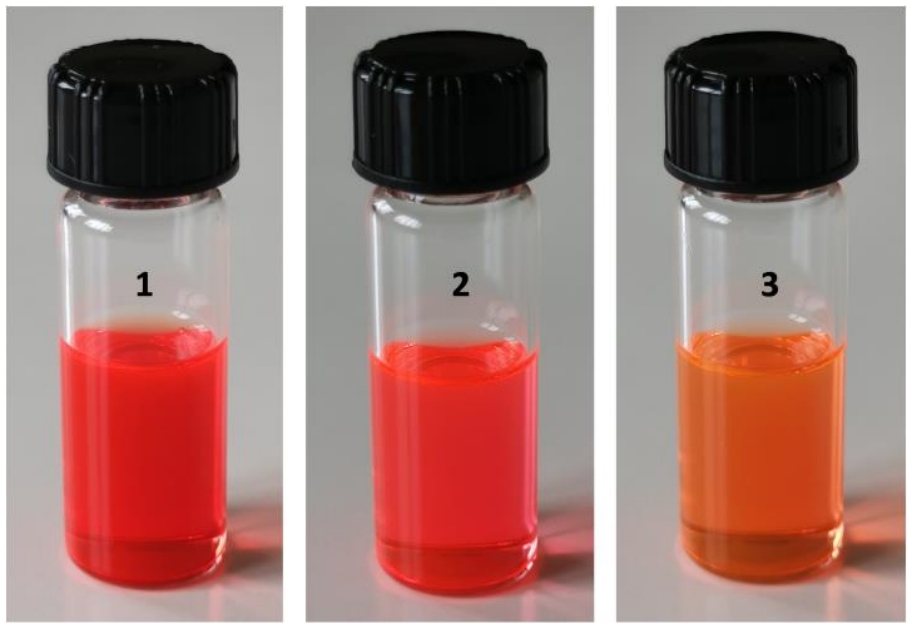

Figure S10. Visualized images of 1-3 dissolved in DCM. 


\section{DFT calculations}

Gaussian 16 software packages was used for the theoretical studies. ${ }^{1}$ The density functional theory (DFT) at the level of B3LYP/6-31G(d) was used for geometry optimization. The same base set was employed for the time-dependent (TD) DFT calculation.

\section{DFT and TD-DFT results of 1}
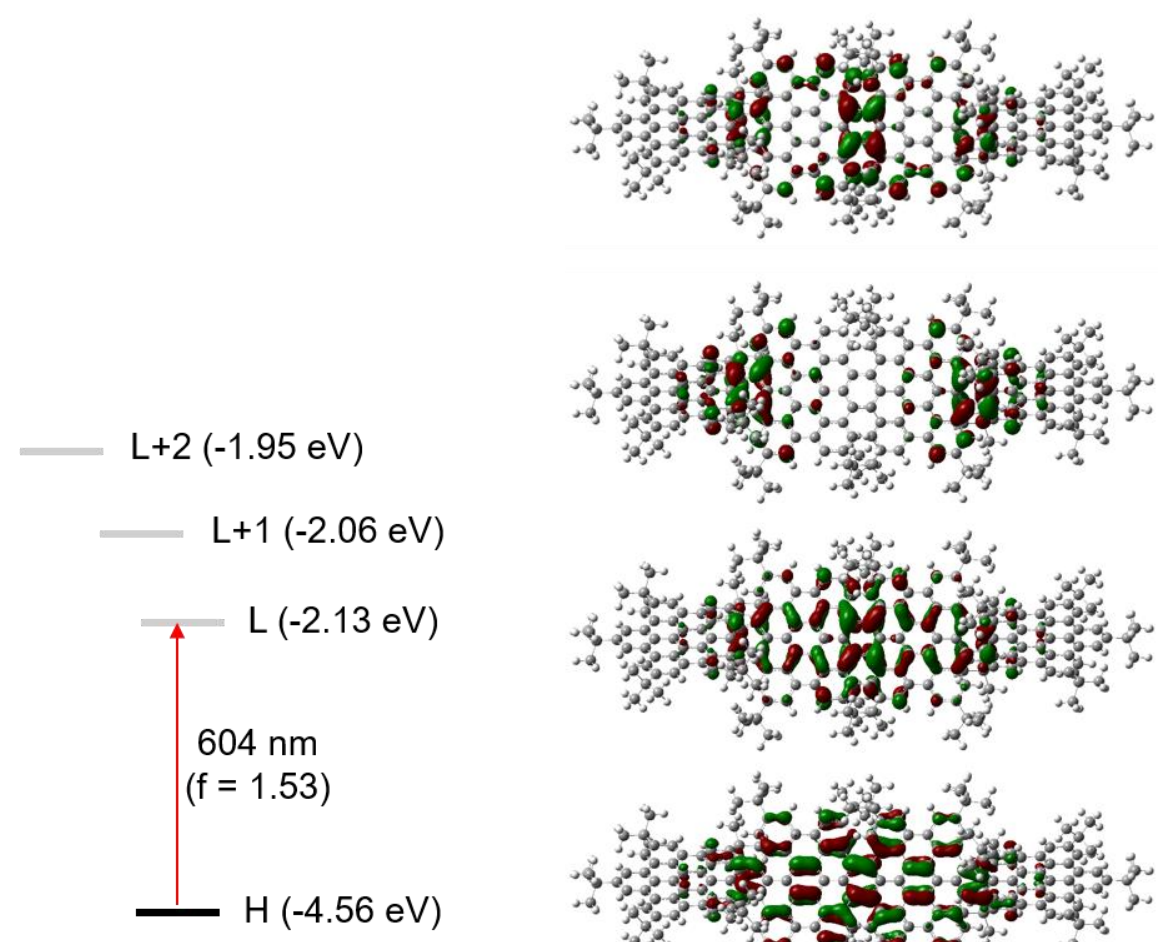

$-\mathrm{H}-1(-4.69 \mathrm{eV})$
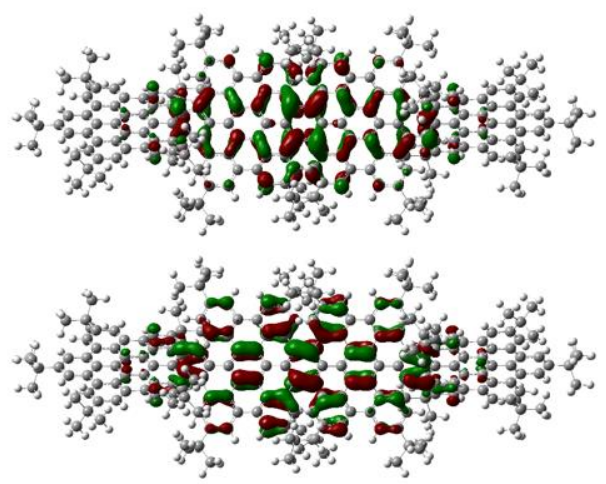

$-\mathrm{H}-2(-4.82 \mathrm{eV})$

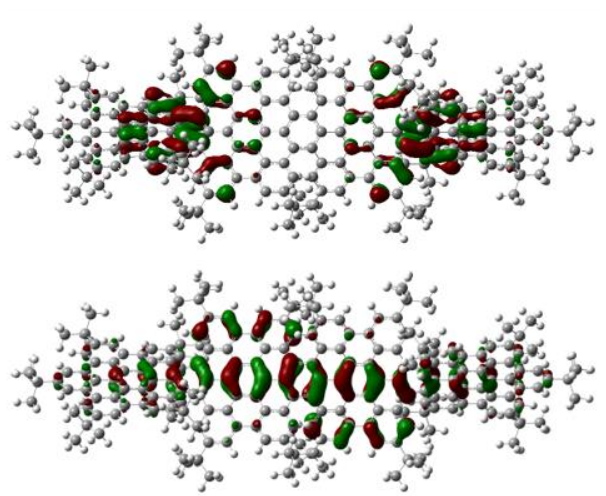

Figure S11. Kohn-Sham molecular orbitals and their energies of 1 . TD-DFT result for the $\mathrm{S}_{0}-\mathrm{S}_{1}$ transition is indicated with a red arrow. 

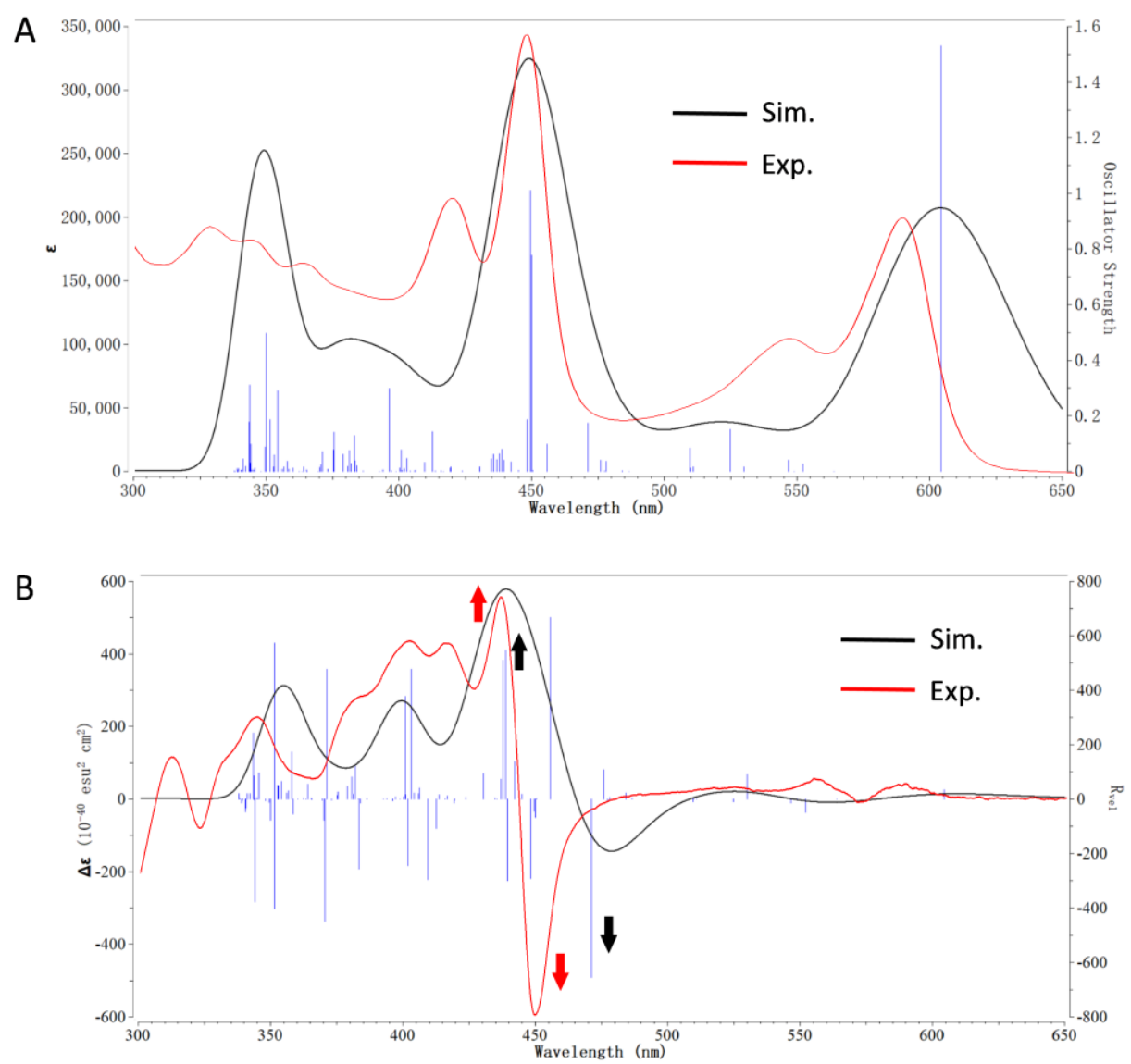

Figure S12. Computational and experimental UV-Vis absorption (A) and CD (B) spectra of $(P)-\mathbf{1}$. The arrows show the sign of the corresponding $\mathrm{CD}$ signals. Because of the weak experimental and computational CD signals at longer wavelength, it is difficult to compare the CD pattern in the spectral range above $500 \mathrm{~nm}$.

Table S5. TD-DFT data of 1

\begin{tabular}{|c|c|c|c|c|}
\hline Transition & $\begin{array}{c}\text { Energy } \\
(\mathrm{eV})\end{array}$ & $\begin{array}{l}\text { Wavelength } \\
(\mathrm{nm})\end{array}$ & $\begin{array}{l}\text { Oscillator } \\
\text { strength }\end{array}$ & Contribution of Orbitals \\
\hline $\mathrm{S}_{0} \rightarrow \mathrm{S}_{1}$ & 2.0517 & 604.31 & 1.530 & $\begin{array}{r}\mathrm{HOMO}-1 \rightarrow \mathrm{LUMO}+1(7 \%) \\
\mathrm{HOMO} \rightarrow \text { LUMO }(91 \%)\end{array}$ \\
\hline $\mathrm{S}_{0} \rightarrow \mathrm{S}_{2}$ & 2.1988 & 563.87 & 0.000 & $\begin{array}{l}\text { HOMO }-1 \rightarrow \text { LUMO }(22 \%) \\
\text { HOMO } \rightarrow \text { LUMO+1 }(73 \%)\end{array}$ \\
\hline $\mathrm{S}_{0} \rightarrow \mathrm{S}_{3}$ & 2.2455 & 552.16 & 0.027 & $\begin{array}{l}\text { HOMO }-2 \rightarrow \text { LUMO }(56 \%) \\
\text { HOMO } \rightarrow \text { LUMO+2 }(10 \%) \\
\text { HOMO } \rightarrow \text { LUMO+3 }(20 \%)\end{array}$ \\
\hline $\mathrm{S}_{0} \rightarrow \mathrm{S}_{4}$ & 2.2582 & 549.03 & 0.000 & $\begin{array}{r}\text { HOMO- } 1 \rightarrow \text { LUMO }(70 \%) \\
\text { HOMO- } 1 \rightarrow \text { LUMO+2 (7\%) } \\
\text { HOMO } \rightarrow \text { LUMO+1 }(17 \%)\end{array}$ \\
\hline
\end{tabular}




\begin{tabular}{|c|c|c|c|c|}
\hline $\mathrm{S}_{0} \rightarrow \mathrm{S}_{5}$ & 2.2679 & 546.70 & 0.042 & $\begin{array}{r}\mathrm{HOMO}-1 \rightarrow \mathrm{LUMO}+1(7 \%) \\
\mathrm{HOMO} \rightarrow \mathrm{LUMO}+2(78 \%)\end{array}$ \\
\hline \multirow{4}{*}{$\mathrm{S}_{0} \rightarrow \mathrm{S}_{6}$} & \multirow{4}{*}{2.3388} & \multirow{4}{*}{530.13} & \multirow{4}{*}{0.016} & HOMO-4 $\rightarrow$ LUMO (19\%) \\
\hline & & & & HOMO-2 $\rightarrow$ LUMO+1 (46\%) \\
\hline & & & & HOMO- $1 \rightarrow$ LUMO+3 (14\%) \\
\hline & & & & $\mathrm{HOMO} \rightarrow \mathrm{LUMO}+4(8 \%)$ \\
\hline \multirow{2}{*}{$\mathrm{S}_{0} \rightarrow \mathrm{S}_{7}$} & \multirow{2}{*}{2.3624} & \multirow{2}{*}{524.83} & \multirow{2}{*}{0.151} & HOMO-3 $\rightarrow$ LUMO $(41 \%)$ \\
\hline & & & & HOMO- $1 \rightarrow$ LUMO+1 $(48 \%)$ \\
\hline \multirow{4}{*}{$\mathrm{S}_{0} \rightarrow \mathrm{S}_{8}$} & \multirow{4}{*}{2.4268} & \multirow{4}{*}{510.89} & \multirow{4}{*}{0.017} & HOMO-4 $\rightarrow$ LUMO+1 (14\%) \\
\hline & & & & HOMO-3 $\rightarrow$ LUMO $(8 \%)$ \\
\hline & & & & HOMO-3 $\rightarrow$ LUMO $+3(6 \%)$ \\
\hline & & & & $\mathrm{HOMO}-2 \rightarrow \mathrm{LUMO}+2(49 \%)$ \\
\hline \multirow{3}{*}{$\mathrm{S}_{0} \rightarrow \mathrm{S}_{9}$} & \multirow{3}{*}{2.4312} & \multirow{3}{*}{509.97} & \multirow{3}{*}{0.012} & HOMO-3 $\rightarrow$ LUMO (6\%) \\
\hline & & & & $\mathrm{HOMO}-3 \rightarrow \mathrm{LUMO}+1(17 \%)$ \\
\hline & & & & HOMO- $1 \rightarrow$ LUMO $+2(60 \%)$ \\
\hline \multirow{4}{*}{$\mathrm{S}_{0} \rightarrow \mathrm{S}_{10}$} & \multirow{4}{*}{2.4331} & \multirow{4}{*}{509.56} & \multirow{4}{*}{0.084} & HOMO-3 $\rightarrow$ LUMO $(34 \%)$ \\
\hline & & & & $\mathrm{HOMO}-3 \rightarrow \mathrm{LUMO}+2(12 \%)$ \\
\hline & & & & HOMO- $1 \rightarrow$ LUMO+1 $(17 \%)$ \\
\hline & & & & $\mathrm{HOMO}-1 \rightarrow \mathrm{LUMO}+2(11 \%)$ \\
\hline \multirow{5}{*}{$\mathrm{S}_{0} \rightarrow \mathrm{S}_{11}$} & \multirow{5}{*}{2.5479} & \multirow{5}{*}{486.61} & \multirow{5}{*}{0.000} & HOMO-4 $\rightarrow$ LUMO (6\%) \\
\hline & & & & HOMO-3 $\rightarrow$ LUMO+1 (55\%) \\
\hline & & & & HOMO-2 $\rightarrow$ LUMO+1 (10\%) \\
\hline & & & & HOMO- $1 \rightarrow$ LUMO $(6 \%)$ \\
\hline & & & & HOMO- $1 \rightarrow$ LUMO $+2(16 \%)$ \\
\hline \multirow{5}{*}{$\mathrm{S}_{0} \rightarrow \mathrm{S}_{12}$} & \multirow{5}{*}{2.5608} & \multirow{5}{*}{484.16} & \multirow{5}{*}{0.003} & HOMO-6 $\rightarrow$ LUMO $(6 \%)$ \\
\hline & & & & HOMO-4 $\rightarrow$ LUMO (47\%) \\
\hline & & & & HOMO-4 $\rightarrow$ LUMO+2 (6\%) \\
\hline & & & & $\mathrm{HOMO}-3 \rightarrow \mathrm{LUMO}+1(13 \%)$ \\
\hline & & & & HOMO-2 $\rightarrow$ LUMO+1 (16\%) \\
\hline \multirow{3}{*}{$\mathrm{S}_{0} \rightarrow \mathrm{S}_{13}$} & \multirow{3}{*}{2.5932} & & & $\mathrm{HOMO}-3 \rightarrow \mathrm{LUMO}+2(57 \%)$ \\
\hline & & 478.12 & 0.038 & $\mathrm{HOMO}-2 \rightarrow \mathrm{LUMO}+2(14 \%)$ \\
\hline & & & & HOMO- $1 \rightarrow$ LUMO+1 (9\%) \\
\hline & & & & HOMO-6 $\rightarrow$ LUMO $(35 \%)$ \\
\hline $\mathrm{S}_{0} \rightarrow \mathrm{S}_{14}$ & 2.5971 & 477.40 & 0.003 & HOMO-5 $\rightarrow$ LUMO+1 (28\%) \\
\hline & & & & $\mathrm{HOMO}-4 \rightarrow \mathrm{LUMO}+2(14 \%)$ \\
\hline
\end{tabular}


HOMO-6 $\rightarrow$ LUMO+1 (8\%)

HOMO-5 $\rightarrow$ LUMO (23\%)

$\mathrm{S}_{0} \rightarrow \mathrm{S}_{15}$

2.6053

475.89

0.041

HOMO-5 $\rightarrow$ LUMO+2 (9\%)

HOMO-4 $\rightarrow$ LUMO+1 (14\%)

HOMO-3 $\rightarrow$ LUMO+2 (19\%)

HOMO- $2 \rightarrow$ LUMO+2 (12\%)

\begin{tabular}{|c|c|c|c|c|}
\hline \multirow{3}{*}{$\mathrm{S}_{0} \rightarrow \mathrm{S}_{16}$} & \multirow{3}{*}{2.6316} & \multirow{3}{*}{471.14} & \multirow{3}{*}{0.175} & HOMO-5 $\rightarrow$ LUMO (13\%) \\
\hline & & & & HOMO-2 $\rightarrow$ LUMO $(29 \%)$ \\
\hline & & & & $\mathrm{HOMO} \rightarrow \mathrm{LUMO}+3(45 \%)$ \\
\hline \multirow{6}{*}{$\mathrm{S}_{0} \rightarrow \mathrm{S}_{17}$} & \multirow{6}{*}{2.7204} & \multirow{6}{*}{455.75} & \multirow{6}{*}{0.098} & HOMO-6 $\rightarrow$ LUMO $(9 \%)$ \\
\hline & & & & HOMO-4 $\rightarrow$ LUMO (8\%) \\
\hline & & & & $\mathrm{HOMO}-4 \rightarrow \mathrm{LUMO}+2(19 \%)$ \\
\hline & & & & HOMO-2 $\rightarrow$ LUMO+1 (18\%) \\
\hline & & & & HOMO- $1 \rightarrow$ LUMO+3 (31\%) \\
\hline & & & & $\mathrm{HOMO} \rightarrow \mathrm{LUMO}+4(6 \%)$ \\
\hline \multirow{3}{*}{$\mathrm{S}_{0} \rightarrow \mathrm{S}_{18}$} & \multirow{3}{*}{2.7532} & \multirow{3}{*}{450.33} & \multirow{3}{*}{0.004} & HOMO-7 $\rightarrow$ LUMO (45\%) \\
\hline & & & & $\mathrm{HOMO}-7 \rightarrow \mathrm{LUMO}+1(31 \%)$ \\
\hline & & & & $\mathrm{HOMO}-7 \rightarrow \mathrm{LUMO}+2(7 \%)$ \\
\hline \multirow{4}{*}{$\mathrm{S}_{0} \rightarrow \mathrm{S}_{19}$} & \multirow{4}{*}{2.7546} & \multirow{4}{*}{450.10} & \multirow{4}{*}{0.777} & HOMO-7 $\rightarrow$ LUMO $(26 \%)$ \\
\hline & & & & $\mathrm{HOMO}-7 \rightarrow \mathrm{LUMO}+2(6 \%)$ \\
\hline & & & & HOMO-7 $\rightarrow$ LUMO+1 (22\%) \\
\hline & & & & $\mathrm{HOMO}-2 \rightarrow \mathrm{LUMO}+3(33 \%)$ \\
\hline \multirow{3}{*}{$\mathrm{S}_{0} \rightarrow \mathrm{S}_{20}$} & \multirow{3}{*}{2.7574} & \multirow{3}{*}{449.63} & \multirow{3}{*}{1.010} & HOMO-7 $\rightarrow$ LUMO $(15 \%)$ \\
\hline & & & & HOMO-7 $\rightarrow$ LUMO+1 (11\%) \\
\hline & & & & $\mathrm{HOMO}-2 \rightarrow \mathrm{LUMO}+3(53 \%)$ \\
\hline \multirow{5}{*}{$\mathrm{S}_{0} \rightarrow \mathrm{S}_{21}$} & \multirow{5}{*}{2.7654} & \multirow{5}{*}{448.34} & \multirow{5}{*}{0.187} & HOMO-6 $\rightarrow$ LUMO+1 $(12 \%)$ \\
\hline & & & & HOMO-5 $\rightarrow$ LUMO $(10 \%)$ \\
\hline & & & & $\mathrm{HOMO}-4 \rightarrow \mathrm{LUMO}+1(40 \%)$ \\
\hline & & & & $\mathrm{HOMO}-2 \rightarrow \mathrm{LUMO}+2(6 \%)$ \\
\hline & & & & $\mathrm{HOMO} \rightarrow \mathrm{LUMO}+3(21 \%)$ \\
\hline
\end{tabular}




\begin{tabular}{|c|c|c|c|c|}
\hline \multirow{5}{*}{$\mathrm{S}_{0} \rightarrow \mathrm{S}_{22}$} & \multirow{5}{*}{2.7860} & \multirow{5}{*}{445.03} & \multirow{5}{*}{0.003} & $\mathrm{HOMO}-6 \rightarrow \mathrm{LUMO}+2(9 \%)$ \\
\hline & & & & HOMO-5 $\rightarrow$ LUMO+1 (7\%) \\
\hline & & & & HOMO-4 $\rightarrow$ LUMO (6\%) \\
\hline & & & & HOMO- $1 \rightarrow$ LUMO+3 (20\%) \\
\hline & & & & $\mathrm{HOMO} \rightarrow \mathrm{LUMO}+4(41 \%)$ \\
\hline \multirow{5}{*}{$\mathrm{S}_{0} \rightarrow \mathrm{S}_{23}$} & \multirow{5}{*}{2.8036} & \multirow{5}{*}{442.23} & \multirow{5}{*}{0.035} & HOMO-6 $\rightarrow$ LUMO+2 (13\%) \\
\hline & & & & HOMO-5 $\rightarrow$ LUMO+1 (8\%) \\
\hline & & & & HOMO-4 $\rightarrow$ LUMO $(10 \%)$ \\
\hline & & & & $\mathrm{HOMO}-4 \rightarrow \mathrm{LUMO}+2(35 \%)$ \\
\hline & & & & HOMO- $1 \rightarrow$ LUMO+3 (27\%) \\
\hline \multirow{4}{*}{$\mathrm{S}_{0} \rightarrow \mathrm{S}_{24}$} & \multirow{4}{*}{2.8209} & \multirow{4}{*}{439.52} & \multirow{4}{*}{0.041} & HOMO-6 $\rightarrow$ LUMO+1 (20\%) \\
\hline & & & & HOMO-5 $\rightarrow$ LUMO $(18 \%)$ \\
\hline & & & & HOMO-5 $\rightarrow$ LUMO $+2(26 \%)$ \\
\hline & & & & HOMO-3 $\rightarrow$ LUMO+3 (16\%) \\
\hline \multirow{7}{*}{$\mathrm{S}_{0} \rightarrow \mathrm{S}_{25}$} & \multirow{7}{*}{2.8285} & \multirow{7}{*}{438.85} & \multirow{7}{*}{0.080} & HOMO-6 $\rightarrow$ LUMO (7\%) \\
\hline & & & & $\mathrm{HOMO}-6 \rightarrow \mathrm{LUMO}+2(9 \%)$ \\
\hline & & & & HOMO-5 $\rightarrow$ LUMO (6\%) \\
\hline & & & & HOMO-5 $\rightarrow$ LUMO+1 (13\%) \\
\hline & & & & $\mathrm{HOMO}-1 \rightarrow \mathrm{LUMO}+5$ (8\%) \\
\hline & & & & $\mathrm{HOMO} \rightarrow \mathrm{LUMO}+4(15 \%)$ \\
\hline & & & & $\mathrm{HOMO} \rightarrow \mathrm{LUMO}+5(15 \%)$ \\
\hline
\end{tabular}



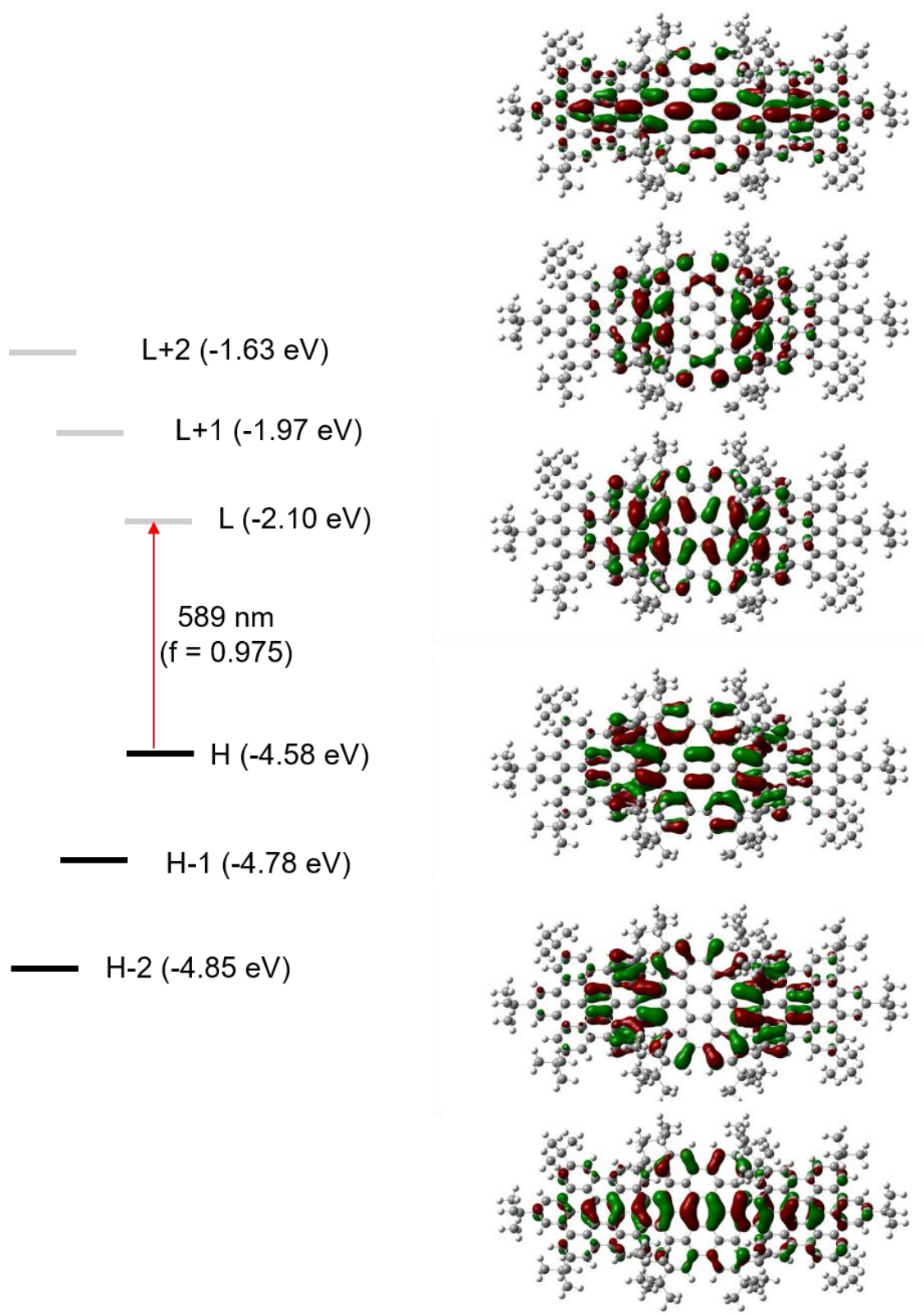

Figure S13. Kohn-Sham molecular orbitals and their energies of 2. TD-DFT result for the $\mathrm{S}_{0}-\mathrm{S}_{1}$ transition is indicated with a red arrow. 

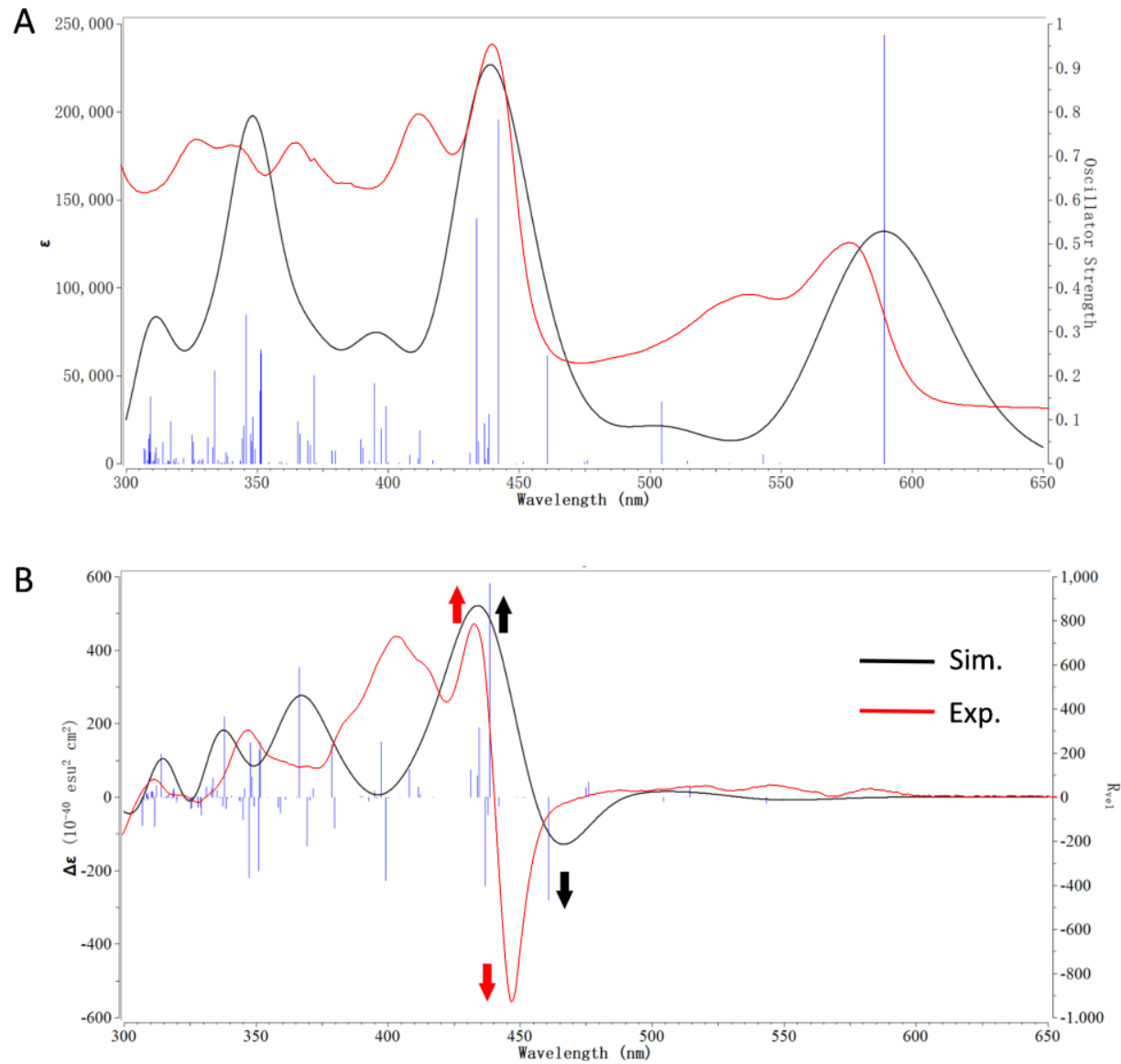

Figure S14. Computational and experimental UV-Vis absorption $(\mathrm{A})$ and $\mathrm{CD}(\mathrm{B})$ spectra of $(P)$-2. The arrows show the sign of the corresponding $\mathrm{CD}$ signals. Because of the weak experimental and computational CD signals at longer wavelength, it is difficult to compare the CD pattern in the spectral range above $500 \mathrm{~nm}$.

Table S6. TD-DFT data of 2

\begin{tabular}{llllr}
\hline Transition & $\begin{array}{l}\text { Energy } \\
(\mathrm{eV})\end{array}$ & $\begin{array}{l}\text { Wavelength } \\
(\mathrm{nm})\end{array}$ & $\begin{array}{l}\text { Oscillator } \\
\text { strength }\end{array}$ & Contribution of Orbitals \\
\hline $\mathrm{S}_{0} \rightarrow \mathrm{S}_{1}$ & 2.1037 & 589.35 & 0.975 & HOMO $\rightarrow$ LUMO $(96 \%)$ \\
\hline $\mathrm{S}_{0} \rightarrow \mathrm{S}_{2}$ & 2.2569 & 549.36 & 0.001 & HOMO $\rightarrow$ LUMO $+1(94 \%)$ \\
\hline $\mathrm{S}_{0} \rightarrow \mathrm{S}_{3}$ & 2.2824 & 543.21 & 0.021 & HOMO-2 $\rightarrow$ LUMO $(60 \%)$ \\
\hline $\mathrm{S}_{0} \rightarrow \mathrm{S}_{4}$ & 2.3382 & 530.25 & 0.000 & HOMO $\rightarrow$ LUMO $+2(25 \%)$ \\
\hline & & & HOMO-1 $\rightarrow$ LUMO $(94 \%)$ \\
& & & HOMO-3 $\rightarrow$ LUMO $(15 \%)$ \\
$\mathrm{S}_{0} \rightarrow \mathrm{S}_{5}$ & & & HOMO-2 $\rightarrow$ LUMO $+1(61 \%)$ \\
& 2.4111 & 514.22 & 0.006 & HOMO-1 $\rightarrow$ LUMO $+2(14 \%)$ \\
& & & HOMO $\rightarrow$ LUMO $+3(7 \%)$
\end{tabular}




\begin{tabular}{|c|c|c|c|c|}
\hline $\mathrm{S}_{0} \rightarrow \mathrm{S}_{6}$ & 2.4583 & 504.34 & 0.140 & HOMO- $1 \rightarrow$ LUMO+1 $(94 \%)$ \\
\hline \multirow{5}{*}{$\mathrm{S}_{0} \rightarrow \mathrm{S}_{7}$} & \multirow{5}{*}{2.6051} & \multirow{5}{*}{475.93} & \multirow{5}{*}{0.008} & HOMO-4 $\rightarrow$ LUMO (13\%) \\
\hline & & & & $\mathrm{HOMO}-4 \rightarrow \mathrm{LUMO}+1(11 \%)$ \\
\hline & & & & HOMO-3 $\rightarrow$ LUMO (44\%) \\
\hline & & & & HOMO-3 $\rightarrow$ LUMO+1 (6\%) \\
\hline & & & & HOMO-2 $\rightarrow$ LUMO+1 $(17 \%)$ \\
\hline \multirow{4}{*}{$\mathrm{S}_{0} \rightarrow \mathrm{S}_{8}$} & \multirow{4}{*}{2.6113} & \multirow{4}{*}{474.79} & \multirow{4}{*}{0.004} & HOMO-4 $\rightarrow$ LUMO $(41 \%)$ \\
\hline & & & & HOMO-3 $\rightarrow$ LUMO (13\%) \\
\hline & & & & $\mathrm{HOMO}-3 \rightarrow \mathrm{LUMO}+1(23 \%)$ \\
\hline & & & & $\mathrm{HOMO} \rightarrow \mathrm{LUMO}+6(7 \%)$ \\
\hline \multirow{3}{*}{$\mathrm{S}_{0} \rightarrow \mathrm{S}_{9}$} & \multirow{3}{*}{2.6908} & \multirow{3}{*}{460.76} & \multirow{3}{*}{0.246} & HOMO-4 $\rightarrow$ LUMO (7\%) \\
\hline & & & & HOMO-2 $\rightarrow$ LUMO $(31 \%)$ \\
\hline & & & & $\mathrm{HOMO} \rightarrow \mathrm{LUMO}+2(53 \%)$ \\
\hline \multirow{3}{*}{$\mathrm{S}_{0} \rightarrow \mathrm{S}_{10}$} & \multirow{3}{*}{2.7466} & \multirow{3}{*}{451.41} & \multirow{3}{*}{0.004} & HOMO-6 $\rightarrow$ LUMO+1 (17\%) \\
\hline & & & & HOMO-5 $\rightarrow$ LUMO $(66 \%)$ \\
\hline & & & & $\mathrm{HOMO} \rightarrow \mathrm{LUMO}+5(7 \%)$ \\
\hline \multirow{3}{*}{$\mathrm{S}_{0} \rightarrow \mathrm{S}_{11}$} & \multirow{3}{*}{2.7616} & \multirow{3}{*}{448.96} & \multirow{3}{*}{0.000} & HOMO-6 $\rightarrow$ LUMO (54\%) \\
\hline & & & & HOMO-5 $\rightarrow$ LUMO+1 (22\%) \\
\hline & & & & $\mathrm{HOMO} \rightarrow \mathrm{LUMO}+4(14 \%)$ \\
\hline \multirow{3}{*}{$\mathrm{S}_{0} \rightarrow \mathrm{S}_{12}$} & \multirow{3}{*}{2.8053} & \multirow{3}{*}{441.97} & \multirow{3}{*}{0.783} & $\mathrm{HOMO}-2 \rightarrow \mathrm{LUMO}+2(62 \%)$ \\
\hline & & & & HOMO- $1 \rightarrow$ LUMO $+2(10 \%)$ \\
\hline & & & & $\mathrm{HOMO} \rightarrow \mathrm{LUMO}+5(10 \%)$ \\
\hline \multirow{4}{*}{$\mathrm{S}_{0} \rightarrow \mathrm{S}_{13}$} & \multirow{4}{*}{2.8280} & \multirow{4}{*}{438.42} & \multirow{4}{*}{0.112} & HOMO-3 $\rightarrow$ LUMO (9\%) \\
\hline & & & & HOMO- $2 \rightarrow \mathrm{LUMO}+1(9 \%)$ \\
\hline & & & & $\mathrm{HOMO}-1 \rightarrow \mathrm{LUMO}+2(31 \%)$ \\
\hline & & & & $\mathrm{HOMO} \rightarrow \mathrm{LUMO}+3(29 \%)$ \\
\hline & & & & $\mathrm{HOMO}-4 \rightarrow \mathrm{LUMO}+1(20 \%)$ \\
\hline & & & & HOMO-3 $\rightarrow$ LUMO (7\%) \\
\hline & & & & $\mathrm{HOMO}-3 \rightarrow \mathrm{LUMO}+1(8 \%)$ \\
\hline $\mathrm{S}_{0} \rightarrow \mathrm{S}_{14}$ & 2.8315 & 437.88 & 0.036 & HOMO- $1 \rightarrow$ LUMO $+2(12 \%)$ \\
\hline & & & & HOMO-1 $\rightarrow$ LUMO+6 (5\%) \\
\hline & & & & $\mathrm{HOMO} \rightarrow \mathrm{LUMO}+3(22 \%)$ \\
\hline & & & & $\mathrm{HOMO} \rightarrow \mathrm{LUMO}+4(8 \%)$ \\
\hline & & & & HOMO-6 $\rightarrow$ LUMO (8\%) \\
\hline & & & & HOMO $-4 \rightarrow \mathrm{LUMO}+1(5 \%)$ \\
\hline $\mathrm{S}_{0} \rightarrow \mathrm{S}_{15}$ & 2.8364 & 437.11 & 0.011 & $\mathrm{HOMO}-2 \rightarrow \mathrm{LUMO}+3(5 \%)$ \\
\hline & & & & HOMO- $1 \rightarrow \mathrm{LUMO}+5$ (7\%) \\
\hline & & & & $\mathrm{HOMO} \rightarrow \mathrm{LUMO}+3(6 \%)$ \\
\hline
\end{tabular}


$\mathrm{HOMO} \rightarrow \mathrm{LUMO}+4(35 \%)$

$\mathrm{HOMO} \rightarrow \mathrm{LUMO}+5(9 \%)$

HOMO-4 $\rightarrow$ LUMO (7\%)

$\mathrm{HOMO}-3 \rightarrow \mathrm{LUMO}+1(42 \%)$

$\begin{array}{llll}\mathrm{S}_{0} \rightarrow \mathrm{S}_{16} & 2.8393 & 436.68 & 0.090\end{array}$

$\mathrm{HOMO}-1 \rightarrow \mathrm{LUMO}+2(6 \%)$

$\mathrm{HOMO} \rightarrow \mathrm{LUMO}+2(11 \%)$

$\mathrm{HOMO} \rightarrow \mathrm{LUMO}+4(8 \%)$

$\mathrm{HOMO} \rightarrow \mathrm{LUMO}+6(13 \%)$

\begin{tabular}{|c|c|c|c|c|}
\hline \multirow{3}{*}{$\mathrm{S}_{0} \rightarrow \mathrm{S}_{17}$} & \multirow{3}{*}{2.8539} & \multirow{3}{*}{434.44} & \multirow{3}{*}{0.052} & HOMO-4 $\rightarrow$ LUMO (21\%) \\
\hline & & & & HOMO- $1 \rightarrow$ LUMO+3 (12\%) \\
\hline & & & & $\mathrm{HOMO} \rightarrow \mathrm{LUMO}+6(44 \%)$ \\
\hline \multirow{4}{*}{$\mathrm{S}_{0} \rightarrow \mathrm{S}_{18}$} & \multirow{4}{*}{2.8585} & \multirow{4}{*}{433.73} & \multirow{4}{*}{0.557} & HOMO-5 $\rightarrow$ LUMO $(6 \%)$ \\
\hline & & & & HOMO- $2 \rightarrow$ LUMO+2 (21\%) \\
\hline & & & & $\mathrm{HOMO}-1 \rightarrow \mathrm{LUMO}+4(6 \%)$ \\
\hline & & & & $\mathrm{HOMO} \rightarrow \mathrm{LUMO}+5(39 \%)$ \\
\hline
\end{tabular}


DFT and TD-DFT results of 3

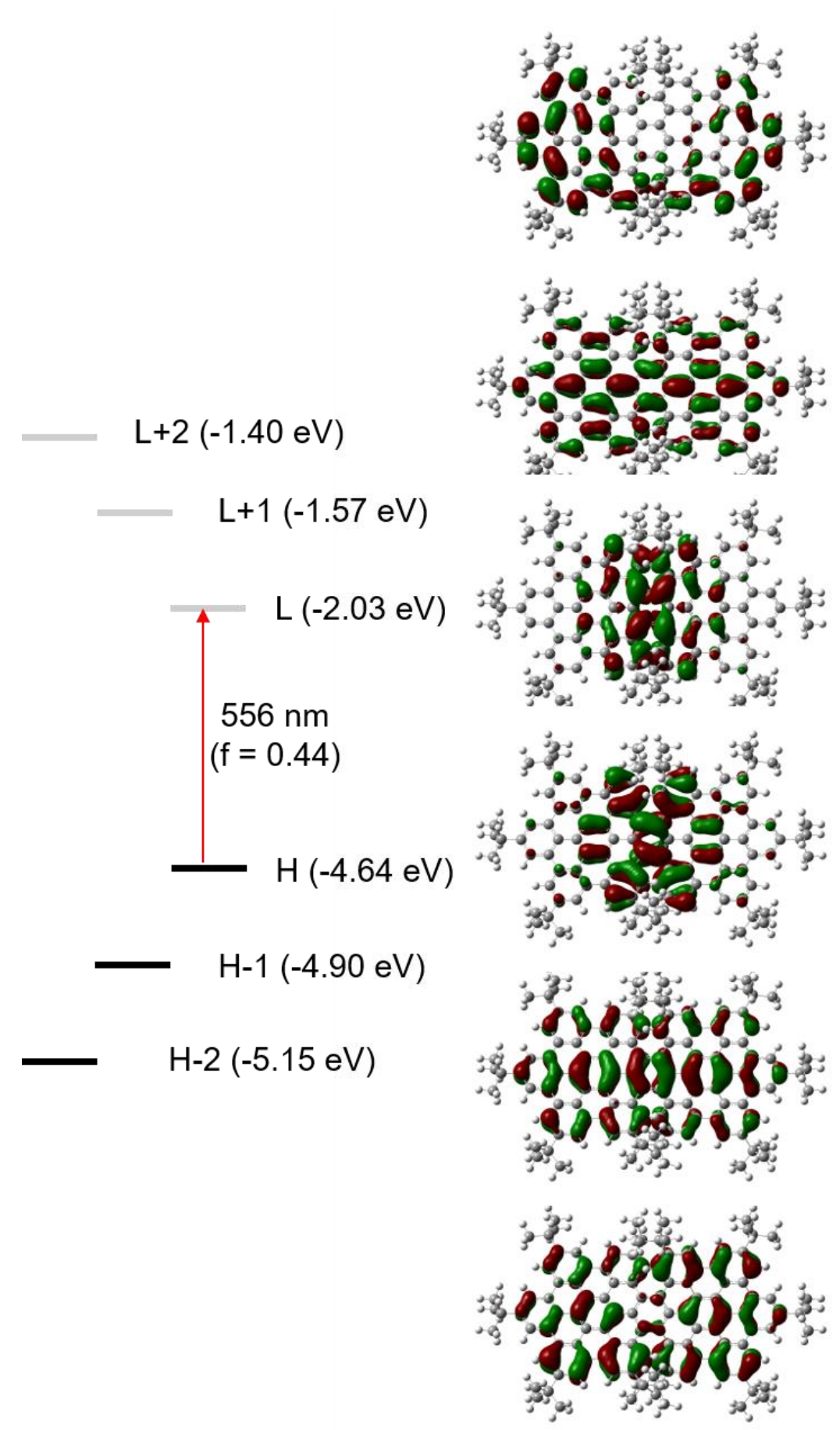

Figure S15. Kohn-Sham molecular orbitals and their energies of 3. TD-DFT result for the $\mathrm{S}_{0}-\mathrm{S}_{1}$ transition is indicated with a red arrow. 

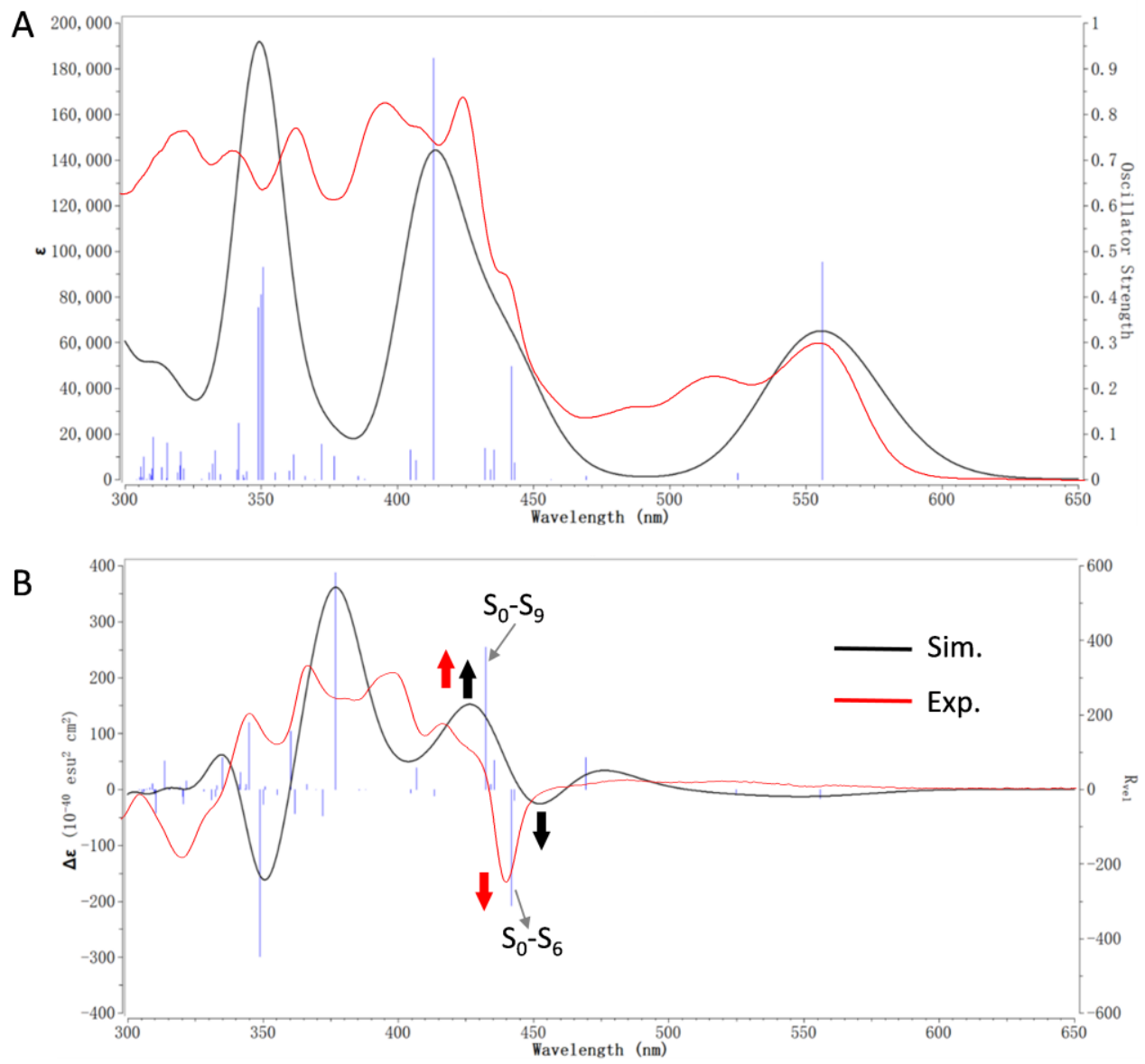

Figure S16. Computational and experimental UV-Vis absorption (A) and CD (B) spectra of $(P)-3$. The arrows show the sign of the corresponding CD signals. Because of the weak experimental and computational CD signals at longer wavelength, it is difficult to compare the CD pattern in the spectral range above $500 \mathrm{~nm}$. Note: The recorded CD signal of $\mathbf{3}$ around $441 \mathrm{~nm}$ is weaker than expected, which should be caused by the offset of two close Cotton effects of opposite signs (trasition $\mathrm{S}_{0}-\mathrm{S}_{6}$ and $\mathrm{S}_{0}-\mathrm{S}_{9}$ ) according to TD-DFT results.

Table S7. TD-DFT data of 3

\begin{tabular}{ccccc}
\hline Transition & $\begin{array}{c}\text { Energy } \\
(\mathrm{eV})\end{array}$ & $\begin{array}{c}\text { Wavelength } \\
(\mathrm{nm})\end{array}$ & $\begin{array}{c}\text { Oscillator } \\
\text { strength }\end{array}$ & Contribution of Orbitals \\
\hline $\mathrm{S}_{0} \rightarrow \mathrm{S}_{1}$ & 2.2300 & 555.98 & 0.477 & HOMO $\rightarrow$ LUMO $(97 \%)$ \\
\hline $\mathrm{S}_{0} \rightarrow \mathrm{S}_{2}$ & 2.3622 & 524.87 & 0.015 & HOMO-1 $\rightarrow$ LUMO $(70 \%)$ \\
& & & HOMO $\rightarrow$ LUMO $+1(28 \%)$ \\
\hline $\mathrm{S}_{0} \rightarrow \mathrm{S}_{3}$ & 2.6414 & 469.39 & 0.008 & HOMO-2 $\rightarrow$ LUMO $(81 \%)$ \\
& & & HOMO $\rightarrow$ LUMO $+4(10 \%)$ \\
\hline
\end{tabular}




\begin{tabular}{|c|c|c|c|c|}
\hline $\mathrm{S}_{0} \rightarrow \mathrm{S}_{4}$ & 2.7164 & 456.43 & 0.001 & HOMO-3 $\rightarrow$ LUMO $(89 \%)$ \\
\hline \multirow{3}{*}{$\mathrm{S}_{0} \rightarrow \mathrm{S}_{5}$} & \multirow{3}{*}{2.7996} & \multirow{3}{*}{442.86} & \multirow{3}{*}{0.037} & HOMO-4 $\rightarrow$ LUMO $(30 \%)$ \\
\hline & & & & $\mathrm{HOMO} \rightarrow \mathrm{LUMO}+1(8 \%)$ \\
\hline & & & & $\mathrm{HOMO} \rightarrow \mathrm{LUMO}+2(52 \%)$ \\
\hline \multirow{3}{*}{$\mathrm{S}_{0} \rightarrow \mathrm{S}_{6}$} & \multirow{3}{*}{2.8059} & \multirow{3}{*}{441.88} & \multirow{3}{*}{0.248} & HOMO-4 $\rightarrow$ LUMO (7\%) \\
\hline & & & & HOMO- $1 \rightarrow$ LUMO $(23 \%)$ \\
\hline & & & & $\mathrm{HOMO} \rightarrow \mathrm{LUMO}+1(54 \%)$ \\
\hline \multirow{4}{*}{$\mathrm{S}_{0} \rightarrow \mathrm{S}_{7}$} & \multirow{4}{*}{2.8472} & \multirow{4}{*}{435.46} & \multirow{4}{*}{0.065} & HOMO-4 $\rightarrow$ LUMO (24\%) \\
\hline & & & & HOMO-2 $\rightarrow$ LUMO+1 (17\%) \\
\hline & & & & $\mathrm{HOMO} \rightarrow \mathrm{LUMO}+2(18 \%)$ \\
\hline & & & & $\mathrm{HOMO} \rightarrow \mathrm{LUMO}+3(21 \%)$ \\
\hline \multirow{3}{*}{$\mathrm{S}_{0} \rightarrow \mathrm{S}_{8}$} & \multirow{3}{*}{2.8558} & \multirow{3}{*}{434.15} & \multirow{3}{*}{0.021} & HOMO-4 $\rightarrow$ LUMO (13\%) \\
\hline & & & & HOMO- $1 \rightarrow$ LUMO+1 (7\%) \\
\hline & & & & $\mathrm{HOMO} \rightarrow \mathrm{LUMO}+3(58 \%)$ \\
\hline \multirow{4}{*}{$\mathrm{S}_{0} \rightarrow \mathrm{S}_{9}$} & \multirow{4}{*}{2.8682} & \multirow{4}{*}{432.27} & \multirow{4}{*}{0.069} & HOMO-4 $\rightarrow$ LUMO (9\%) \\
\hline & & & & HOMO-2 $\rightarrow$ LUMO $(10 \%)$ \\
\hline & & & & HOMO- $1 \rightarrow$ LUMO+1 (11\%) \\
\hline & & & & $\mathrm{HOMO} \rightarrow \mathrm{LUMO}+4(58 \%)$ \\
\hline \multirow{3}{*}{$\mathrm{S}_{0} \rightarrow \mathrm{S}_{10}$} & \multirow{3}{*}{3.0003} & \multirow{3}{*}{413.26} & \multirow{3}{*}{0.923} & HOMO-4 $\rightarrow$ LUMO $(16 \%)$ \\
\hline & & & & HOMO- $1 \rightarrow$ LUMO+1 $(58 \%)$ \\
\hline & & & & $\mathrm{HOMO} \rightarrow \mathrm{LUMO}+2(20 \%)$ \\
\hline
\end{tabular}


Table S8. Representative experimental and theoretical CD parameters for 1-3

\begin{tabular}{cccccccccc}
\hline & $\lambda$ (exp.) & $\Delta \varepsilon$ & $\lambda$ (cal.) & $R$ & $\left|\mu_{\mathrm{e}}\right|$ & $\left|\mu_{\mathrm{m}}\right|$ & $\theta / \mathrm{deg}$ & transition & $f$ \\
\hline $\mathbf{1}$ & 450 & 760 & 471 & -643.1 & 1.64 & 1.67 & 173.68 & $\mathrm{~S}_{0}-\mathrm{S}_{16}$ & 0.175 \\
$\mathbf{2}$ & 446 & 575 & 461 & -458.0 & 1.93 & 1.01 & 174.92 & $\mathrm{~S}_{0}-\mathrm{S}_{9}$ & 0.246 \\
$\mathbf{3}$ & 441 & 160 & 442 & -308.8 & 1.90 & 0.72 & 163.84 & $\mathrm{~S}_{0}-\mathrm{S}_{6}$ & 0.248 \\
\hline
\end{tabular}

$\lambda$ (exp.): experimental absorption wavelength (nm); $\Delta \varepsilon$ : ellipticity $\left(\mathrm{M}^{-1} \mathrm{~cm}^{-1}\right) ; \lambda($ cal.): TD-DFT calculated absorption wavelength $(\mathrm{nm}) ; R$ : the rotatory strength $\left(R=\left|\mu_{\mathrm{e}}\right|\left|\mu_{\mathrm{m}}\right| \cos \theta\right) ;\left|\mu_{\mathrm{e}}\right|$ : electric transition dipole moments; $\left|\mu_{\mathrm{m}}\right|$ : magnetic transition dipole moments; $\theta$ : angels between $\boldsymbol{\mu}_{\mathrm{e}}$ and $\boldsymbol{\mu}_{\mathrm{m}} ; f$ : oscillator strength. 


\section{Cartesian coordinates of the DFT optimized structure of 1}

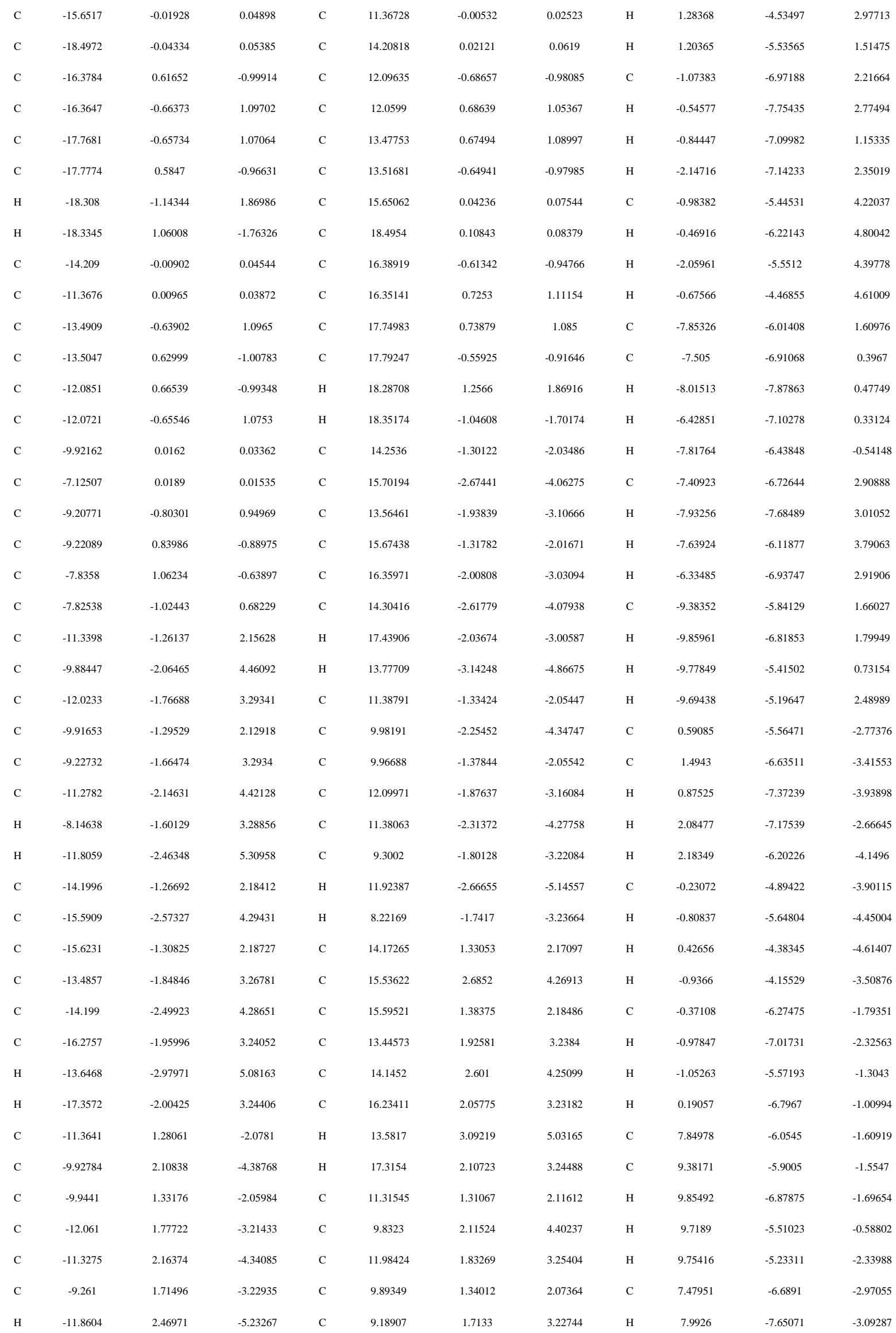




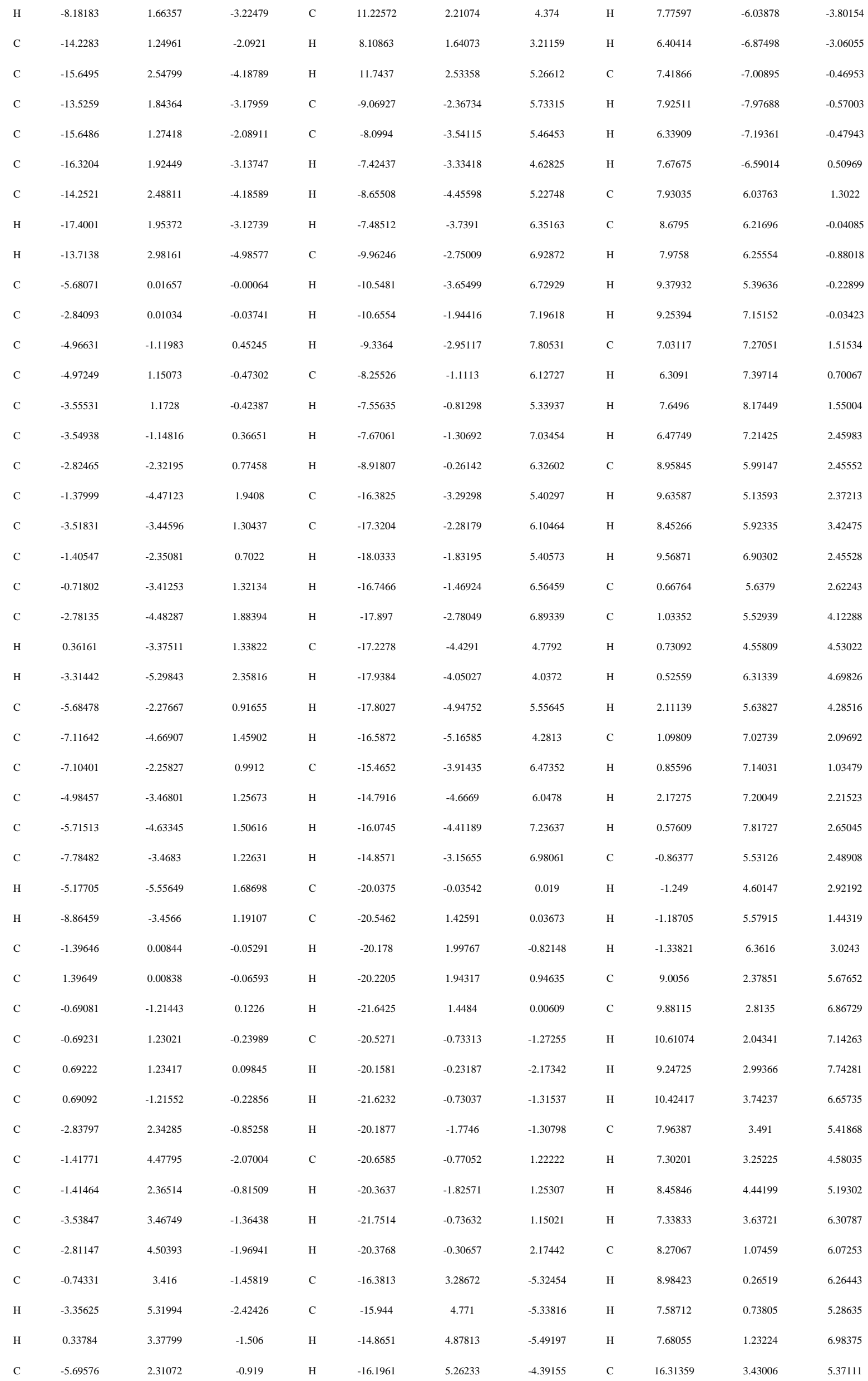




\begin{tabular}{|c|c|c|c|c|c|c|c|c|c|c|c|}
\hline C & -7.1268 & 4.7115 & -1.41711 & $\mathrm{H}$ & -16.451 & 5.31044 & -6.14764 & C & 17.25258 & 2.43821 & 6.0985 \\
\hline $\mathrm{C}$ & -5.00084 & 3.49578 & -1.28 & C & -16.0207 & 2.63363 & -6.68027 & $\mathrm{H}$ & 17.97419 & 1.98056 & 5.41372 \\
\hline $\mathrm{C}$ & -7.11923 & 2.29748 & -0.95724 & $\mathrm{H}$ & -14.9447 & 2.67286 & -6.87962 & $\mathrm{H}$ & 16.68055 & 1.63016 & 6.5685 \\
\hline C & -7.79575 & 3.5082 & -1.16938 & $\mathrm{H}$ & -16.5303 & 3.15273 & -7.50128 & $\mathrm{H}$ & 17.81942 & 2.95502 & 6.88266 \\
\hline C & -5.73301 & 4.67007 & -1.51168 & $\mathrm{H}$ & -16.3256 & 1.58117 & -6.7017 & $\mathrm{C}$ & 17.15616 & 4.56083 & 4.73399 \\
\hline $\mathrm{H}$ & -8.87674 & 3.50698 & -1.10622 & $\mathrm{C}$ & -17.9132 & 3.24237 & -5.16702 & $\mathrm{H}$ & 17.87557 & 4.17393 & 4.00472 \\
\hline $\mathrm{H}$ & -5.19226 & 5.58483 & -1.71249 & $\mathrm{H}$ & -18.2958 & 2.21533 & -5.17936 & $\mathrm{H}$ & 17.72083 & 5.09728 & 5.50642 \\
\hline C & 2.84103 & 0.00623 & -0.06559 & $\mathrm{H}$ & -18.3842 & 3.77889 & -5.99832 & $\mathrm{H}$ & 16.51458 & 5.28388 & 4.2176 \\
\hline C & 5.68085 & 0.00018 & -0.05201 & $\mathrm{H}$ & -18.2426 & 3.72112 & -4.23774 & $\mathrm{C}$ & 15.38305 & 4.0639 & 6.42272 \\
\hline C & 3.55197 & -1.16095 & -0.441 & $\mathrm{C}$ & -9.19215 & 2.42479 & -5.70411 & $\mathrm{H}$ & 14.77599 & 3.3109 & 6.93813 \\
\hline $\mathrm{C}$ & 3.55295 & 1.17223 & 0.31299 & $\mathrm{C}$ & -7.66097 & 2.43293 & -5.53174 & $\mathrm{H}$ & 14.70789 & 4.80391 & 5.97807 \\
\hline C & 4.96932 & 1.14508 & 0.38727 & $\mathrm{H}$ & -7.27739 & 1.45167 & -5.23138 & $\mathrm{H}$ & 15.98248 & 4.57923 & 7.18162 \\
\hline C & 4.96985 & -1.14079 & -0.50028 & $\mathrm{H}$ & -7.33787 & 3.16757 & -4.78567 & $\mathrm{C}$ & 20.03419 & 0.17326 & 0.12211 \\
\hline C & 2.83023 & -2.33654 & -0.84791 & $\mathrm{H}$ & -7.18495 & 2.69206 & -6.48426 & C & 20.5442 & -0.46883 & 1.43446 \\
\hline C & 1.39636 & -4.48609 & -2.0234 & $\mathrm{C}$ & -9.62053 & 3.81174 & -6.23819 & $\mathrm{H}$ & 20.24232 & -1.52036 & 1.49926 \\
\hline C & 1.40743 & -2.35804 & -0.79513 & $\mathrm{H}$ & -10.6952 & 3.86348 & -6.4415 & $\mathrm{H}$ & 20.15452 & 0.04452 & 2.31972 \\
\hline C & 3.52509 & -3.46638 & -1.3552 & $\mathrm{H}$ & -9.09822 & 4.03155 & -7.17718 & $\mathrm{H}$ & 21.63935 & -0.42542 & 1.4797 \\
\hline C & 2.79169 & -4.50912 & -1.94004 & $\mathrm{H}$ & -9.37745 & 4.60305 & -5.52098 & C & 20.48964 & 1.651 & 0.06343 \\
\hline C & 0.7288 & -3.4175 & -1.41682 & C & -9.55758 & 1.34419 & -6.75072 & $\mathrm{H}$ & 20.14868 & 2.1296 & -0.86163 \\
\hline $\mathrm{H}$ & 3.33092 & -5.33029 & -2.39206 & $\mathrm{H}$ & -10.6352 & 1.31744 & -6.94591 & $\mathrm{H}$ & 21.58427 & 1.71426 & 0.09631 \\
\hline H & -0.35284 & -3.38017 & -1.45244 & $\mathrm{H}$ & -9.25601 & 0.34841 & -6.40674 & $\mathrm{H}$ & 20.09779 & 2.23371 & 0.90364 \\
\hline C & 5.69113 & -2.3066 & -0.93466 & $\mathrm{H}$ & -9.04849 & 1.5449 & -7.70162 & C & 20.68555 & -0.57313 & -1.05782 \\
\hline C & 7.11826 & -4.70868 & -1.44354 & $\mathrm{C}$ & -7.92983 & 6.02042 & -1.54746 & $\mathrm{H}$ & 20.39097 & -0.14705 & -2.02368 \\
\hline C & 4.98965 & -3.49532 & -1.28464 & C & -7.02463 & 7.2479 & -1.76609 & $\mathrm{H}$ & 20.42871 & -1.63861 & -1.05937 \\
\hline C & 7.11259 & -2.29821 & -0.9754 & $\mathrm{H}$ & -6.44083 & 7.16729 & -2.69036 & $\mathrm{H}$ & 21.77623 & -0.49768 & -0.98381 \\
\hline C & 7.78921 & -3.5146 & -1.18628 & $\mathrm{H}$ & -6.32902 & 7.3967 & -0.93229 & $\mathrm{C}$ & 16.44782 & -3.45696 & -5.16027 \\
\hline C & 5.71904 & -4.66396 & -1.52412 & $\mathrm{H}$ & -7.64191 & 8.14979 & -1.84591 & $\mathrm{C}$ & 17.97777 & -3.40286 & -4.98757 \\
\hline $\mathrm{H}$ & 8.86752 & -3.51198 & -1.12149 & $\mathrm{C}$ & -8.74278 & 6.25128 & -0.25082 & $\mathrm{H}$ & 18.45904 & -3.9713 & -5.79134 \\
\hline $\mathrm{H}$ & 5.18115 & -5.58285 & -1.72553 & $\mathrm{H}$ & -9.4496 & 5.43741 & -0.06058 & $\mathrm{H}$ & 18.29756 & -3.84304 & -4.03617 \\
\hline C & 7.12511 & -0.0051 & -0.04214 & $\mathrm{H}$ & -9.31859 & 7.18201 & -0.32433 & $\mathrm{H}$ & 18.35867 & -2.37631 & -5.0367 \\
\hline C & 9.92168 & -0.0145 & 0.00403 & $\mathrm{H}$ & -8.07991 & 6.32891 & 0.61851 & $\mathrm{C}$ & 16.10138 & -2.86044 & -6.54543 \\
\hline C & 7.83592 & -1.06437 & -0.67058 & C & -8.89959 & 5.92 & -2.74749 & $\mathrm{H}$ & 16.62082 & -3.41179 & -7.3388 \\
\hline C & 7.82557 & 1.04854 & 0.60735 & $\mathrm{H}$ & -9.5832 & 5.07048 & -2.65377 & $\mathrm{H}$ & 16.4052 & -1.80922 & -6.60651 \\
\hline C & 9.20056 & 0.82733 & 0.89391 & $\mathrm{H}$ & -8.34415 & 5.80073 & -3.68513 & $\mathrm{H}$ & 15.02776 & -2.90959 & -6.75505 \\
\hline C & 9.22802 & -0.85386 & -0.90973 & $\mathrm{H}$ & -9.50454 & 6.83174 & -2.82651 & $\mathrm{C}$ & 16.01328 & -4.94156 & -5.11827 \\
\hline C & 5.68991 & 2.31255 & 0.8205 & C & -0.62049 & 5.54573 & -2.84432 & $\mathrm{H}$ & 16.53069 & -5.51259 & -5.89903 \\
\hline C & 7.12582 & 4.72366 & 1.25073 & $\mathrm{C}$ & -1.53099 & 6.60726 & -3.49085 & $\mathrm{H}$ & 14.93649 & -5.05731 & -5.28005 \\
\hline C & 7.11134 & 2.29449 & 0.88271 & $\mathrm{H}$ & -2.22836 & 6.16421 & -4.21098 & $\mathrm{H}$ & 16.25515 & -5.39317 & -4.14945 \\
\hline C & 4.99658 & 3.51039 & 1.1366 & $\mathrm{H}$ & -0.91776 & 7.33696 & -4.03149 & $\mathrm{C}$ & 9.26427 & -2.65471 & -5.65061 \\
\hline C & 5.73032 & 4.68985 & 1.33345 & $\mathrm{H}$ & -2.11315 & 7.15807 & -2.74299 & $\mathrm{C}$ & 9.69814 & -1.69378 & -6.78375 \\
\hline C & 7.79217 & 3.50835 & 1.06296 & $\mathrm{C}$ & 0.18818 & 4.8593 & -3.97145 & $\mathrm{H}$ & 9.20163 & -1.96341 & -7.7241 \\
\hline $\mathrm{H}$ & 5.18968 & 5.6131 & 1.49244 & $\mathrm{H}$ & -0.47717 & 4.33836 & -4.66936 & $\mathrm{H}$ & 10.77947 & -1.72743 & -6.95488 \\
\hline H & 8.8739 & 3.49934 & 1.01107 & $\mathrm{H}$ & 0.89886 & 4.12615 & -3.57692 & $\mathrm{H}$ & 9.43071 & -0.65883 & -6.54253 \\
\hline C & 2.83285 & 2.35303 & 0.70782 & $\mathrm{H}$ & 0.75916 & 5.60536 & -4.53768 & $\mathrm{C}$ & 9.64772 & -4.10255 & -6.0378 \\
\hline C & 1.40216 & 4.5225 & 1.85347 & $\mathrm{C}$ & 0.35249 & 6.26915 & -1.88504 & $\mathrm{H}$ & 9.14394 & -4.39265 & -6.96782 \\
\hline
\end{tabular}




$\begin{array}{llllllllllll}\mathrm{C} & 1.41285 & 2.38004 & 0.65242 & \mathrm{H} & -0.20042 & 6.80337 & -1.10356 & \mathrm{H} & 10.72523 & -4.21553 & -6.19719 \\ \mathrm{C} & 0.73295 & 3.4514 & 1.26249 & \mathrm{H} & 0.9549 & 7.00307 & -2.43455 & \mathrm{C} & 7.73066 & -2.58494 & -5.52091 \\ \mathrm{C} & 2.80215 & 4.53621 & 1.77509 & \mathrm{C} & -0.63651 & -5.57533 & 2.71728 & \mathrm{H} & 7.26727 & -2.89615 & -6.46401 \\ \mathrm{H} & -0.34635 & 3.4134 & 1.29468 & \mathrm{C} & 0.89315 & -5.47132 & 2.56362 & \mathrm{H} & 7.3848 & -1.56781 & -5.30565 \\ \mathrm{H} & 3.34142 & 5.36158 & 2.22487 & \mathrm{H} & 1.37403 & -6.29336 & 3.10585 & \mathrm{H} & 7.35604 & -3.24601 & -4.73145\end{array}$




\section{Cartesian coordinates of the DFT optimized structure of 2}

\begin{tabular}{|c|c|c|c|c|c|c|c|c|c|c|c|}
\hline $\mathrm{C}$ & 12.11792 & 0.97362 & 0.71919 & $\mathrm{H}$ & 5.15108 & 5.49171 & 6.25425 & C & -7.81799 & -0.99007 & 0.71057 \\
\hline $\mathrm{C}$ & 13.51574 & -0.93627 & -0.77679 & $\mathrm{C}$ & 12.10376 & 4.99703 & 3.82623 & C & -9.23722 & -0.97325 & 0.734 \\
\hline $\mathrm{C}$ & 11.39539 & -0.00586 & -0.02227 & $\mathrm{C}$ & 11.75678 & 4.86129 & 5.32811 & $\mathrm{C}$ & -9.23558 & 0.98346 & -0.75271 \\
\hline C & 13.51684 & 0.95471 & 0.67799 & $\mathrm{H}$ & 10.68116 & 4.95657 & 5.50952 & C & -7.08913 & 1.96715 & -1.50622 \\
\hline C & 14.24068 & 0.01453 & -0.06086 & $\mathrm{H}$ & 12.26271 & 5.64255 & 5.90859 & $\mathrm{C}$ & -5.6435 & 3.60012 & -3.32906 \\
\hline C & 12.11252 & -0.97435 & -0.77705 & $\mathrm{H}$ & 12.07565 & 3.88771 & 5.71746 & C & -7.78126 & 2.86534 & -2.36599 \\
\hline $\mathrm{H}$ & 14.07105 & 1.69458 & 1.24105 & $\mathrm{C}$ & 11.64814 & 6.3859 & 3.31826 & C & -5.66836 & 1.99192 & -1.47633 \\
\hline $\mathrm{H}$ & 14.0583 & -1.66683 & -1.35853 & $\mathrm{H}$ & 10.56875 & 6.52952 & 3.4335 & C & -4.98143 & 2.77926 & -2.41874 \\
\hline $\mathrm{C}$ & 9.95269 & -0.0123 & -0.0084 & $\mathrm{H}$ & 11.89001 & 6.51346 & 2.25698 & C & -7.04207 & 3.65062 & -3.2574 \\
\hline $\mathrm{C}$ & 7.11125 & -0.0201 & 0.01391 & $\mathrm{H}$ & 12.15202 & 7.18169 & 3.88056 & $\mathrm{H}$ & -3.90326 & 2.71533 & -2.44216 \\
\hline C & 9.24479 & 0.95386 & 0.75394 & $\mathrm{C}$ & 13.63549 & 4.91566 & 3.68202 & $\mathrm{H}$ & -7.56961 & 4.28732 & -3.95687 \\
\hline C & 9.23837 & -0.98278 & -0.75852 & $\mathrm{H}$ & 13.95519 & 5.0391 & 2.64096 & C & -9.95137 & 1.96897 & -1.52575 \\
\hline C & 7.81912 & -1.00415 & -0.7223 & $\mathrm{H}$ & 14.03093 & 3.96299 & 4.05273 & $\mathrm{C}$ & -11.3565 & 3.97694 & -2.97147 \\
\hline C & 7.82557 & 0.967 & 0.74014 & $\mathrm{H}$ & 14.10307 & 5.71579 & 4.26678 & C & -11.3749 & 1.99808 & -1.5141 \\
\hline C & 9.95131 & -1.95301 & -1.55314 & $\mathrm{C}$ & 15.78063 & 0.06225 & -0.05851 & C & -9.24475 & 2.92368 & -2.30683 \\
\hline C & 11.35041 & -3.9299 & -3.0465 & $\mathrm{C}$ & 16.40652 & -1.04733 & -0.92497 & C & -9.96411 & 3.91084 & -2.99714 \\
\hline C & 9.24147 & -2.89278 & -2.34914 & $\mathrm{H}$ & 16.10548 & -0.96436 & -1.97557 & C & -12.0346 & 3.00223 & -2.23338 \\
\hline $\mathrm{C}$ & 11.37489 & -1.98057 & -1.54961 & $\mathrm{H}$ & 16.13532 & -2.04721 & -0.56722 & $\mathrm{H}$ & -9.41676 & 4.66186 & -3.54881 \\
\hline C & 12.03153 & -2.96879 & -2.29352 & $\mathrm{H}$ & 17.499 & -0.97011 & -0.88883 & $\mathrm{H}$ & -13.1161 & 3.04157 & -2.21276 \\
\hline C & 9.95763 & -3.86581 & -3.06224 & $\mathrm{C}$ & 16.25442 & 1.42697 & -0.61316 & C & -11.3904 & -0.00101 & -0.01885 \\
\hline $\mathrm{H}$ & 13.11327 & -3.00574 & -2.28186 & $\mathrm{H}$ & 15.90766 & 1.57312 & -1.64247 & C & -14.2359 & -0.02654 & -0.05644 \\
\hline H & 9.40726 & -4.60744 & -3.62368 & $\mathrm{H}$ & 17.35012 & 1.47953 & -0.61212 & $\mathrm{C}$ & -12.1108 & -0.99053 & 0.71113 \\
\hline $\mathrm{C}$ & 7.08917 & -1.96158 & -1.51181 & $\mathrm{H}$ & 15.88089 & 2.26388 & -0.01395 & $\mathrm{C}$ & -12.1099 & 0.97755 & -0.75803 \\
\hline $\mathrm{C}$ & 5.6358 & -3.54973 & -3.36808 & $\mathrm{C}$ & 16.29952 & -0.11125 & 1.38895 & $\mathrm{C}$ & -13.513 & 0.93608 & -0.75861 \\
\hline $\mathrm{C}$ & 5.66897 & -1.99494 & -1.46962 & $\mathrm{H}$ & 15.92804 & 0.67538 & 2.05393 & $\mathrm{C}$ & -13.5098 & -0.97435 & 0.67026 \\
\hline C & 7.77765 & -2.83512 & -2.399 & $\mathrm{H}$ & 17.39563 & -0.0731 & 1.40812 & $\mathrm{H}$ & -14.0572 & 1.67248 & -1.33142 \\
\hline C & 7.03513 & -3.59401 & -3.31046 & $\mathrm{H}$ & 15.9852 & -1.07525 & 1.80488 & $\mathrm{H}$ & -14.0623 & -1.72362 & 1.22248 \\
\hline C & 4.97741 & -2.7651 & -2.42302 & $\mathrm{C}$ & 12.14515 & -5.00345 & -3.8143 & $\mathrm{C}$ & -9.95501 & -1.95296 & 1.51397 \\
\hline H & 7.56123 & -4.20096 & -4.03683 & $\mathrm{C}$ & 11.23042 & -5.97664 & -4.58234 & $\mathrm{C}$ & -11.3638 & -3.93514 & 2.99206 \\
\hline $\mathrm{H}$ & 3.89849 & -2.70931 & -2.43252 & $\mathrm{H}$ & 10.62068 & -5.45902 & -5.33171 & C & -9.24711 & -2.89443 & 2.31489 \\
\hline $\mathrm{C}$ & 7.10246 & 1.92015 & 1.54172 & $\mathrm{H}$ & 10.55865 & -6.52289 & -3.91037 & $\mathrm{C}$ & -11.3749 & -1.9896 & 1.49455 \\
\hline C & 5.66735 & 3.48973 & 3.4264 & $\mathrm{H}$ & 11.84169 & -6.71733 & -5.11007 & C & -12.0405 & -2.9803 & 2.23524 \\
\hline C & 7.79523 & 2.79339 & 2.42044 & $\mathrm{C}$ & 13.08122 & -4.31788 & -4.83819 & $\mathrm{C}$ & -9.9671 & -3.86648 & 3.0165 \\
\hline $\mathrm{C}$ & 5.6793 & 1.94682 & 1.51604 & $\mathrm{H}$ & 12.50606 & -3.73105 & -5.56337 & $\mathrm{H}$ & -13.1199 & -3.01169 & 2.21399 \\
\hline $\mathrm{C}$ & 5.00012 & 2.70623 & 2.48035 & $\mathrm{H}$ & 13.65852 & -5.06999 & -5.38987 & $\mathrm{H}$ & -9.42398 & -4.61134 & 3.58515 \\
\hline C & 7.061 & 3.54794 & 3.34831 & $\mathrm{H}$ & 13.79355 & -3.64236 & -4.35314 & $\mathrm{C}$ & -7.09153 & -1.95267 & 1.49803 \\
\hline $\mathrm{H}$ & 3.91913 & 2.64867 & 2.50671 & $\mathrm{C}$ & 12.99278 & -5.82928 & -2.81731 & C & -5.64597 & -3.54806 & 3.35437 \\
\hline $\mathrm{H}$ & 7.59711 & 4.156 & 4.0632 & $\mathrm{H}$ & 13.7017 & -5.20264 & -2.26616 & $\mathrm{C}$ & -5.67151 & -1.98356 & 1.46406 \\
\hline $\mathrm{C}$ & 9.96428 & 1.92651 & 1.53992 & $\mathrm{H}$ & 13.56959 & -6.59527 & -3.35012 & C & -7.78311 & -2.8323 & 2.37607 \\
\hline C & 11.37661 & 3.89714 & 3.02941 & $\mathrm{H}$ & 12.35353 & -6.33361 & -2.08377 & C & -7.04517 & -3.59399 & 3.2882 \\
\hline C & 11.38424 & 1.96518 & 1.51445 & $\mathrm{C}$ & 4.89032 & -4.30901 & -4.48274 & $\mathrm{C}$ & -4.9837 & -2.75787 & 2.41707 \\
\hline $\mathrm{C}$ & 9.25799 & 2.85903 & 2.35334 & $\mathrm{C}$ & 3.36043 & -4.25087 & -4.30853 & $\mathrm{H}$ & -7.57469 & -4.20564 & 4.00819 \\
\hline C & 9.9805 & 3.82523 & 3.0614 & $\mathrm{H}$ & 2.97929 & -3.22579 & -4.37322 & $\mathrm{H}$ & -3.9049 & -2.70038 & 2.43239 \\
\hline
\end{tabular}




\begin{tabular}{|c|c|c|c|c|c|c|c|c|c|c|c|}
\hline C & 12.05152 & 2.95072 & 2.25993 & $\mathrm{H}$ & 3.04271 & -4.67212 & -3.34817 & $\mathrm{C}$ & -4.90339 & 4.41195 & -4.40922 \\
\hline $\mathrm{H}$ & 9.43959 & 4.56294 & 3.64128 & $\mathrm{H}$ & 2.87736 & -4.82961 & -5.10398 & C & -5.32595 & 3.89835 & -5.80682 \\
\hline H & 13.13067 & 2.98562 & 2.23209 & $\mathrm{C}$ & 5.24917 & -3.66924 & -5.84603 & $\mathrm{H}$ & -4.81307 & 4.46566 & -6.59333 \\
\hline $\mathrm{C}$ & 5.66529 & -0.02174 & 0.02106 & $\mathrm{H}$ & 4.95158 & -2.61495 & -5.87406 & $\mathrm{H}$ & -6.40427 & 4.0008 & -5.96938 \\
\hline C & 2.86802 & -0.01732 & 0.02315 & $\mathrm{H}$ & 4.73267 & -4.19092 & -6.66137 & $\mathrm{H}$ & -5.0693 & 2.83996 & -5.92848 \\
\hline $\mathrm{C}$ & 4.96011 & 1.07175 & 0.59255 & $\mathrm{H}$ & 6.32535 & -3.71755 & -6.04488 & $\mathrm{C}$ & -5.27134 & 5.90942 & -4.28573 \\
\hline $\mathrm{C}$ & 4.95551 & -1.11574 & -0.5462 & $\mathrm{C}$ & 5.31381 & -5.79656 & -4.49539 & $\mathrm{H}$ & -4.75142 & 6.49245 & -5.05558 \\
\hline $\mathrm{C}$ & 3.57175 & -1.22793 & -0.2248 & $\mathrm{H}$ & 6.3868 & -5.92038 & -4.67504 & $\mathrm{H}$ & -4.98098 & 6.30646 & -3.30626 \\
\hline C & 3.57604 & 1.19056 & 0.27412 & $\mathrm{H}$ & 4.78402 & -6.33201 & -5.29253 & $\mathrm{H}$ & -6.34546 & 6.08246 & -4.41005 \\
\hline C & 1.4234 & -0.01339 & 0.01646 & $\mathrm{H}$ & 5.07581 & -6.28287 & -3.54328 & C & -3.37242 & 4.28504 & -4.29201 \\
\hline $\mathrm{C}$ & -1.41725 & -0.00636 & -0.00112 & $\mathrm{C}$ & 3.6437 & -6.18687 & 0.68088 & $\mathrm{H}$ & -2.89253 & 4.89378 & -5.0667 \\
\hline C & 0.71516 & 1.21326 & 0.04825 & $\mathrm{C}$ & 4.61688 & -6.5188 & -0.47384 & $\mathrm{H}$ & -3.03628 & 3.25167 & -4.43213 \\
\hline $\mathrm{C}$ & 0.70875 & -1.23762 & -0.0256 & $\mathrm{H}$ & 5.30401 & -5.69311 & -0.68329 & $\mathrm{H}$ & -3.00598 & 4.63334 & -3.31986 \\
\hline $\mathrm{C}$ & -0.70831 & -1.23435 & 0.03385 & $\mathrm{H}$ & 4.06423 & -6.73618 & -1.39534 & $\mathrm{C}$ & -12.1542 & 5.06683 & -3.71272 \\
\hline C & -0.70279 & 1.21688 & -0.02816 & $\mathrm{H}$ & 5.21809 & -7.40176 & -0.22401 & C & -13.0984 & 4.40335 & -4.74368 \\
\hline C & 1.44035 & 2.45581 & 0.06833 & $\mathrm{C}$ & 2.73341 & -7.40967 & 0.90439 & $\mathrm{H}$ & -13.6781 & 5.16726 & -5.2763 \\
\hline C & 2.88288 & 4.87712 & -0.28502 & $\mathrm{H}$ & 2.15111 & -7.65605 & 0.00896 & $\mathrm{H}$ & -13.8086 & 3.71947 & -4.26736 \\
\hline $\mathrm{C}$ & 2.86019 & 2.45743 & 0.13221 & $\mathrm{H}$ & 2.03614 & -7.25404 & 1.73554 & $\mathrm{H}$ & -12.5292 & 3.83014 & -5.48431 \\
\hline $\mathrm{C}$ & 0.74624 & 3.6943 & -0.02977 & $\mathrm{H}$ & 3.3468 & -8.28453 & 1.14768 & $\mathrm{C}$ & -12.9936 & 5.87431 & -2.69399 \\
\hline C & 1.48215 & 4.86822 & -0.216 & $\mathrm{C}$ & 4.45242 & -5.94926 & 1.97896 & $\mathrm{H}$ & -13.5726 & 6.65158 & -3.20777 \\
\hline C & 3.54621 & 3.66818 & -0.08273 & $\mathrm{H}$ & 5.16228 & -5.12279 & 1.87301 & $\mathrm{H}$ & -12.3486 & 6.363 & -1.95497 \\
\hline $\mathrm{H}$ & 0.94864 & 5.79885 & -0.36899 & $\mathrm{H}$ & 5.02422 & -6.8482 & 2.24012 & $\mathrm{H}$ & -13.7 & 5.23815 & -2.15053 \\
\hline $\mathrm{H}$ & 4.6255 & 3.63841 & -0.12134 & $\mathrm{H}$ & 3.7871 & -5.71351 & 2.81735 & $\mathrm{C}$ & -11.2427 & 6.05336 & -4.46749 \\
\hline $\mathrm{C}$ & -1.42123 & 2.46319 & -0.04746 & $\mathrm{C}$ & -3.66427 & -6.16596 & -0.70696 & $\mathrm{H}$ & -11.8561 & 6.80529 & -4.97659 \\
\hline $\mathrm{C}$ & -2.84826 & 4.89627 & 0.28529 & $\mathrm{C}$ & -4.64169 & -6.49964 & 0.44366 & $\mathrm{H}$ & -10.6388 & 5.54953 & -5.23086 \\
\hline $\mathrm{C}$ & -0.71946 & 3.69875 & 0.03703 & $\mathrm{H}$ & -5.32548 & -5.67195 & 0.65608 & $\mathrm{H}$ & -10.5657 & 6.58522 & -3.78931 \\
\hline C & -2.84139 & 2.4719 & -0.10318 & $\mathrm{H}$ & -4.09218 & -6.72468 & 1.36521 & $\mathrm{C}$ & -15.7756 & -0.0789 & -0.05627 \\
\hline $\mathrm{C}$ & -3.51893 & 3.68781 & 0.10947 & $\mathrm{H}$ & -5.24642 & -7.3784 & 0.18758 & $\mathrm{C}$ & -16.2449 & -1.43776 & -0.62884 \\
\hline $\mathrm{C}$ & -1.44792 & 4.87935 & 0.20781 & $\mathrm{C}$ & -4.46885 & -5.91723 & -2.00552 & $\mathrm{H}$ & -15.8965 & -1.56985 & -1.65948 \\
\hline H & -4.59781 & 3.66431 & 0.15864 & $\mathrm{H}$ & -5.17489 & -5.08788 & -1.89659 & $\mathrm{H}$ & -17.3404 & -1.49338 & -0.62986 \\
\hline H & -0.90907 & 5.81011 & 0.34072 & $\mathrm{H}$ & -5.04445 & -6.81189 & -2.27295 & $\mathrm{H}$ & -15.8698 & -2.2812 & -0.03985 \\
\hline $\mathrm{C}$ & -2.86202 & -0.00308 & -0.00856 & $\mathrm{H}$ & -3.80046 & -5.68011 & -2.84109 & $\mathrm{C}$ & -16.404 & 1.03987 & -0.90903 \\
\hline $\mathrm{C}$ & -5.66021 & 0.0024 & -0.00927 & $\mathrm{C}$ & -2.75935 & -7.39187 & -0.93518 & $\mathrm{H}$ & -16.1015 & 0.97141 & -1.96028 \\
\hline C & -3.56508 & 1.20987 & -0.24876 & $\mathrm{H}$ & -2.05965 & -7.23518 & -1.76409 & $\mathrm{H}$ & -16.1362 & 2.03586 & -0.53808 \\
\hline $\mathrm{C}$ & -3.57087 & -1.21296 & 0.2297 & $\mathrm{H}$ & -3.37641 & -8.26248 & -1.18438 & $\mathrm{H}$ & -17.4963 & 0.9589 & -0.87511 \\
\hline $\mathrm{C}$ & -4.95414 & -1.09842 & 0.54962 & $\mathrm{H}$ & -2.18005 & -7.64579 & -0.03989 & $\mathrm{C}$ & -16.2964 & 0.07447 & 1.3928 \\
\hline $\mathrm{C}$ & -4.95098 & 1.10014 & -0.56871 & $\mathrm{C}$ & -3.58065 & 6.21768 & 0.58679 & $\mathrm{H}$ & -15.9855 & 1.03404 & 1.82133 \\
\hline C & -1.43103 & -2.47761 & 0.0389 & $\mathrm{C}$ & -5.11278 & 6.05775 & 0.56381 & $\mathrm{H}$ & -15.9231 & -0.71945 & 2.04802 \\
\hline C & -2.86164 & -4.89777 & -0.35695 & $\mathrm{H}$ & -5.58666 & 7.02581 & 0.76207 & $\mathrm{H}$ & -17.3924 & 0.03268 & 1.41041 \\
\hline $\mathrm{C}$ & -2.85421 & -2.47962 & 0.08049 & $\mathrm{H}$ & -5.46216 & 5.35846 & 1.33153 & $\mathrm{C}$ & -12.0888 & -5.04074 & 3.7828 \\
\hline $\mathrm{C}$ & -0.73618 & -3.71332 & -0.04817 & $\mathrm{H}$ & -5.47255 & 5.70325 & -0.40857 & $\mathrm{C}$ & -13.6211 & -4.95414 & 3.64885 \\
\hline $\mathrm{C}$ & -1.46797 & -4.89288 & -0.25272 & $\mathrm{C}$ & -3.19403 & 7.28462 & -0.46455 & $\mathrm{H}$ & -13.9475 & -5.06685 & 2.60865 \\
\hline $\mathrm{C}$ & -3.53066 & -3.68571 & -0.15665 & $\mathrm{H}$ & -3.70866 & 8.22969 & -0.25275 & $\mathrm{H}$ & -14.0118 & -4.00406 & 4.03105 \\
\hline $\mathrm{H}$ & -0.92678 & -5.81863 & -0.39297 & $\mathrm{H}$ & -3.4768 & 6.9636 & -1.47366 & $\mathrm{H}$ & -14.0872 & -5.75865 & 4.22882 \\
\hline $\mathrm{H}$ & -4.61161 & -3.66183 & -0.21586 & $\mathrm{H}$ & -2.1182 & 7.48883 & -0.46927 & $\mathrm{C}$ & -11.6397 & -6.42579 & 3.25879 \\
\hline $\mathrm{C}$ & 1.42619 & -2.48389 & -0.03908 & $\mathrm{C}$ & -3.16671 & 6.71628 & 1.99241 & $\mathrm{H}$ & -11.8888 & -6.54276 & 2.197 \\
\hline
\end{tabular}




\begin{tabular}{|c|c|c|c|c|c|c|c|c|c|c|c|}
\hline C & 2.8464 & -4.91292 & 0.33975 & $\mathrm{H}$ & -3.67369 & 7.66034 & 2.22747 & $\mathrm{H}$ & -12.1418 & -7.22568 & 3.81684 \\
\hline $\mathrm{C}$ & 0.72592 & -3.71697 & 0.04097 & $\mathrm{H}$ & -2.08742 & 6.88983 & 2.0613 & $\mathrm{H}$ & -10.5599 & -6.57307 & 3.36552 \\
\hline C & 2.84943 & -2.49216 & -0.08297 & $\mathrm{H}$ & -3.43692 & 5.98442 & 2.76198 & C & -11.7323 & -4.92016 & 5.28375 \\
\hline C & 3.52072 & -3.70283 & 0.14534 & C & 3.62402 & 6.18417 & -0.6269 & $\mathrm{H}$ & -12.0461 & -3.94947 & 5.68427 \\
\hline C & 1.45258 & -4.90103 & 0.23769 & C & 3.26241 & 6.5973 & -2.07438 & $\mathrm{H}$ & -10.6558 & -5.02002 & 5.45767 \\
\hline H & 4.60191 & -3.68423 & 0.20227 & $\mathrm{H}$ & 3.77494 & 7.52863 & -2.34575 & $\mathrm{H}$ & -12.2367 & -5.70554 & 5.85993 \\
\hline H & 0.90736 & -5.82515 & 0.37306 & $\mathrm{H}$ & 2.18552 & 6.76011 & -2.1918 & C & -4.90579 & -4.31171 & 4.4695 \\
\hline C & 4.86396 & 4.22336 & 4.51777 & $\mathrm{H}$ & 3.56284 & 5.82279 & -2.78905 & C & -5.27243 & -3.67834 & 5.83371 \\
\hline C & 3.89585 & 5.23486 & 3.86189 & C & 5.15447 & 6.03093 & -0.53551 & $\mathrm{H}$ & -4.97571 & -2.62401 & 5.86809 \\
\hline H & 3.21268 & 4.75186 & 3.15635 & $\mathrm{H}$ & 5.63416 & 6.99131 & -0.75598 & $\mathrm{H}$ & -4.76001 & -4.20336 & 6.64948 \\
\hline $\mathrm{H}$ & 4.45247 & 6.00486 & 3.31492 & $\mathrm{H}$ & 5.53698 & 5.30145 & -1.25786 & $\mathrm{H}$ & -6.34965 & -3.72815 & 6.02656 \\
\hline H & 3.29036 & 5.73509 & 4.6278 & $\mathrm{H}$ & 5.47454 & 5.71908 & 0.46498 & C & -5.32787 & -5.7997 & 4.4733 \\
\hline C & 4.04913 & 3.18986 & 5.33227 & C & 3.19755 & 7.3117 & 0.3422 & $\mathrm{H}$ & -5.0847 & -6.28151 & 3.52022 \\
\hline H & 3.34276 & 2.63869 & 4.70343 & $\mathrm{H}$ & 3.72354 & 8.24132 & 0.09326 & $\mathrm{H}$ & -6.40164 & -5.9254 & 4.64709 \\
\hline $\mathrm{H}$ & 3.47259 & 3.69441 & 6.11729 & $\mathrm{H}$ & 3.4377 & 7.05274 & 1.37897 & $\mathrm{H}$ & -4.80161 & -6.33823 & 5.27068 \\
\hline H & 4.71076 & 2.45953 & 5.81182 & $\mathrm{H}$ & 2.12368 & 7.51896 & 0.28945 & C & -3.37508 & -4.2515 & 4.30344 \\
\hline C & 5.76872 & 4.99468 & 5.49777 & C & -7.10577 & 0.00316 & -0.00942 & $\mathrm{H}$ & -2.9952 & -3.22639 & 4.37458 \\
\hline H & 6.35387 & 5.77076 & 4.99081 & $\mathrm{C}$ & -9.94749 & 0.00386 & -0.01145 & $\mathrm{H}$ & -3.05216 & -4.66828 & 3.34285 \\
\hline $\mathrm{H}$ & 6.46282 & 4.32931 & 6.02388 & C & -7.81628 & 0.99938 & -0.7274 & $\mathrm{H}$ & -2.89558 & -4.83331 & 5.09881 \\
\hline
\end{tabular}




\section{Cartesian coordinates of the DFT optimized structure of 3}

\begin{tabular}{|c|c|c|c|c|c|c|c|c|c|c|c|}
\hline C & 4.96932 & -1.18709 & -0.38347 & C & -7.85768 & 1.1419 & -0.37599 & $\mathrm{H}$ & 2.10616 & -7.16964 & -2.34463 \\
\hline $\mathrm{C}$ & 3.55715 & 1.16307 & 0.34456 & $\mathrm{C}$ & -7.85233 & -1.20235 & 0.35615 & $\mathrm{H}$ & 0.50485 & -7.76561 & -2.79348 \\
\hline C & 5.68412 & -0.01881 & -0.00856 & C & -9.25544 & -1.17945 & 0.32686 & $\mathrm{H}$ & 0.78326 & -7.10268 & -1.17153 \\
\hline $\mathrm{C}$ & 3.55042 & -1.19145 & -0.35068 & $\mathrm{C}$ & -9.25672 & 1.09692 & -0.38683 & C & -0.90896 & -5.46249 & -2.61927 \\
\hline C & 2.84263 & -0.01294 & 0.0008 & $\mathrm{H}$ & -9.79837 & -2.07175 & 0.60165 & $\mathrm{H}$ & -1.28071 & -4.52271 & -3.04222 \\
\hline C & 4.97683 & 1.15302 & 0.36758 & $\mathrm{H}$ & -9.81029 & 1.97931 & -0.68088 & $\mathrm{H}$ & -1.23601 & -5.51865 & -1.57496 \\
\hline $\mathrm{C}$ & 7.1271 & -0.02384 & -0.00828 & $\mathrm{C}$ & -5.70335 & 2.33453 & -0.76956 & $\mathrm{H}$ & -1.39235 & -6.28047 & -3.16544 \\
\hline C & 9.97295 & -0.0392 & 0.00961 & C & -7.11727 & 4.70269 & -1.47056 & C & 0.99269 & -5.47214 & -4.2476 \\
\hline C & 7.84356 & -1.19713 & -0.3717 & $\mathrm{C}$ & -4.99732 & 3.49833 & -1.18904 & $\mathrm{H}$ & 2.06939 & -5.59357 & -4.40865 \\
\hline $\mathrm{C}$ & 7.85082 & 1.14726 & 0.35975 & C & -7.12369 & 2.35563 & -0.75119 & $\mathrm{H}$ & 0.70358 & -4.49405 & -4.64838 \\
\hline C & 9.24991 & 1.10435 & 0.36007 & C & -7.79184 & 3.54118 & -1.09926 & $\mathrm{H}$ & 0.47632 & -6.24525 & -4.83014 \\
\hline C & 9.24687 & -1.17226 & -0.35264 & C & -5.72024 & 4.65085 & -1.51255 & C & -0.57911 & -5.54145 & 2.78315 \\
\hline $\mathrm{H}$ & 9.8044 & 1.98779 & 0.64915 & $\mathrm{H}$ & -8.87125 & 3.56103 & -1.06722 & C & 0.38947 & -6.25726 & 1.8135 \\
\hline $\mathrm{H}$ & 9.78908 & -2.06387 & -0.63107 & $\mathrm{H}$ & -5.17986 & 5.54897 & -1.78523 & $\mathrm{H}$ & 1.06869 & -5.55631 & 1.31837 \\
\hline C & 5.69781 & 2.33806 & 0.76658 & C & -5.69204 & -2.37415 & 0.7949 & $\mathrm{H}$ & -0.16708 & -6.79079 & 1.03408 \\
\hline C & 7.11393 & 4.70643 & 1.46238 & C & -7.09229 & -4.74093 & 1.5257 & $\mathrm{H}$ & 0.99932 & -6.99061 & 2.35552 \\
\hline C & 7.11801 & 2.3601 & 0.74057 & C & -7.11536 & -2.40506 & 0.76142 & C & 0.23559 & -4.85273 & 3.90449 \\
\hline C & 4.99316 & 3.50151 & 1.18959 & C & -4.98316 & -3.52474 & 1.23728 & $\mathrm{H}$ & 0.93818 & -4.11431 & 3.50533 \\
\hline C & 5.7172 & 4.65407 & 1.51068 & C & -5.70058 & -4.68234 & 1.57681 & $\mathrm{H}$ & 0.81631 & -5.59657 & 4.46369 \\
\hline $\mathrm{C}$ & 7.78724 & 3.54557 & 1.08657 & C & -7.77245 & -3.58614 & 1.12649 & $\mathrm{H}$ & -0.42653 & -4.33752 & 4.60969 \\
\hline $\mathrm{H}$ & 5.17781 & 5.55176 & 1.78668 & $\mathrm{H}$ & -5.15172 & -5.56709 & 1.86651 & C & -1.47791 & -6.60936 & 3.43543 \\
\hline $\mathrm{H}$ & 8.86648 & 3.56591 & 1.0498 & $\mathrm{H}$ & -8.85386 & -3.61965 & 1.09428 & $\mathrm{H}$ & -2.06329 & -7.16215 & 2.69152 \\
\hline $\mathrm{C}$ & 5.68204 & -2.3724 & -0.79408 & C & 0.65972 & 5.62216 & 2.67555 & $\mathrm{H}$ & -2.17154 & -6.17164 & 4.16244 \\
\hline C & 7.08093 & -4.73813 & -1.53142 & C & 1.02985 & 6.98788 & 2.05029 & $\mathrm{H}$ & -0.85596 & -7.33653 & 3.96948 \\
\hline $\mathrm{C}$ & 4.97232 & -3.52472 & -1.22941 & $\mathrm{H}$ & 0.73852 & 7.02901 & 0.99444 & C & -7.88825 & -6.00953 & 1.88721 \\
\hline $\mathrm{C}$ & 7.10543 & -2.40114 & -0.77062 & $\mathrm{H}$ & 2.1045 & 7.19053 & 2.1073 & C & -6.9757 & -7.17998 & 2.30048 \\
\hline $\mathrm{C}$ & 7.76191 & -3.58187 & -1.13903 & $\mathrm{H}$ & 0.51226 & 7.79894 & 2.57677 & $\mathrm{H}$ & -6.29575 & -7.47315 & 1.49244 \\
\hline $\mathrm{C}$ & 5.68849 & -4.68159 & -1.57185 & $\mathrm{C}$ & 1.08527 & 5.61219 & 4.1636 & $\mathrm{H}$ & -7.5881 & -8.05414 & 2.54871 \\
\hline $\mathrm{H}$ & 8.84356 & -3.61358 & -1.11433 & $\mathrm{H}$ & 2.16431 & 5.76033 & 4.27967 & $\mathrm{H}$ & -6.37466 & -6.93725 & 3.18423 \\
\hline $\mathrm{H}$ & 5.13848 & -5.56774 & -1.85514 & $\mathrm{H}$ & 0.8266 & 4.65877 & 4.63784 & C & -8.83756 & -5.70231 & 3.07005 \\
\hline $\mathrm{C}$ & 1.39721 & -0.0106 & 0.00564 & $\mathrm{H}$ & 0.57608 & 6.41444 & 4.71189 & $\mathrm{H}$ & -9.54827 & -4.90512 & 2.82828 \\
\hline C & -1.40434 & -0.01001 & 0.01701 & C & -0.87175 & 5.46711 & 2.61105 & $\mathrm{H}$ & -8.27219 & -5.38784 & 3.95466 \\
\hline C & 0.69055 & 1.21084 & 0.17448 & $\mathrm{H}$ & -1.23981 & 5.4672 & 1.57897 & $\mathrm{H}$ & -9.41679 & -6.5952 & 3.3357 \\
\hline $\mathrm{C}$ & 0.68736 & -1.23197 & -0.15618 & $\mathrm{H}$ & -1.34851 & 6.30328 & 3.13501 & C & -8.72217 & -6.46137 & 0.66455 \\
\hline C & -0.69507 & -1.23015 & 0.18548 & $\mathrm{H}$ & -1.20951 & 4.5429 & 3.09294 & $\mathrm{H}$ & -8.07347 & -6.69375 & -0.18767 \\
\hline C & -0.6971 & 1.21073 & -0.15534 & C & 7.84245 & 6.01697 & 1.81575 & $\mathrm{H}$ & -9.42936 & -5.68931 & 0.34388 \\
\hline C & 2.83329 & 2.33695 & 0.75963 & C & 7.39316 & 7.1333 & 0.84283 & $\mathrm{H}$ & -9.29973 & -7.36154 & 0.9084 \\
\hline C & 1.39535 & 4.49059 & 1.93278 & $\mathrm{H}$ & 6.31396 & 7.31123 & 0.89472 & C & -0.66006 & 5.61687 & -2.66554 \\
\hline $\mathrm{C}$ & 3.52878 & 3.46911 & 1.26827 & $\mathrm{H}$ & 7.6384 & 6.87219 & -0.1929 & C & 0.87124 & 5.46199 & -2.59688 \\
\hline C & 1.41253 & 2.35325 & 0.72834 & $\mathrm{H}$ & 7.89828 & 8.07642 & 1.08518 & $\mathrm{H}$ & 1.3493 & 6.29715 & -3.12128 \\
\hline C & 0.72957 & 3.41333 & 1.35319 & C & 7.49066 & 6.42815 & 3.26547 & $\mathrm{H}$ & 1.21022 & 4.53685 & -3.07612 \\
\hline C & 2.79412 & 4.50938 & 1.84642 & $\mathrm{H}$ & 6.41481 & 6.58417 & 3.39662 & $\mathrm{H}$ & 1.23673 & 5.46409 & -1.56389 \\
\hline H & -0.34861 & 3.36366 & 1.3991 & $\mathrm{H}$ & 7.99732 & 7.36441 & 3.53002 & C & -1.03186 & 6.98374 & -2.04378 \\
\hline
\end{tabular}




\begin{tabular}{|c|c|c|c|c|c|c|c|c|c|c|c|}
\hline $\mathrm{H}$ & 3.32491 & 5.34249 & 2.29044 & $\mathrm{H}$ & 7.80511 & 5.65768 & 3.97863 & $\mathrm{H}$ & -0.51301 & 7.79387 & -2.57047 \\
\hline C & 2.82051 & -2.36019 & -0.76794 & C & 9.3743 & 5.88595 & 1.71633 & $\mathrm{H}$ & -0.74323 & 7.02687 & -0.98728 \\
\hline $\mathrm{C}$ & 1.36679 & -4.48839 & -1.96723 & $\mathrm{H}$ & 9.69751 & 5.62814 & 0.70129 & $\mathrm{H}$ & -2.10639 & 7.18616 & -2.10389 \\
\hline C & 1.40036 & -2.37478 & -0.72147 & $\mathrm{H}$ & 9.7652 & 5.12798 & 2.40483 & C & -1.0818 & 5.6041 & -4.15466 \\
\hline C & 3.50881 & -3.48606 & -1.2998 & $\mathrm{H}$ & 9.84302 & 6.84121 & 1.97812 & $\mathrm{H}$ & -0.57118 & 6.4053 & -4.70315 \\
\hline $\mathrm{C}$ & 2.76591 & -4.51164 & -1.89537 & $\mathrm{C}$ & 11.51309 & -0.00794 & 0.03612 & $\mathrm{H}$ & -2.16054 & 5.75203 & -4.27379 \\
\hline C & 0.70849 & -3.4248 & -1.35279 & C & 11.99712 & 0.31837 & 1.46922 & $\mathrm{H}$ & -0.82193 & 4.64978 & -4.62642 \\
\hline H & 3.29135 & -5.33331 & -2.36604 & $\mathrm{H}$ & 11.62541 & 1.2883 & 1.81572 & $\mathrm{C}$ & -7.84458 & 6.01337 & -1.82587 \\
\hline $\mathrm{H}$ & -0.37033 & -3.37407 & -1.3832 & $\mathrm{H}$ & 13.09308 & 0.35031 & 1.5035 & $\mathrm{C}$ & -7.48642 & 6.42606 & -3.2736 \\
\hline C & -3.55965 & -1.1913 & 0.36568 & $\mathrm{H}$ & 11.6564 & -0.44255 & 2.18056 & $\mathrm{H}$ & -7.99213 & 7.36244 & -3.53952 \\
\hline $\mathrm{C}$ & -4.98363 & 1.15031 & -0.36583 & C & 12.13752 & -1.35263 & -0.38285 & $\mathrm{H}$ & -7.79747 & 5.6562 & -3.98891 \\
\hline C & -2.85041 & -0.01293 & 0.01582 & $\mathrm{H}$ & 11.85949 & -1.63141 & -1.40573 & $\mathrm{H}$ & -6.41003 & 6.58247 & -3.39977 \\
\hline C & -4.97853 & -1.18866 & 0.38826 & $\mathrm{H}$ & 11.84188 & -2.1672 & 0.28823 & C & -9.37681 & 5.88181 & -1.73342 \\
\hline C & -5.69216 & -0.02126 & 0.00702 & $\mathrm{H}$ & 13.23019 & -1.27773 & -0.34781 & $\mathrm{H}$ & -9.84465 & 6.8372 & -1.99632 \\
\hline C & -3.56359 & 1.16138 & -0.33482 & $\mathrm{C}$ & 12.02361 & 1.08065 & -0.93797 & $\mathrm{H}$ & -9.70446 & 5.62285 & -0.7201 \\
\hline $\mathrm{C}$ & -2.83852 & 2.33503 & -0.7491 & $\mathrm{H}$ & 11.65358 & 2.07571 & -0.66979 & $\mathrm{H}$ & -9.7644 & 5.12443 & -2.42443 \\
\hline C & -1.39759 & 4.48675 & -1.92248 & $\mathrm{H}$ & 11.70118 & 0.87054 & -1.96412 & C & -7.39995 & 7.12889 & -0.84989 \\
\hline $\mathrm{C}$ & -1.41785 & 2.35187 & -0.71346 & $\mathrm{H}$ & 13.11983 & 1.11892 & -0.92644 & $\mathrm{H}$ & -7.90425 & 8.0721 & -1.09359 \\
\hline $\mathrm{C}$ & -3.53261 & 3.46606 & -1.26226 & C & 7.87588 & -6.00632 & -1.89654 & $\mathrm{H}$ & -6.32057 & 7.30717 & -0.89681 \\
\hline C & -2.79661 & 4.50538 & -1.84024 & C & 8.81782 & -5.69947 & -3.08535 & $\mathrm{H}$ & -7.64972 & 6.86672 & 0.18448 \\
\hline $\mathrm{C}$ & -0.73339 & 3.41087 & -1.33873 & $\mathrm{H}$ & 9.52939 & -4.90156 & -2.84859 & C & -11.5205 & -0.01885 & -0.07963 \\
\hline $\mathrm{H}$ & -3.32643 & 5.33722 & -2.28779 & $\mathrm{H}$ & 8.24693 & -5.38621 & -3.96685 & $\mathrm{C}$ & -12.146 & -1.36412 & 0.33599 \\
\hline $\mathrm{H}$ & 0.34487 & 3.36129 & -1.38188 & $\mathrm{H}$ & 9.3961 & -6.59218 & -3.35365 & $\mathrm{H}$ & -11.8441 & -2.17889 & -0.33206 \\
\hline C & -2.83253 & -2.35858 & 0.79151 & $\mathrm{C}$ & 8.7175 & -6.45615 & -0.67843 & $\mathrm{H}$ & -13.2384 & -1.29095 & 0.29274 \\
\hline C & -1.38855 & -4.47714 & 2.01718 & $\mathrm{H}$ & 9.42572 & -5.68298 & -0.36277 & $\mathrm{H}$ & -11.8751 & -1.64146 & 1.36118 \\
\hline C & -3.5213 & -3.4828 & 1.31831 & $\mathrm{H}$ & 9.29455 & -7.35596 & -0.92481 & $\mathrm{C}$ & -11.9941 & 0.30519 & -1.51673 \\
\hline C & -1.40937 & -2.37016 & 0.75641 & $\mathrm{H}$ & 8.07412 & -6.68837 & 0.17786 & $\mathrm{H}$ & -11.6467 & -0.45589 & -2.22464 \\
\hline $\mathrm{C}$ & -0.72586 & -3.41227 & 1.39939 & $\mathrm{C}$ & 6.96187 & -7.17795 & -2.30322 & $\mathrm{H}$ & -11.6213 & 1.27536 & -1.86136 \\
\hline C & -2.7819 & -4.50941 & 1.92712 & $\mathrm{H}$ & 6.35533 & -6.93636 & -3.18357 & $\mathrm{H}$ & -13.0898 & 0.33531 & -1.55944 \\
\hline $\mathrm{H}$ & 0.35502 & -3.36394 & 1.44243 & $\mathrm{H}$ & 6.28717 & -7.4712 & -1.49083 & $\mathrm{C}$ & -12.0402 & 1.06989 & 0.8894 \\
\hline $\mathrm{H}$ & -3.31396 & -5.32898 & 2.38891 & $\mathrm{H}$ & 7.57351 & -8.05174 & -2.55458 & $\mathrm{H}$ & -11.6698 & 2.0653 & 0.62297 \\
\hline C & -7.135 & -0.02816 & -0.00275 & $\mathrm{C}$ & 0.62134 & -5.58722 & -2.74916 & $\mathrm{H}$ & -11.7252 & 0.8614 & 1.91818 \\
\hline $\mathrm{C}$ & -9.98055 & -0.04759 & -0.04138 & $\mathrm{C}$ & 1.03272 & -6.98552 & -2.23162 & $\mathrm{H}$ & -13.1364 & 1.10631 & 0.86951 \\
\hline
\end{tabular}




\section{Electrochemical results}
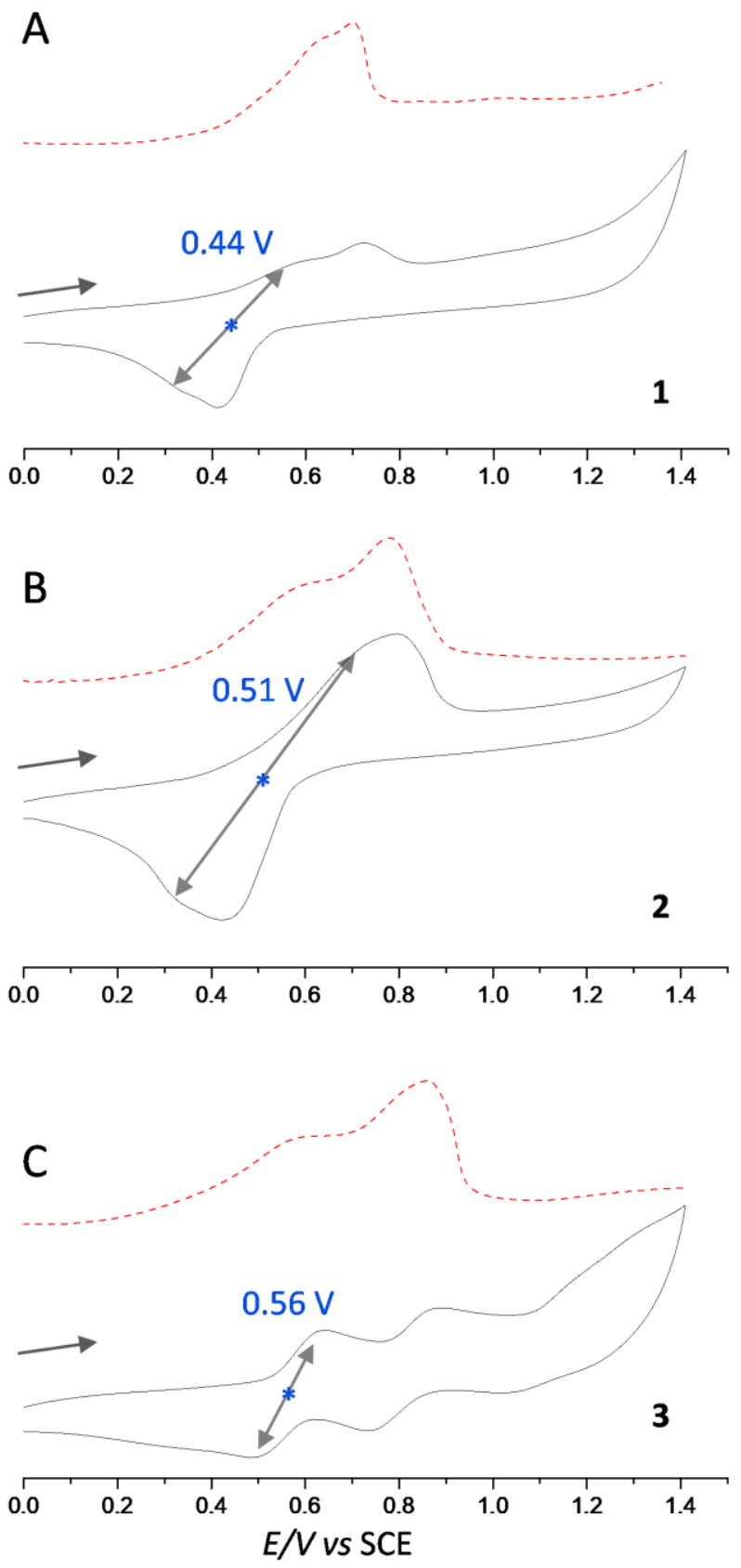

Figure S17. CV and DPV (red, dashed) diagrams of 1-3 (ca. $0.5 \mathrm{mM}$ in DCM). Supporting electrolyte: $0.1 \mathrm{M}$ $\mathrm{TBAPF}_{6}$; working electrode: Pt disk; counter electrode: Pt wire; reference electrode: $\mathrm{Ag} / \mathrm{AgNO}_{3}$; scan rate: $100 \mathrm{mV} / \mathrm{s}$. The first redox wave (blue star) of $\mathbf{1 - 3}$ was $0.44,0.51$, and $0.56 \mathrm{~V}$, respectively. DPV data suggests that both $\mathbf{2}$ and $\mathbf{3}$ have two redox processes, but the DPV peaks of $\mathbf{1}$ were not well separated. CV measurement of $\mathbf{1}$ at different scan rates suggests a pseudo-reversible electrochemical redox process (data not shown). 


\section{Chiral resolution}
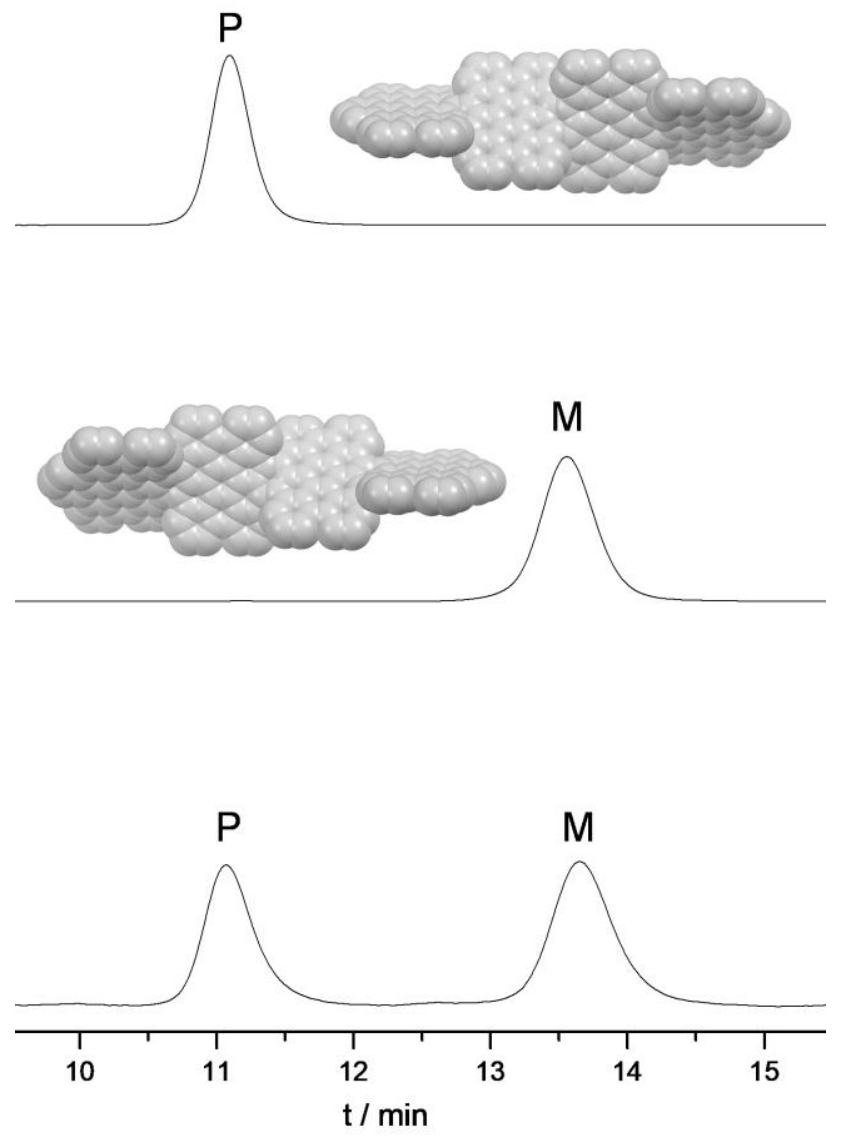

\begin{tabular}{llll}
\hline & $\mathrm{t} / \mathrm{min}$ & Area & Area $\%$ \\
\hline First & 11.07 & 148725 & 47.3 \\
Second & 13.65 & 165603 & 52.7 \\
\hline
\end{tabular}

Figure S18. Chiral HPLC traces of 1. Resolution of 1 was conducted at RT on a Shimadzu Essentia LC-16 instrument equipped with a semi-preparative COSMOSIL cholester column $(250 \times 10 \mathrm{~mm}$ i.d). Eluent: DCM/isopropanol = 3:7, v/v; flow rate: $2.0 \mathrm{~mL} / \mathrm{min}$; detected by absorption at $570 \mathrm{~nm}$. Analysis of the HPLC data (table at the bottom) suggests the presence of ca. $5 \%$ of unidentified impurities, which have the same eluting time of $(M)-\mathbf{1}$. 

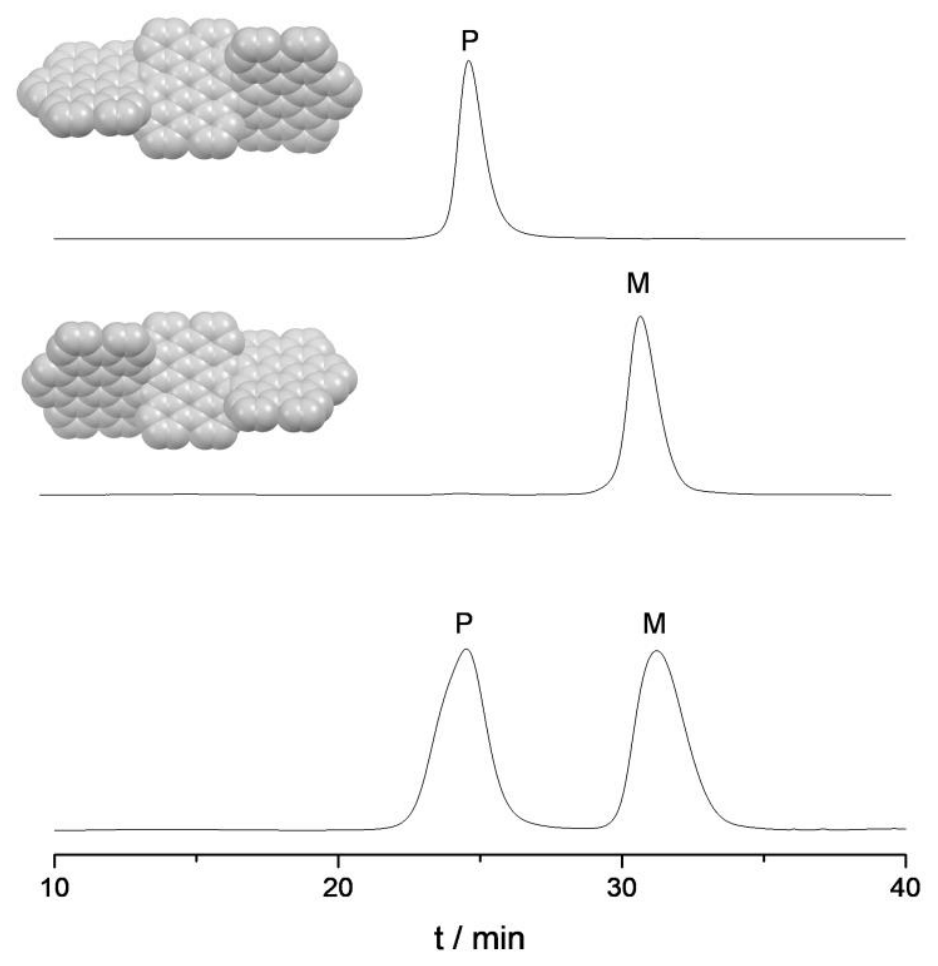

\begin{tabular}{llll}
\hline & $\mathrm{t} / \mathrm{min}$ & Area & Area $\%$ \\
\hline First & 24.52 & 32429818 & 50.1 \\
Second & 31.52 & 32314862 & 49.9 \\
\hline
\end{tabular}

Figure S19. Chiral HPLC traces of 2. Resolution of $\mathbf{2}$ was conducted at RT on a Shimadzu Essentia LC-16 instrument equipped with a semi-preparative COSMOSIL cholester column $(250 \times 10 \mathrm{~mm}$ i.d). Eluent: DCM/isopropanol = 12:10, v/v; flow rate: $2.2 \mathrm{~mL} / \mathrm{min}$; detected by absorption at $575 \mathrm{~nm}$. 

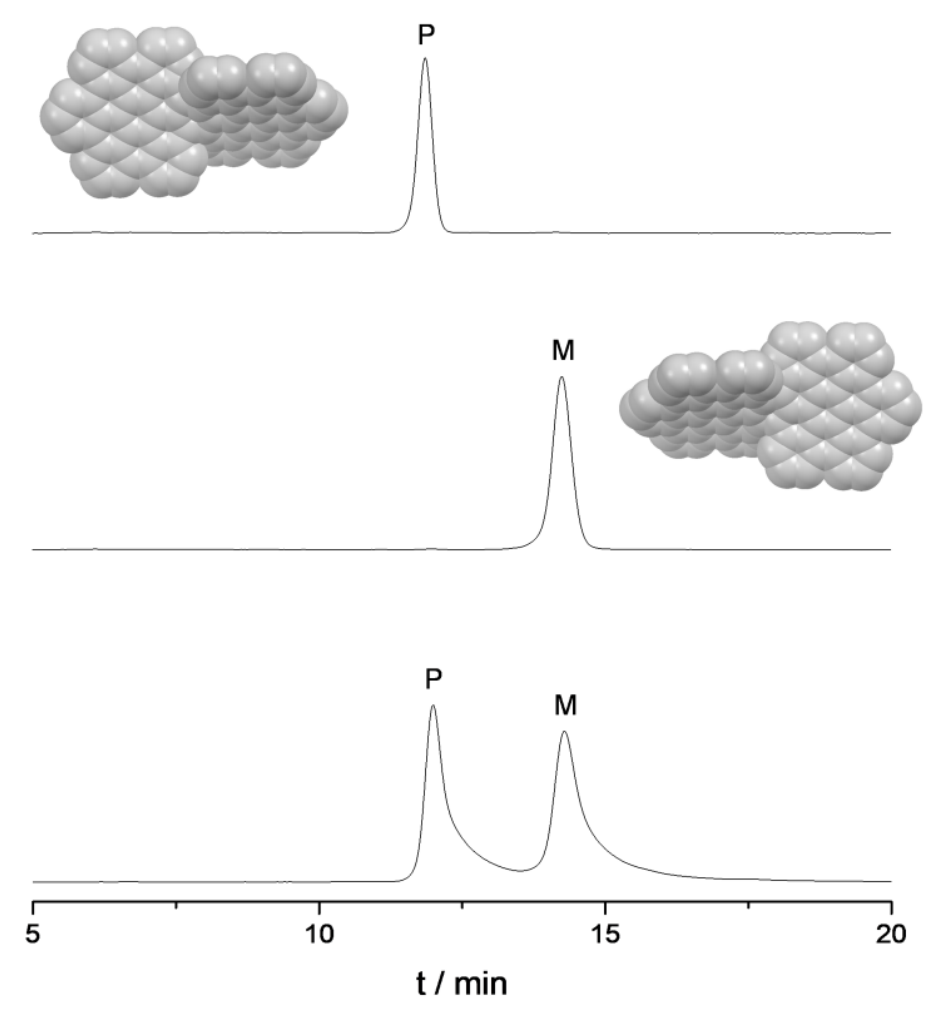

\begin{tabular}{llll}
\hline & $\mathrm{t} / \mathrm{min}$ & Area & Area $\%$ \\
\hline First & 11.99 & 5306219 & 51.3 \\
Second & 14.28 & 5044785 & 48.7 \\
\hline
\end{tabular}

Figure S20. Chiral HPLC traces of $\mathbf{3}$. Resolution of $\mathbf{3}$ was conducted at RT on a Shimadzu Essentia LC-16 instrument equipped with a semi-preparative COSMOSIL cholester column $(250 \times 10 \mathrm{~mm}$ i.d). Eluent: $\mathrm{DCM} /$ isopropanol = 3:7, v/v; flow rate: $2.0 \mathrm{~mL} / \mathrm{min}$; detected by absorption at $550 \mathrm{~nm}$. 


\section{Thermal stability}

\section{Thermal isomerization of Helical-1 and Mixing-1 at $260{ }^{\circ} \mathrm{C}$}

To examine the thermal stability of $\mathbf{1}$, we heated Helical-1 or Mixing-1 in diphenyl ether at 260 ${ }^{\circ} \mathrm{C}$ for $40 \mathrm{~h}$. Formation of other isomers was confirmed by ${ }^{1} \mathrm{H}-\mathrm{NMR}$ measurements. A visualized image of thin layer chromatography (TLC) as well as the chiral HPLC data were shown below:

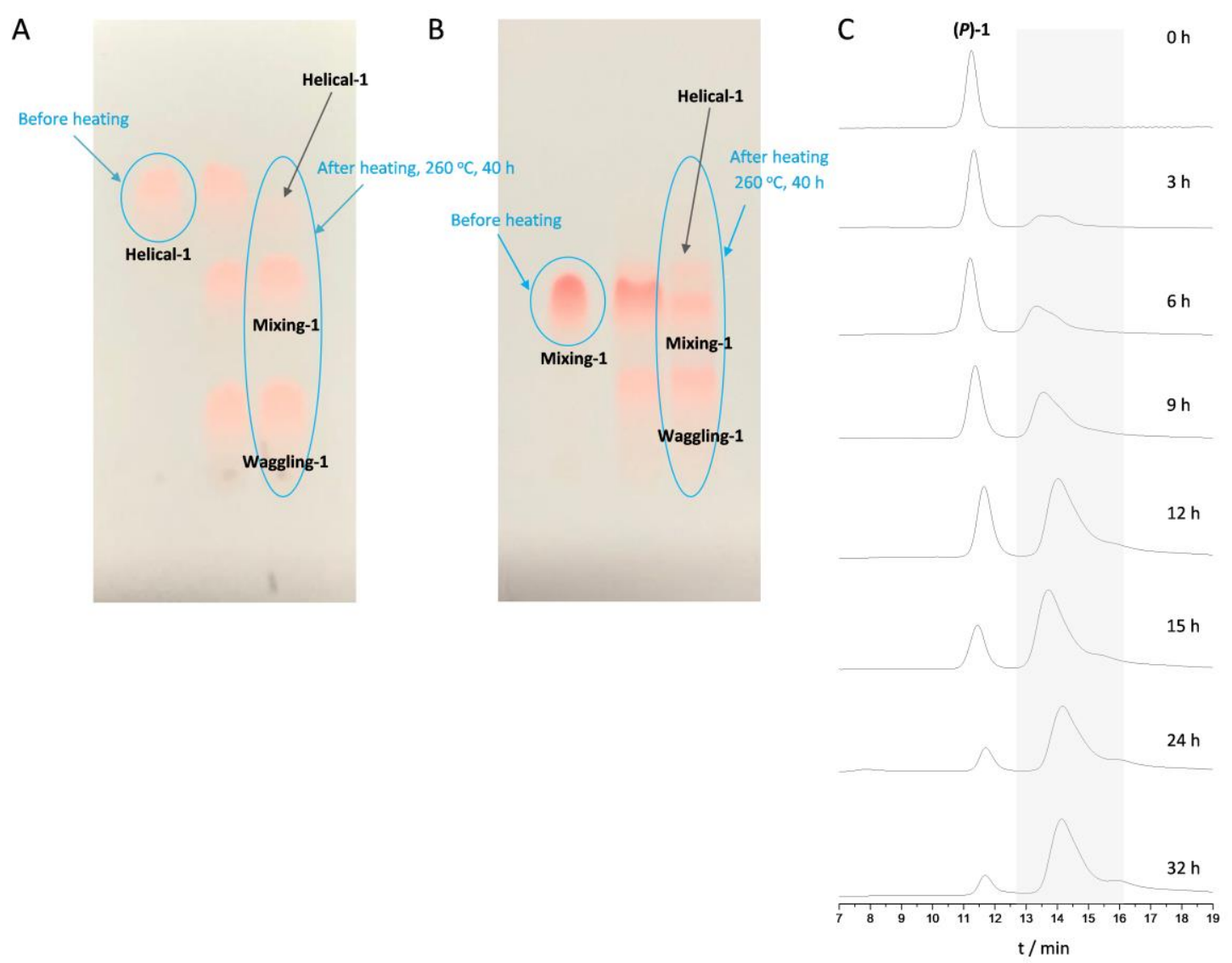

Figure S21. Thermal isomerization of (A) Helical-1 and (B) Mixing-1 to other isomers followed by silica gel coated TLC (eluent: PE/DCM, v/v, 3/1), after heating in diphenyl ether at $260{ }^{\circ} \mathrm{C}$ for $40 \mathrm{~h}$. (C) Chiral HPLC traces following the isomerization of $(P)-\mathbf{1}$ upon heating at $260^{\circ} \mathrm{C}$ for $32 \mathrm{~h}$. DFT calculations, at the B3LYP/6$31 \mathrm{G}(\mathrm{d})$ level of theory, indicate that Waggling-1 (-8.1 kJ/mol) is more stable compared to Mixed-1 (-4.5 $\mathrm{kJ} / \mathrm{mol})$ and Helical-1 $(0 \mathrm{~kJ} / \mathrm{mol})$. These theoretical data suggest an equilibrium constant $K$ of 2.2, 2.8 and 6.1, respectively, between three different isomers, being qualitatively consistent with the HPLC and TLC analysis shown above. 


\section{Thermal isomerization of $(P)-3$ at $260{ }^{\circ} \mathrm{C}$}

Thermal isomerization of $\mathbf{3}$ was investigated readily by chiral HPLC. It switches between the $(P)$ and $(M)$-form isomers following a reversible first order reaction, ${ }^{2}$ with a rate constant $(k)$, which could be obtained by fitting the experimental data $(\alpha$, mole ratio of $(P)-\mathbf{3}$ at time $t)$ using the following equation:

$$
\ln (2 \alpha-1)=-2 k t
$$

Then the isomerization barrier $\left(\Delta \mathrm{G}^{\ddagger}\right)$ was calculated from the Eyring equation:

$\Delta G^{\ddagger}(T)=-R T \ln \left(k h / \kappa k_{B} T\right)$

$R$ is the gas constant $\left(R=8.31441 \mathrm{~J} \mathrm{~K}^{-1}\right) ; h$ is the Planck constant $\left(h=6.626176 \times 10^{-34} \mathrm{~J} \mathrm{~s}\right) ; k_{B}$ is the Boltzmann constant $\left(k_{B}=\right.$ $\left.1.380662 \times 10^{-23} \mathrm{~J} \mathrm{~K}^{-1}\right) ; \kappa$ is the transmission coefficient $(\kappa=1)$.

A

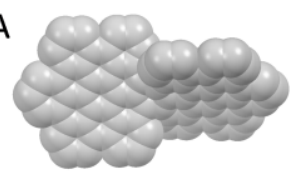

$(P)-3$
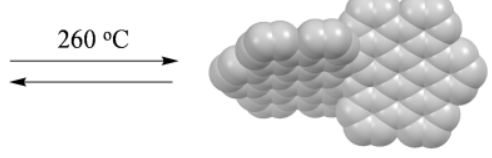

(M)-3
B $\quad \alpha$ : mole ratio of $(P)-3$

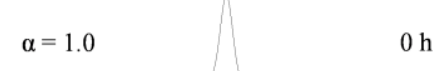

(P)-3

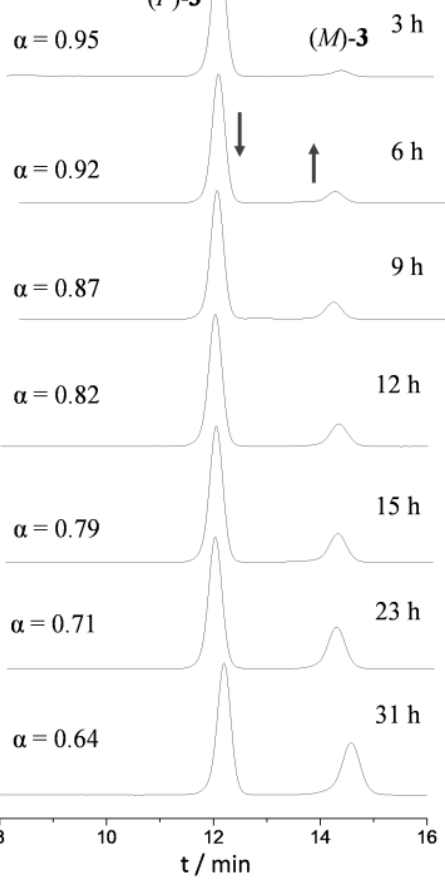

Figure S22. (A) Thermal isomerization of $(P)-3$ in diphenyl ether at $260{ }^{\circ} \mathrm{C}$ monitored by (B) chiral HPLC with a semi-preparative COSMOSIL cholester column $(250 \times 10 \mathrm{~mm}$ i.d $)$. Eluent: DCM/isopropanol = 3:7, $\mathrm{v} / \mathrm{v}$; flow rate: $2.0 \mathrm{~mL} / \mathrm{min}$; detected by absorption at $550 \mathrm{~nm}$. 
8. NMR and mass spectra (Note: Due to limited solubility and similar chemical environments, ${ }^{13} \mathrm{C}-\mathrm{NMR}$ spectra of several compounds, e.g. 9, 10, and 1, are not good, even dissolved in mixed solvent $\left(\mathrm{CS}_{2} / \mathrm{CDCl}_{3}\right)$ with prolonged data collection up to $24 \mathrm{~h}$. Overlapped ${ }^{13} \mathrm{C}-\mathrm{NMR}$ signals were observed frequently, see Table S9 )

Table S9. Statistics on the number of ${ }^{13} \mathrm{C}-\mathrm{NMR}$ signals

\begin{tabular}{ccc}
\hline Compounds & $\begin{array}{c}\text { Number of Aromatic }{ }^{13} \mathrm{C} \\
\text { (expected/observed) }\end{array}$ & $\begin{array}{c}\text { Number of Aliphatic }{ }^{13} \mathrm{C} \\
\text { (expected/observed) }\end{array}$ \\
\hline $\mathbf{1}$ & $42 / 28$ & $10 / 8$ \\
$\mathbf{2}$ & $32 / 22$ & $8 / 6$ \\
$\mathbf{3}$ & $22 / 18$ & $6 / 4$ \\
$\mathbf{4}$ & $7 / 7$ & $2 / 2$ \\
$\mathbf{6}$ & $19 / 17$ & $4 / 2$ \\
$\mathbf{7}$ & $18 / 21$ & $4 / 4$ \\
$\mathbf{8}$ & $18 / 21$ & $4 / 4$ \\
$\mathbf{9}$ & $22 / 18$ & $4 / 4$ \\
$\mathbf{1 1}$ & $38 / 30$ & $10 / 8$ \\
$\mathbf{1 7}$ & $7 / 7$ & $2 / 2$ \\
$\mathbf{1 8}$ & $18 / 18$ & $4 / 4$ \\
\hline
\end{tabular}

Note: Compound 10 shows an extremely poor solubility, its ${ }^{13} \mathrm{C}-\mathrm{NMR}$ data was not available. Compounds 12-16 are intermediates for the synthesis of $\mathbf{2}$. Their NMR data could be found in Campaña's paper (Angew. Chem., Int. Ed. 2020, 59, 7139-7145). 
${ }^{1} \mathrm{H}$ NMR and ${ }^{13} \mathrm{C}$ NMR spectra of 4

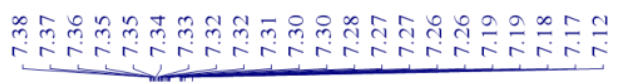

$\sqrt[n]{n-m}$
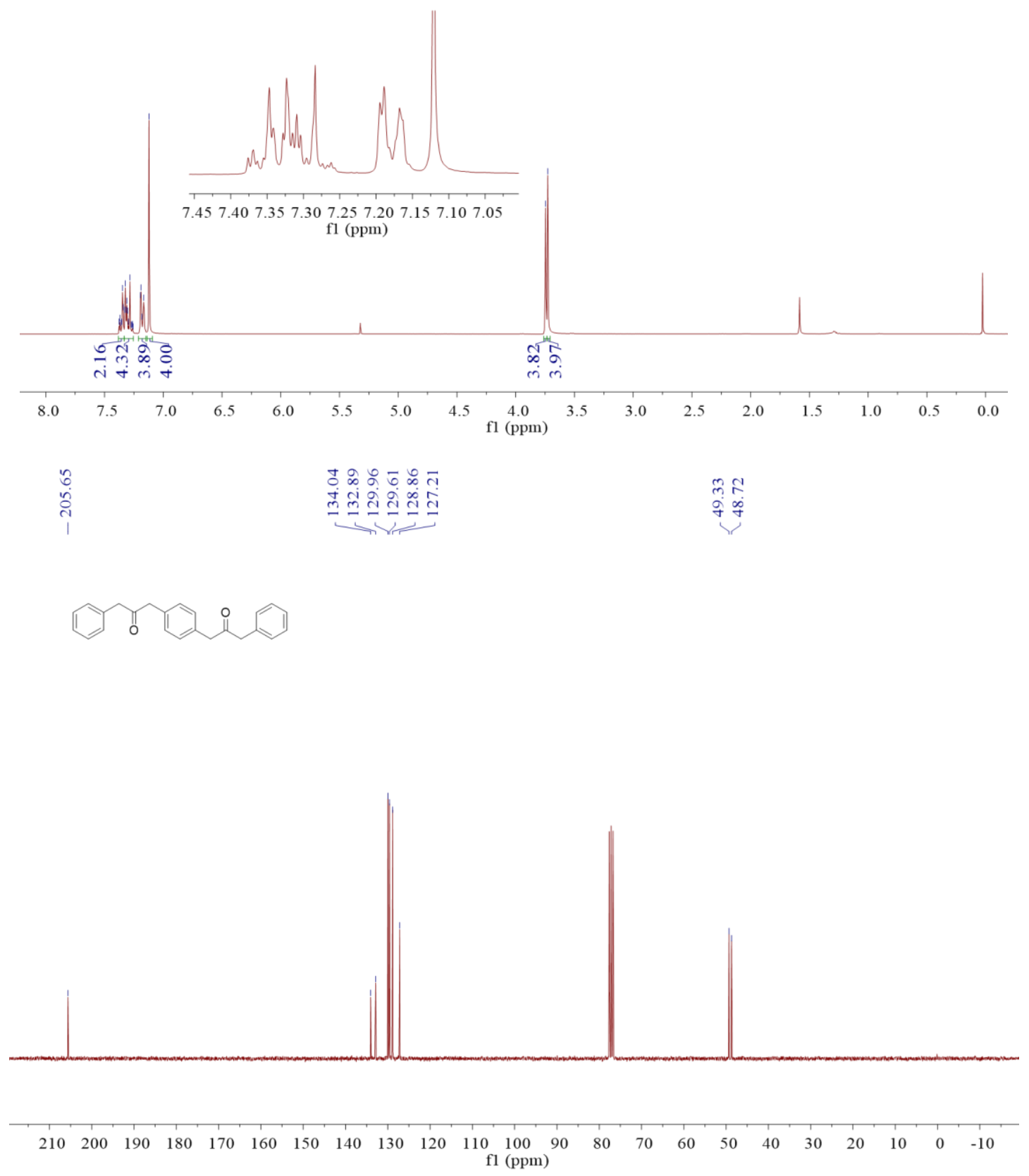

S51 


\section{${ }^{1} \mathrm{H}$ NMR and ${ }^{13} \mathrm{C}$ NMR spectra of 6}

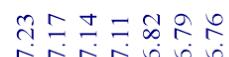

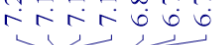
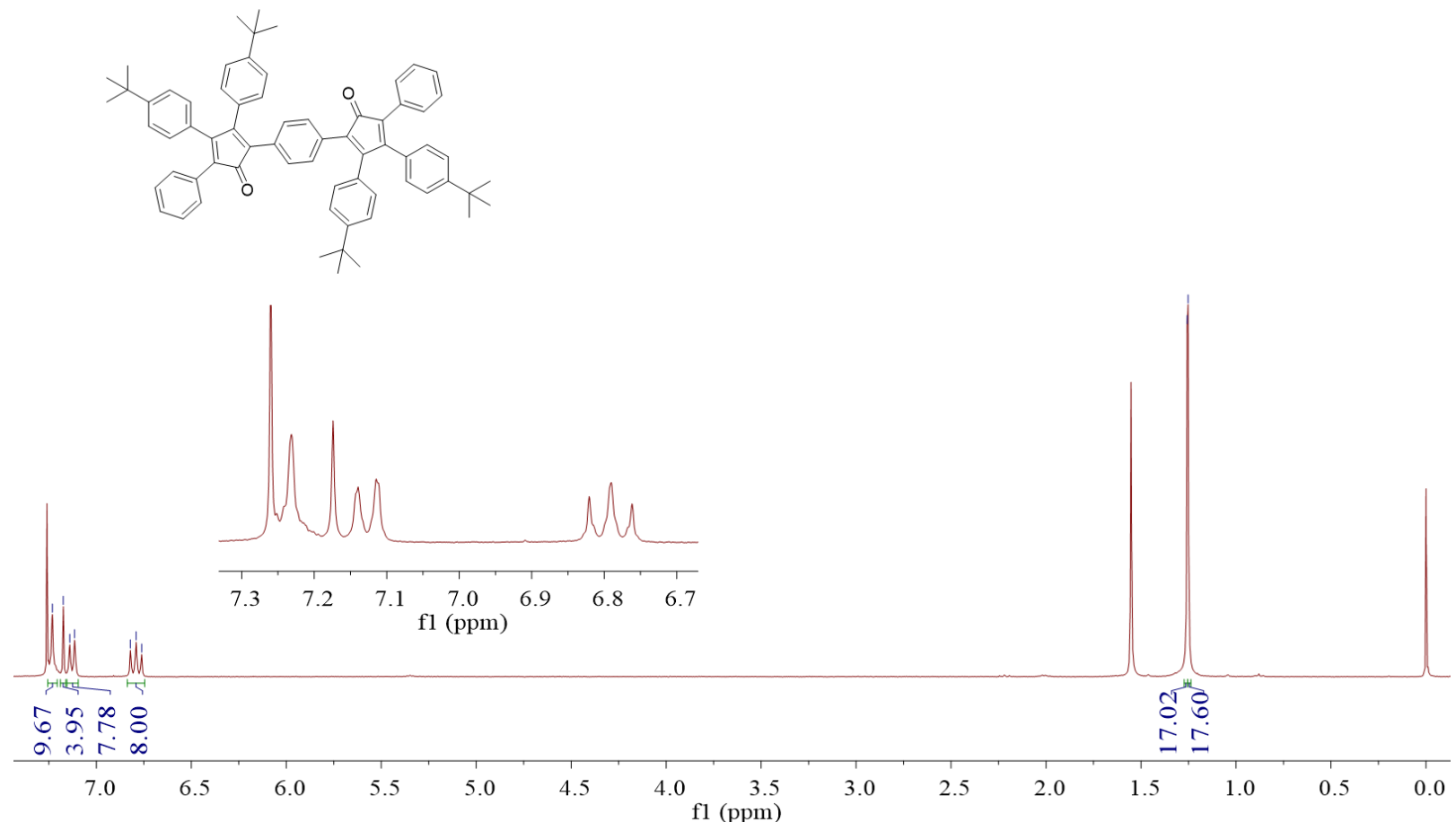

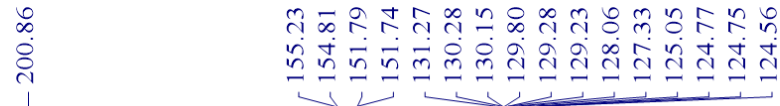

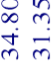
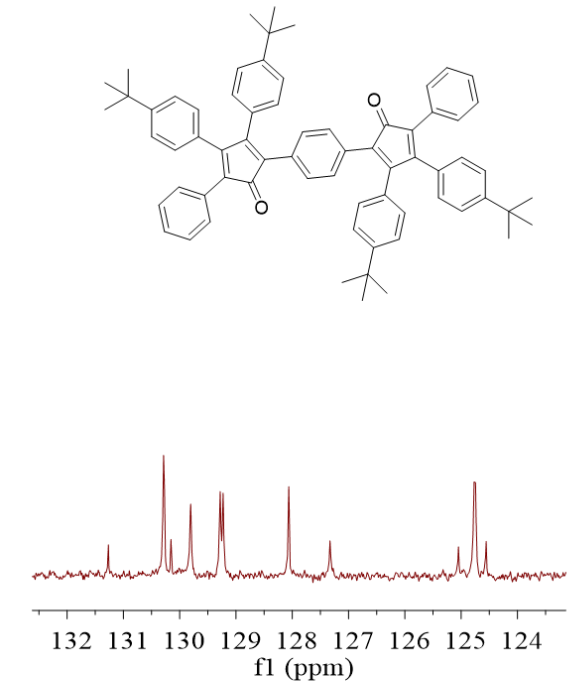

f1 (ppm)

$\begin{array}{llllllllllllllllllllllllll}210 & 200 & 190 & 180 & 170 & 160 & 150 & 140 & 130 & 120 & 110 & 100 & 90 & 80 & 70 & 60 & 50 & 40 & 30 & 20 & 10 & 0 & -10\end{array}$ fl (ppm) 


\section{${ }^{1} \mathrm{H}$ NMR and ${ }^{13} \mathrm{C}$ NMR spectra of 7}

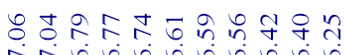

r.

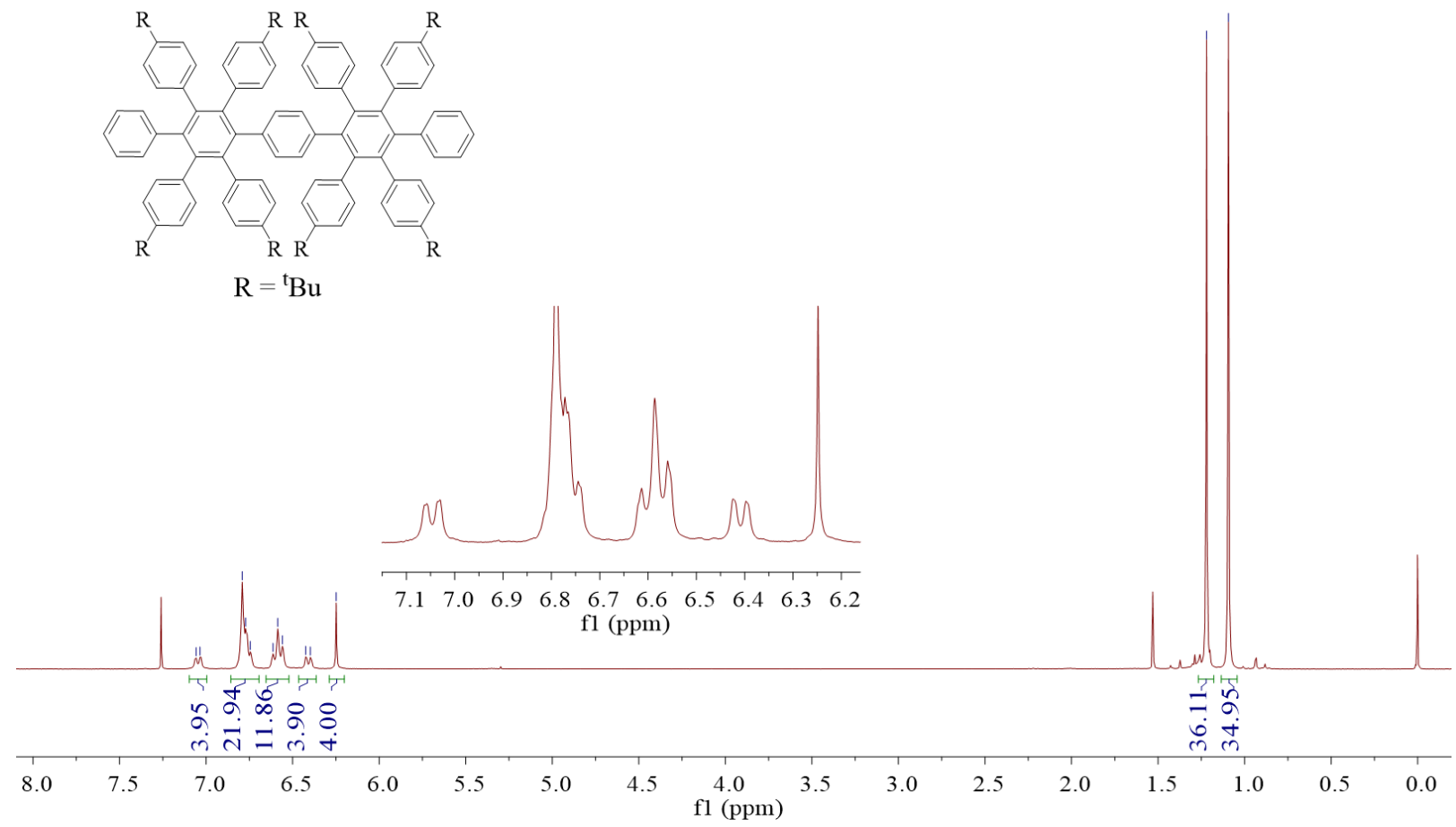

$\mathrm{R}={ }^{\mathrm{t}} \mathrm{Bu}$

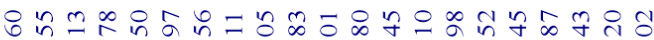

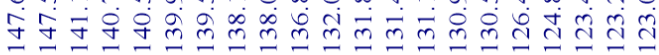

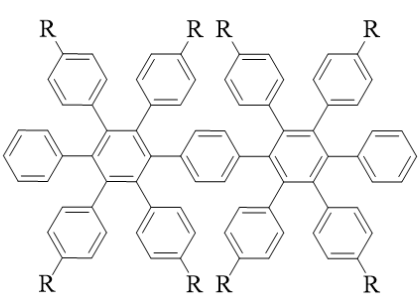

$\mathrm{R}={ }^{\mathrm{t}} \mathrm{Bu}$
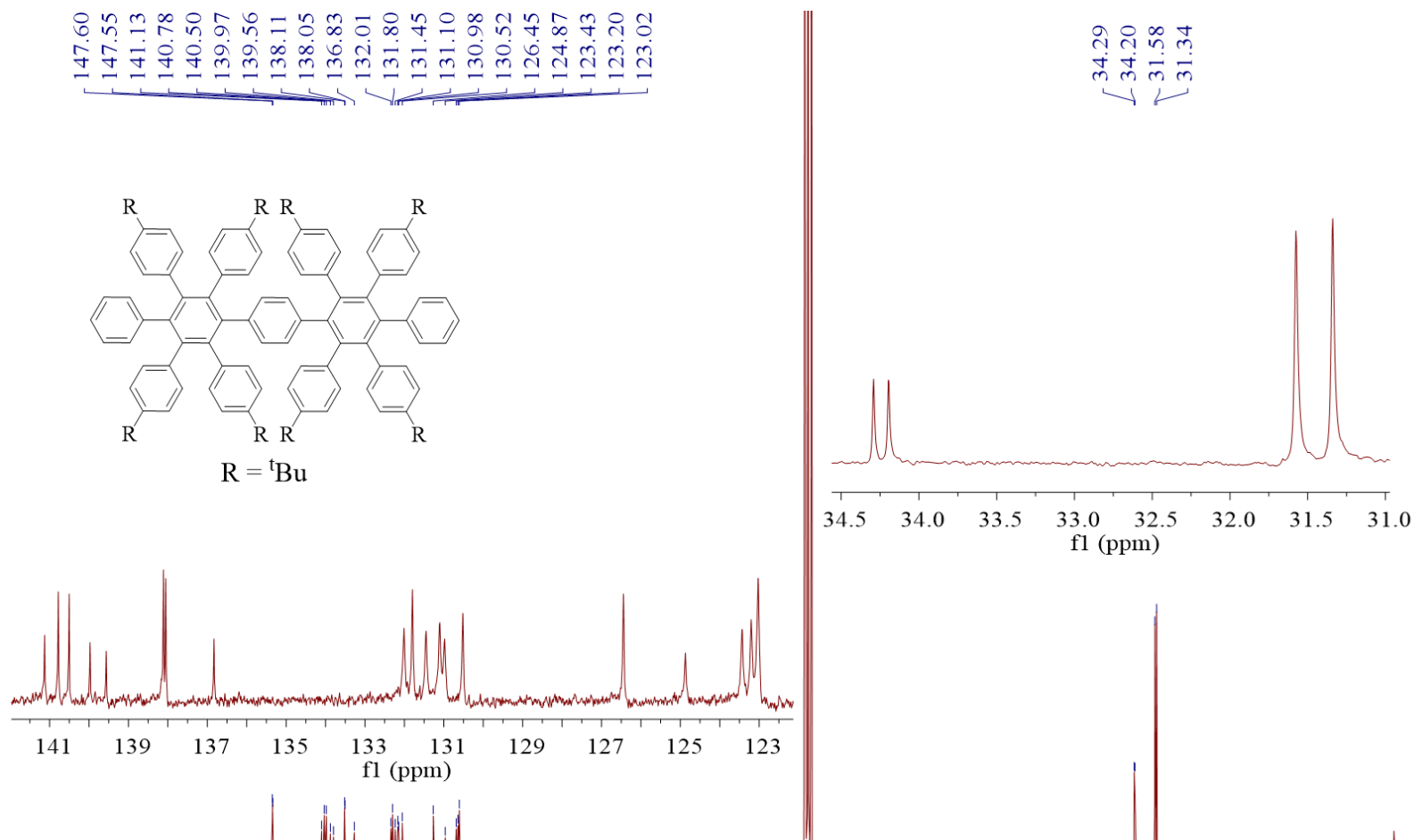

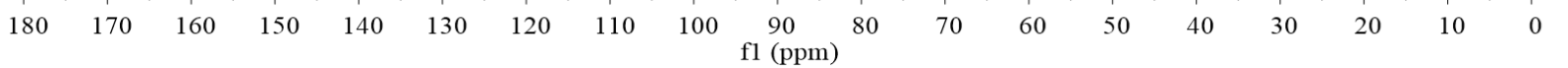




\section{${ }^{1} \mathrm{H}$ NMR and ${ }^{13} \mathrm{C}$ NMR spectra of 8}

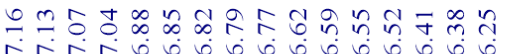
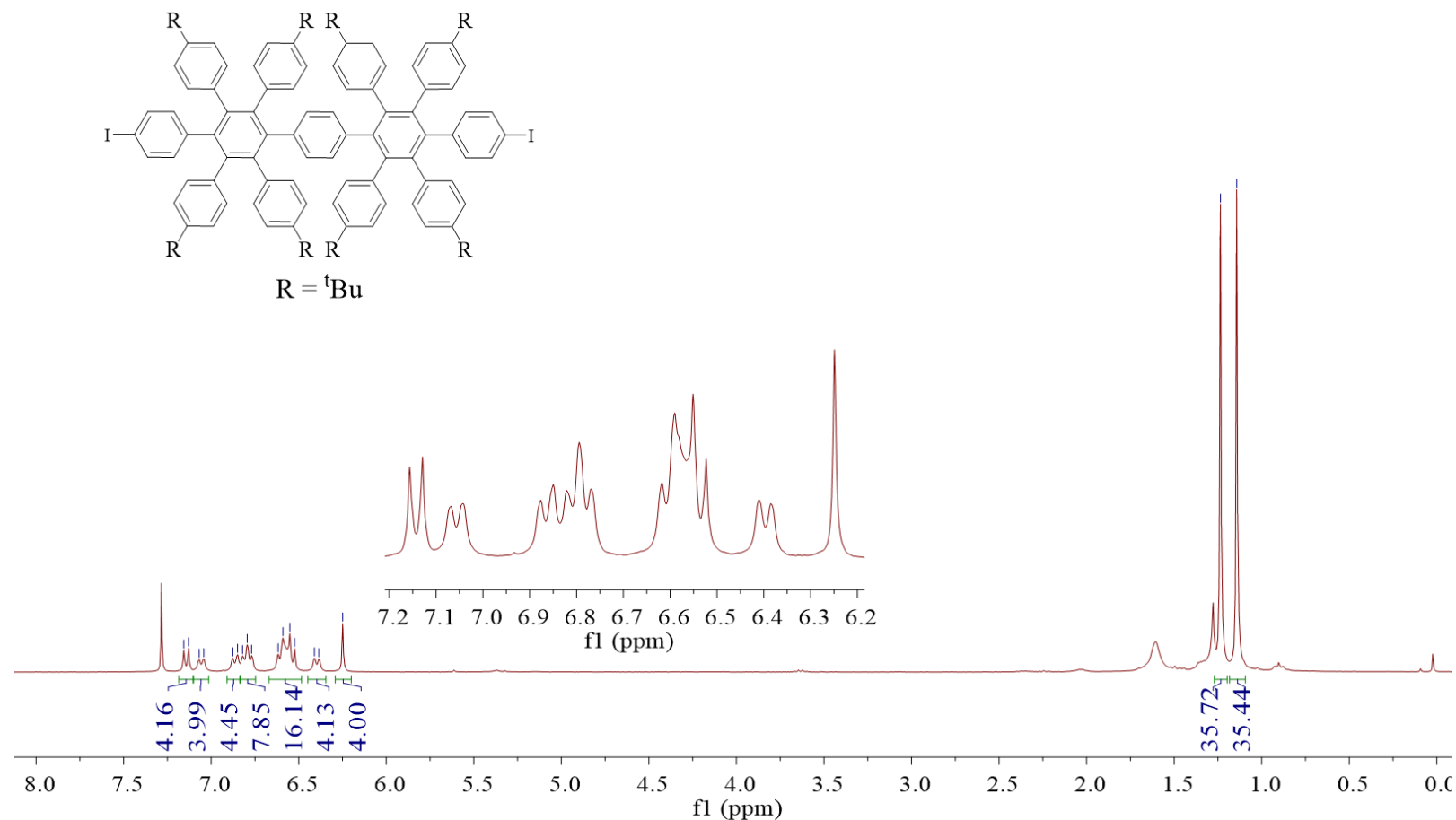

ㅇำ

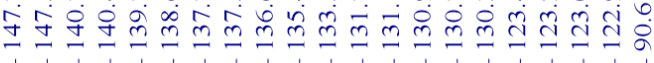

은 곡

मेंगेंल

$\checkmark r$
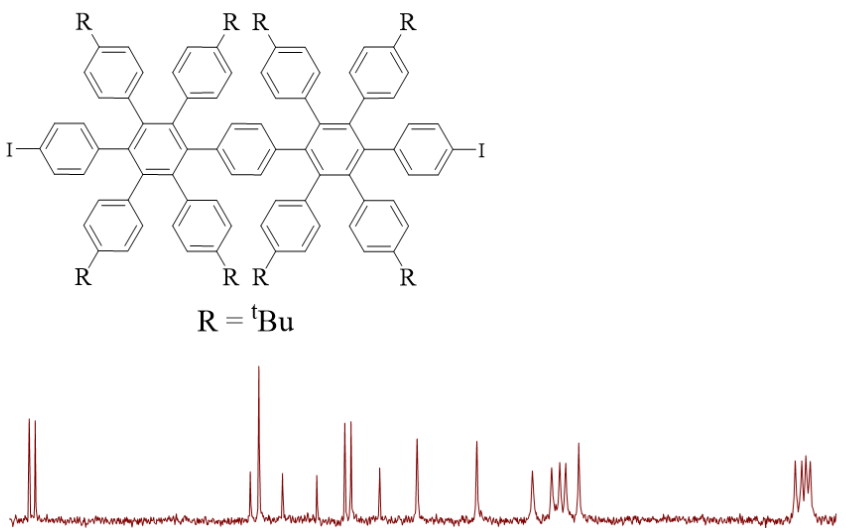

$\begin{array}{lllllllllllll}148 & 146 & 144 & 142 & 140 & 138 & 136 & 134 & 132 & 130 & 128 & 126 & 124\end{array}$ fl (ppm)

$\begin{array}{lllllllllllllllllllllllllll}210 & 200 & 190 & 180 & 170 & 160 & 150 & 140 & 130 & 120 & 110 & 100 & 90 & 80 & 70 & 60 & 50 & 40 & 30 & 20 & 10 & 0 & -10\end{array}$ fl (ppm)
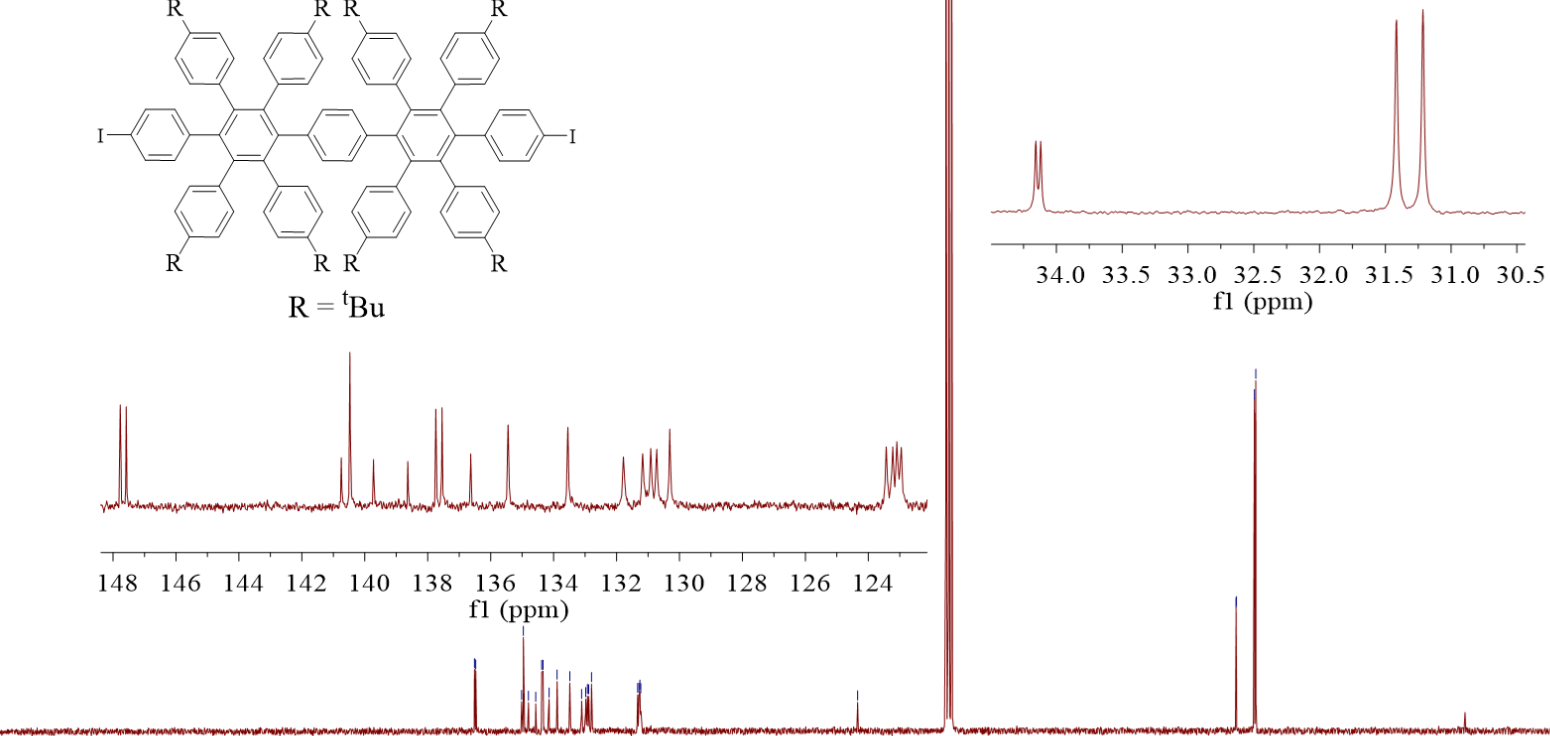
${ }^{1} \mathrm{H}$ NMR and ${ }^{13} \mathrm{C}$ NMR spectra of 9 (Note: ${ }^{13} \mathrm{C}$ NMR spectrum is not good due to limited solubility)

भे थैं

a

$\stackrel{\infty}{i} \stackrel{m}{i}$
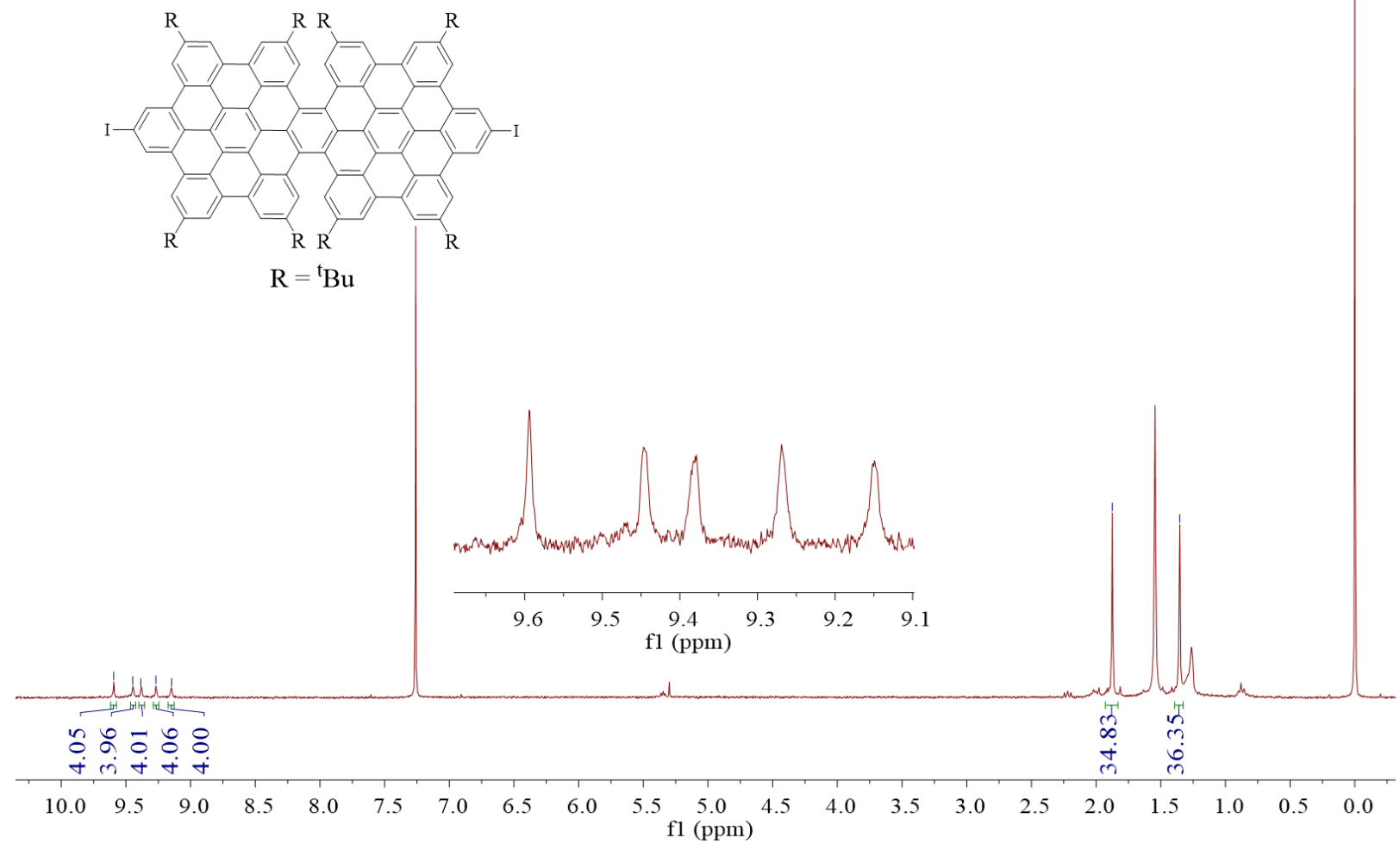

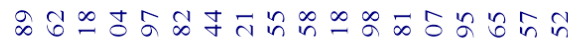

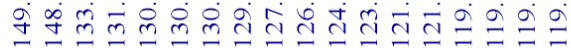

ㅇํㄹำ

क्यांक
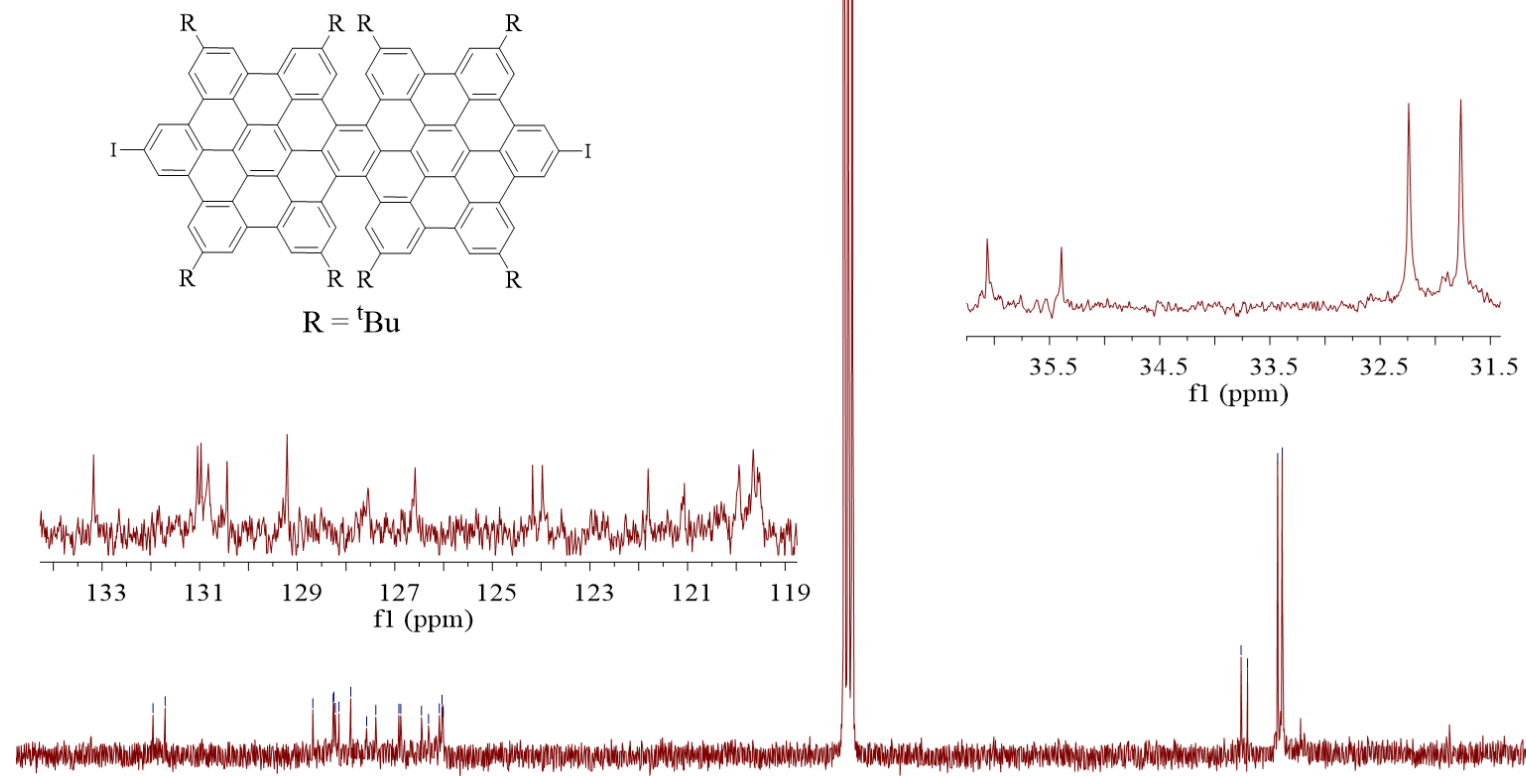

f1 $(\mathrm{ppm})$ 
${ }^{1} \mathrm{H}$ NMR spectrum of 10 (Note: We could not collect the ${ }^{13} \mathrm{C}$ NMR spectrum because of limited solubility)

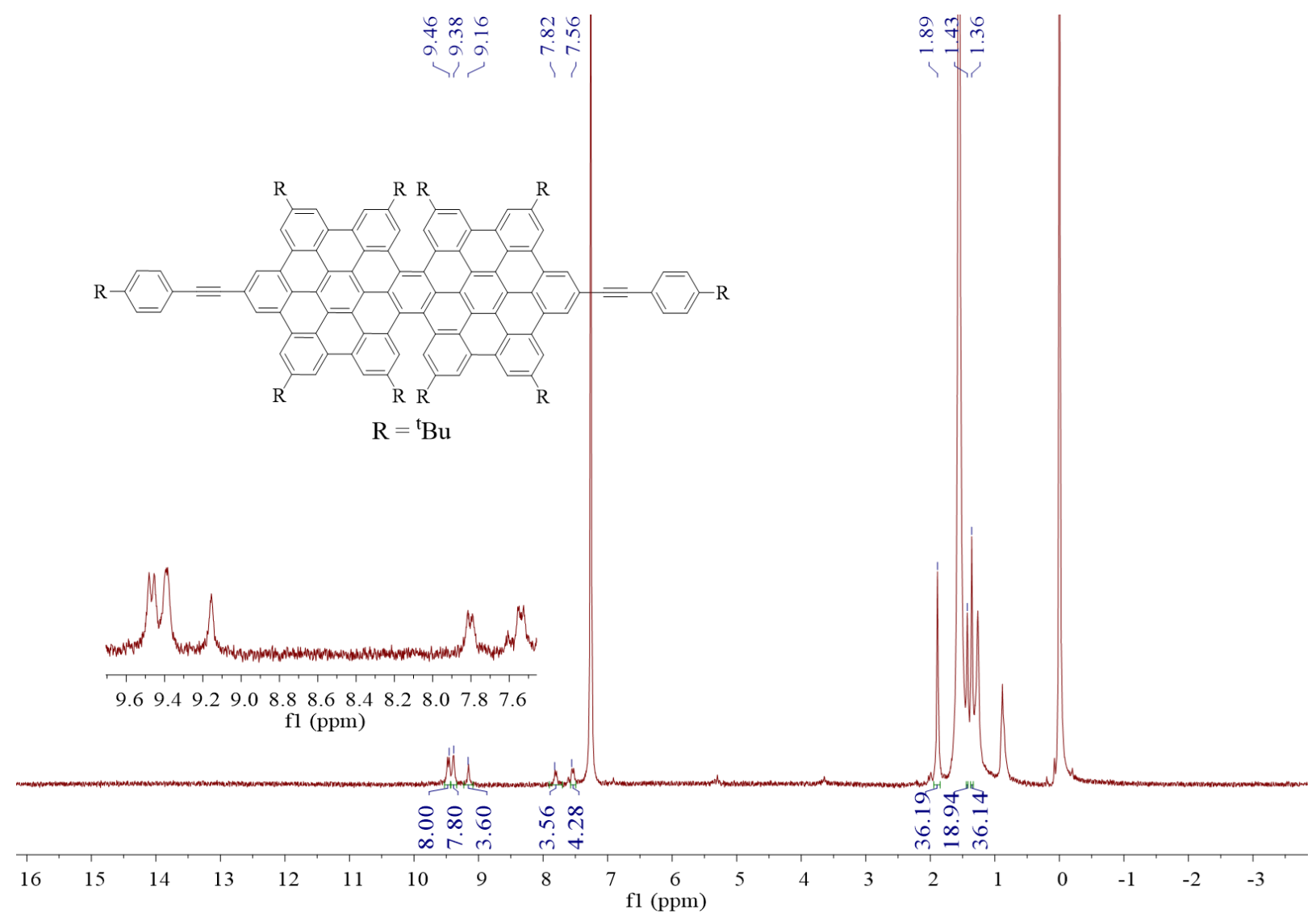




\section{${ }^{1} \mathrm{H}$ NMR and ${ }^{13} \mathrm{C}$ NMR spectra of 11}

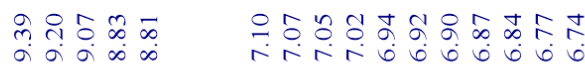

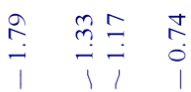

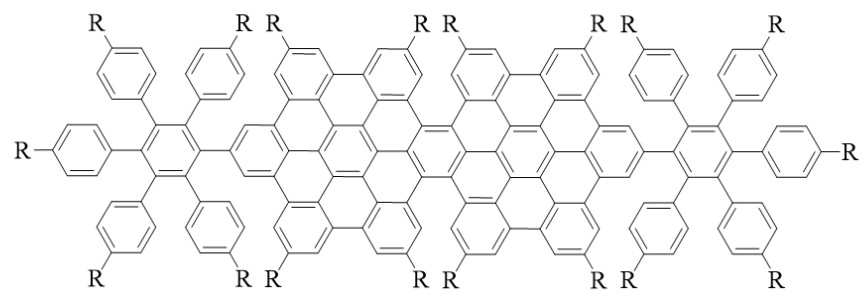

$$
\mathrm{R}={ }^{\mathrm{t}} \mathrm{Bu}
$$
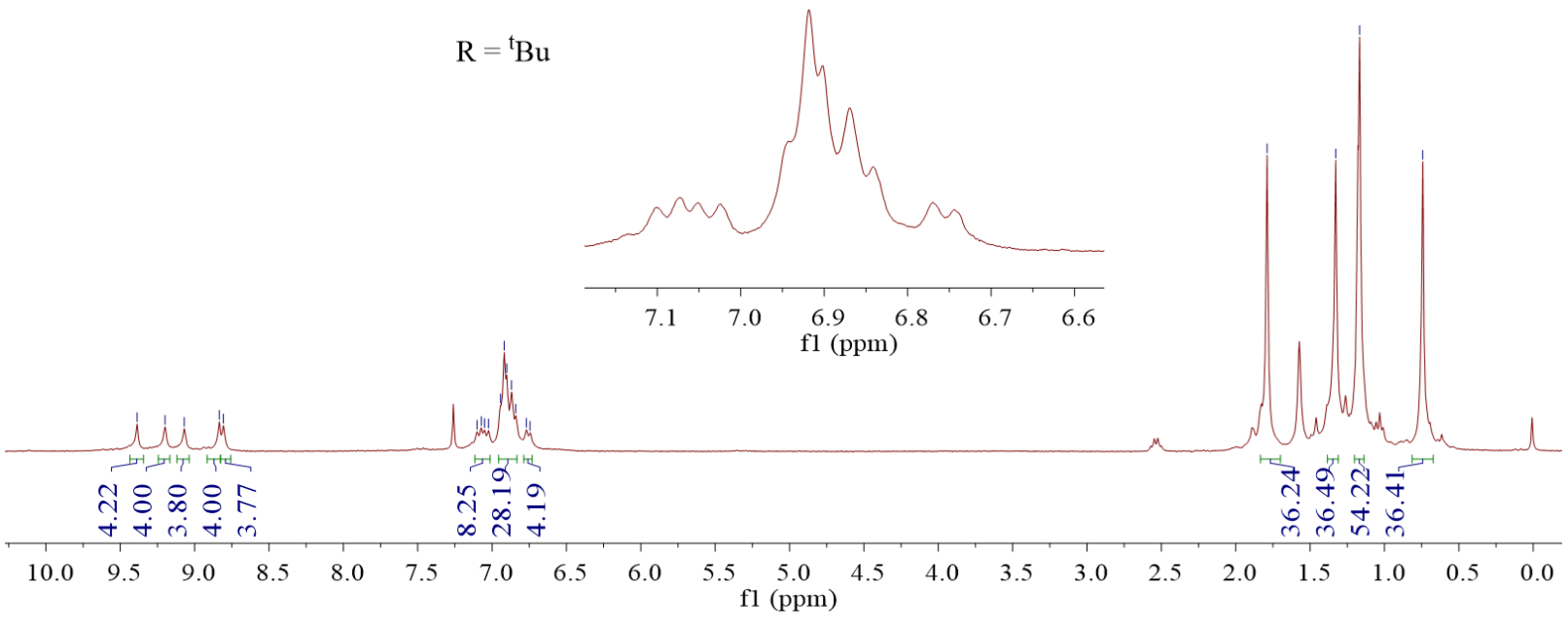

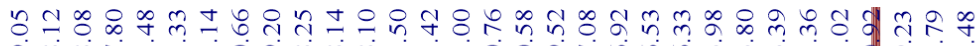

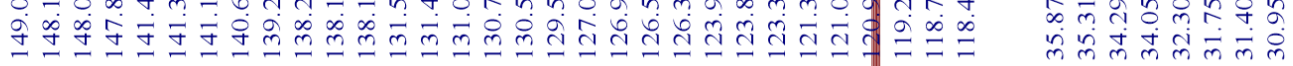

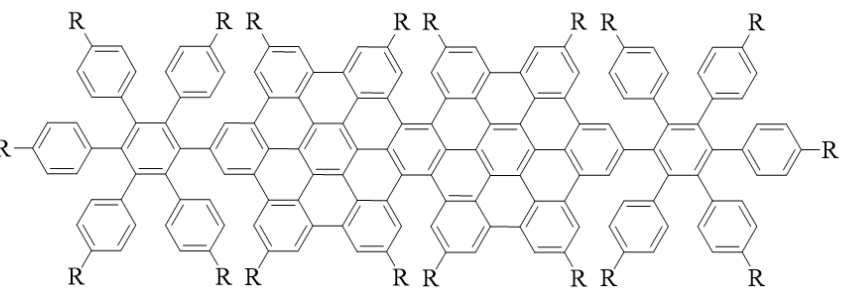

$\mathrm{R}={ }^{\mathrm{t}} \mathrm{Bu}$

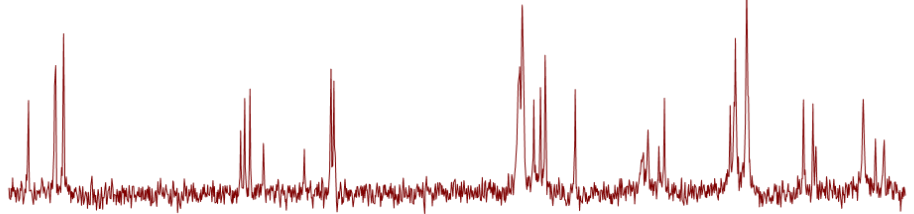

$\begin{array}{llllllllllllllll}148 & 146 & 144 & 142 & 140 & 138 & 136 & 134 & 132 & 130 & 128 & 126 & 124 & 122 & 120 & 118\end{array}$ f1 (ppm)

$\begin{array}{lllllllllllllllllllllllll}210 & 200 & 190 & 180 & 170 & 160 & 150 & 140 & 130 & 120 & 110 & 100 & 90 & 80 & 70 & 60 & 50 & 40 & 30 & 20 & 10 & 0 & -10\end{array}$ fl (ppm) 
${ }^{1} \mathrm{H}$ NMR and ${ }^{13} \mathrm{C}$ NMR spectra of 1 (Note: ${ }^{13} \mathrm{C}$ NMR spectrum is not good due to limited solubility)
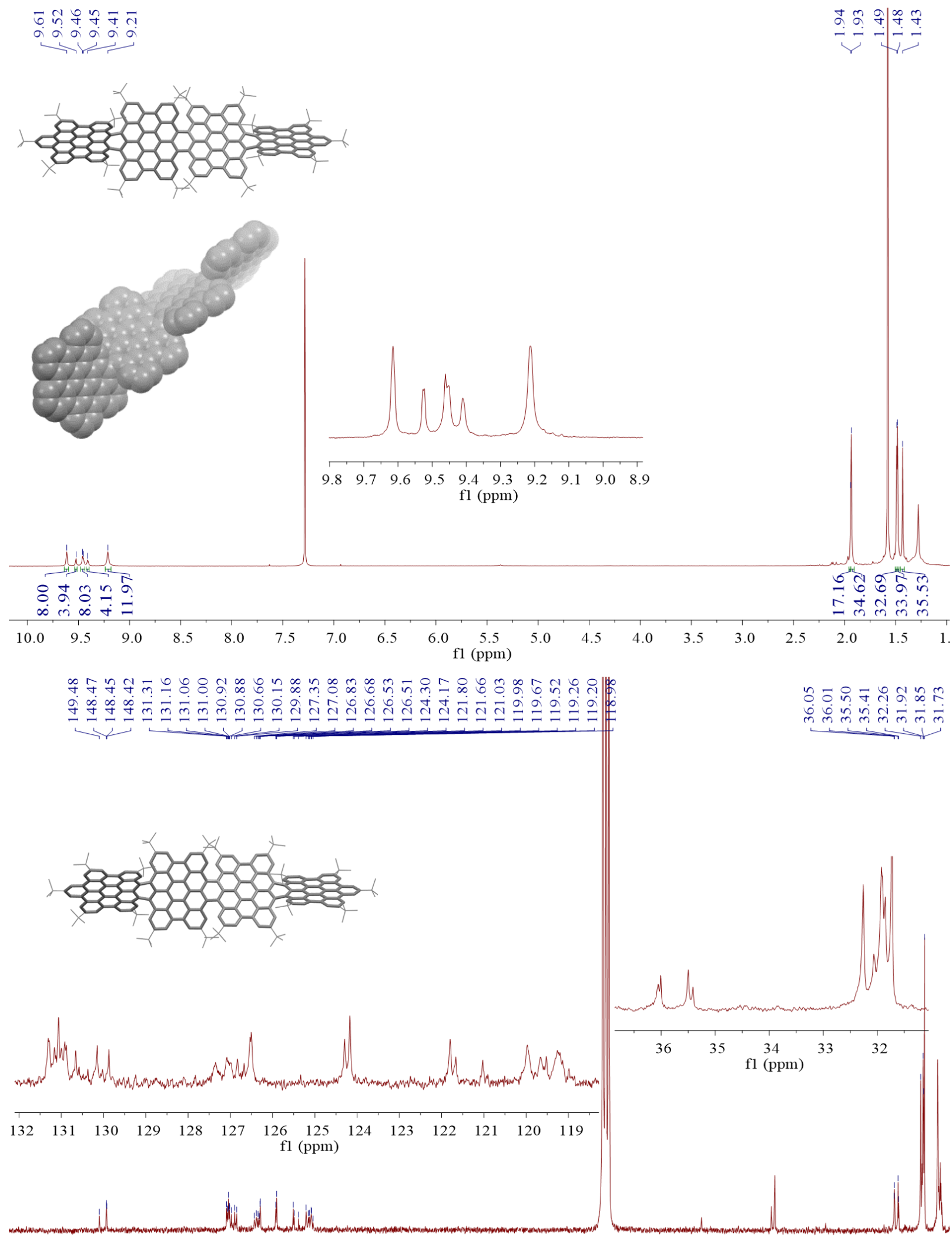

$\begin{array}{llllllllllllllllllllllllllll}160 & 155 & 150 & 145 & 140 & 135 & 130 & 125 & 120 & 115 & 110 & 105 & 100 & 95 & 90 & 85 & 80 & 75 & 70 & 65 & 60 & 55 & 50 & 45 & 40 & 35 & 30 \\ \text { f1 (pm) }\end{array}$ 


\section{${ }^{1} \mathrm{H}-{ }^{1} \mathrm{H}$ ROE spectrum of 1}

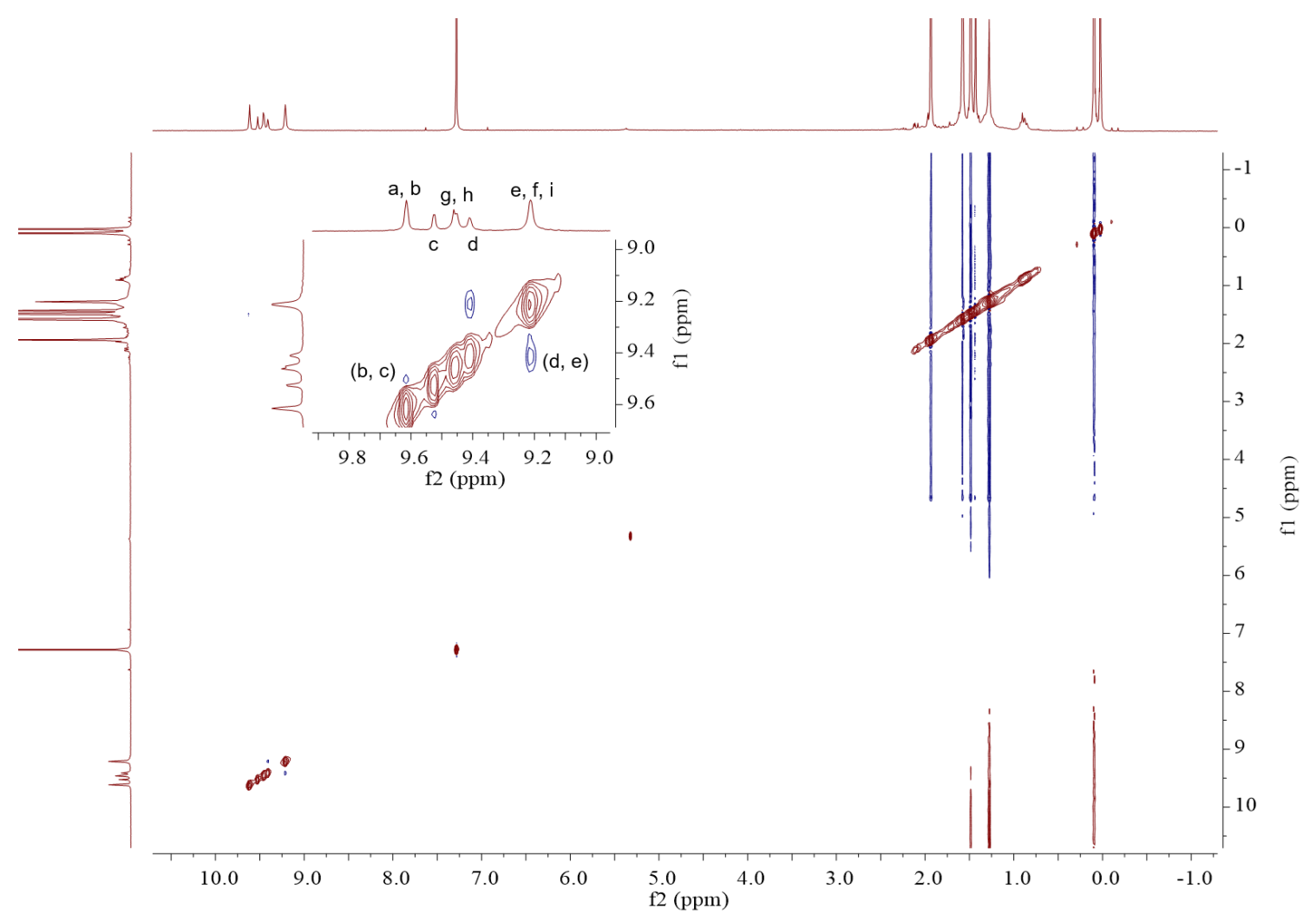

\section{${ }^{1} \mathrm{H}-{ }^{13} \mathrm{C}$ HSQC spectrum of 1}

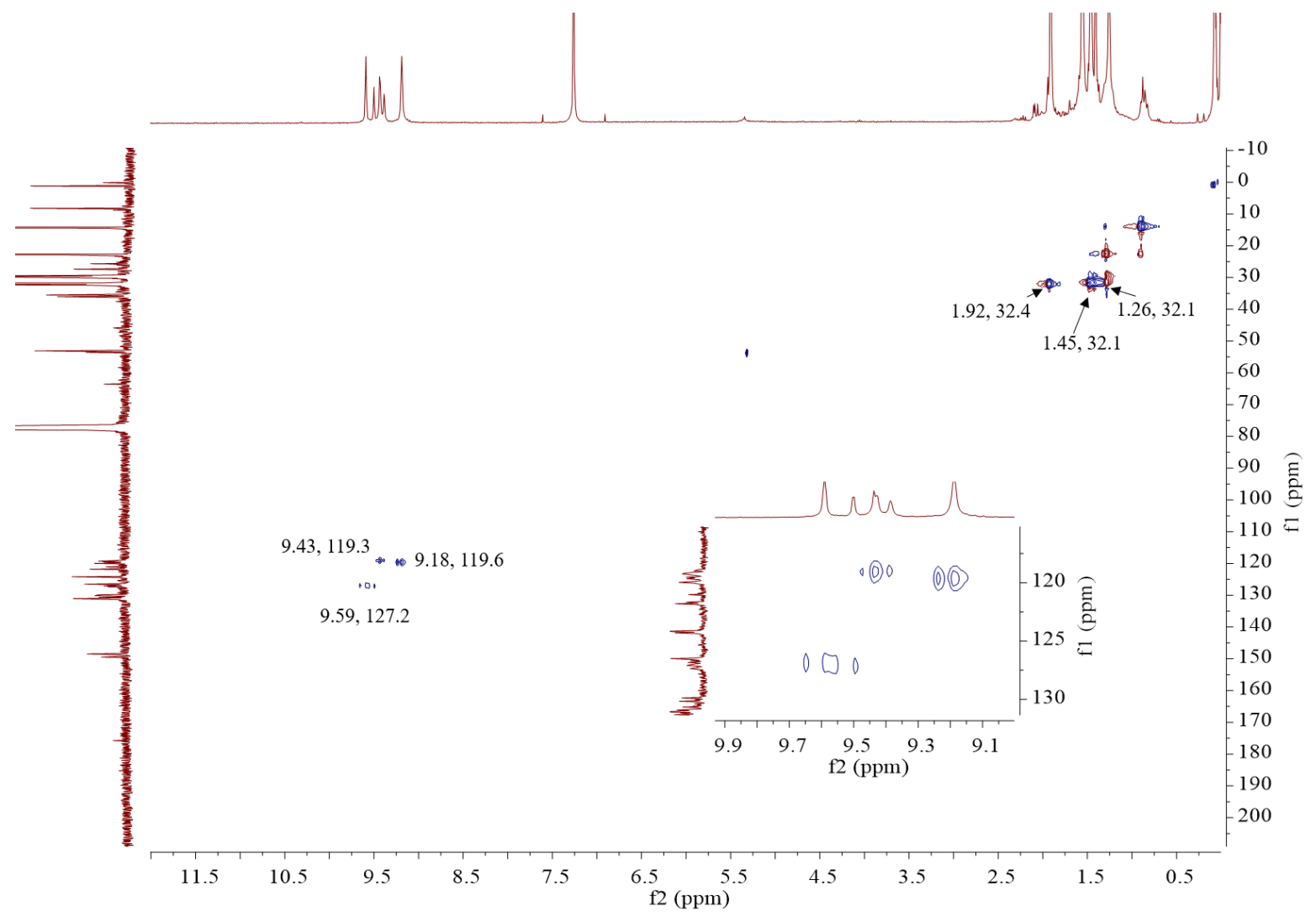


${ }^{1} \mathrm{H}-{ }^{13} \mathrm{C}$ HMBC spectrum of 1

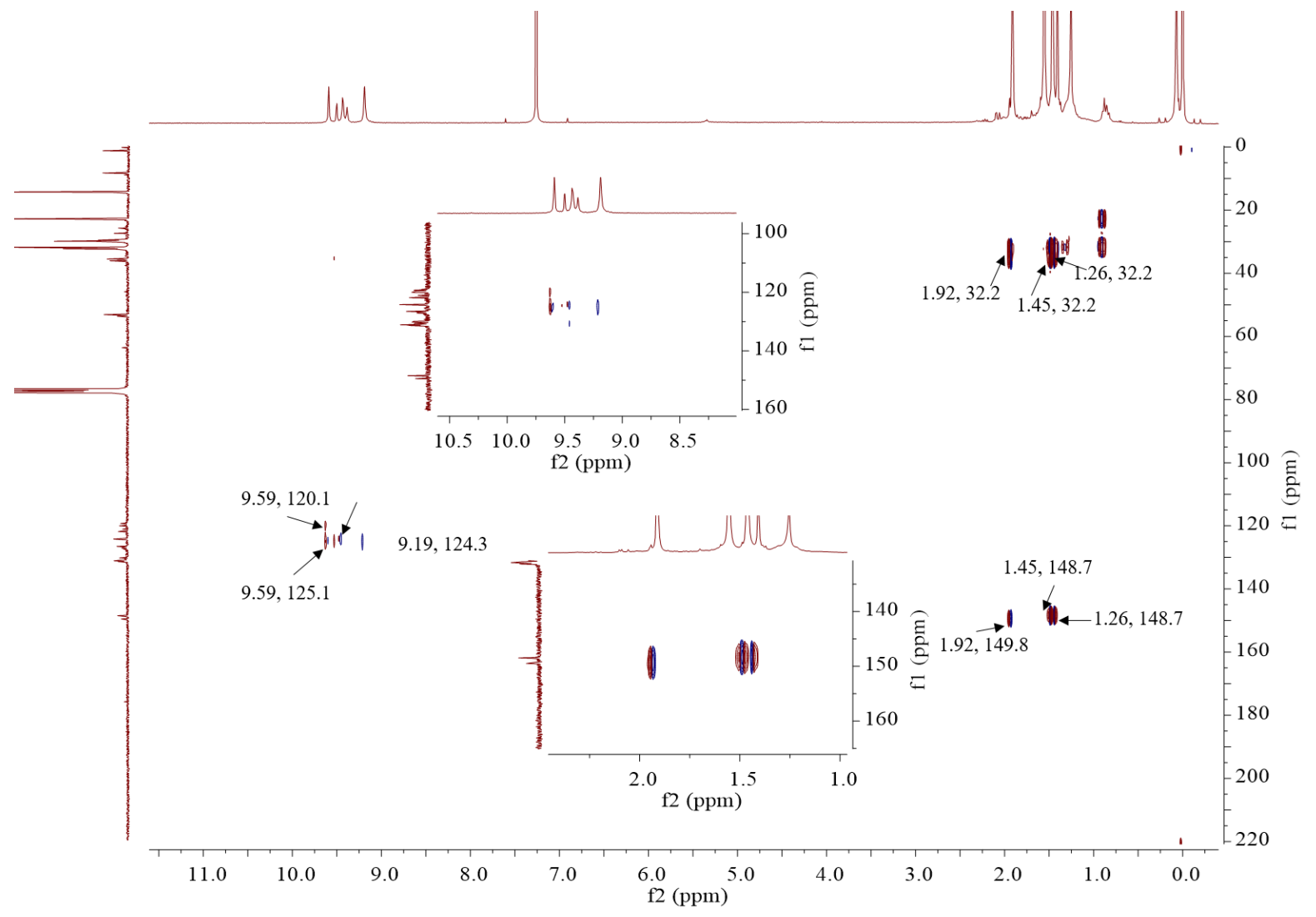


${ }^{1} \mathrm{H}$ NMR and ${ }^{13} \mathrm{C}$ NMR spectra of Mixed-1 (Note: ${ }^{13} \mathrm{C}$ NMR spectrum is not good due to

\section{limited solubility)}

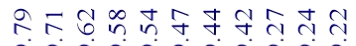

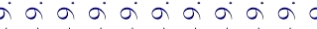
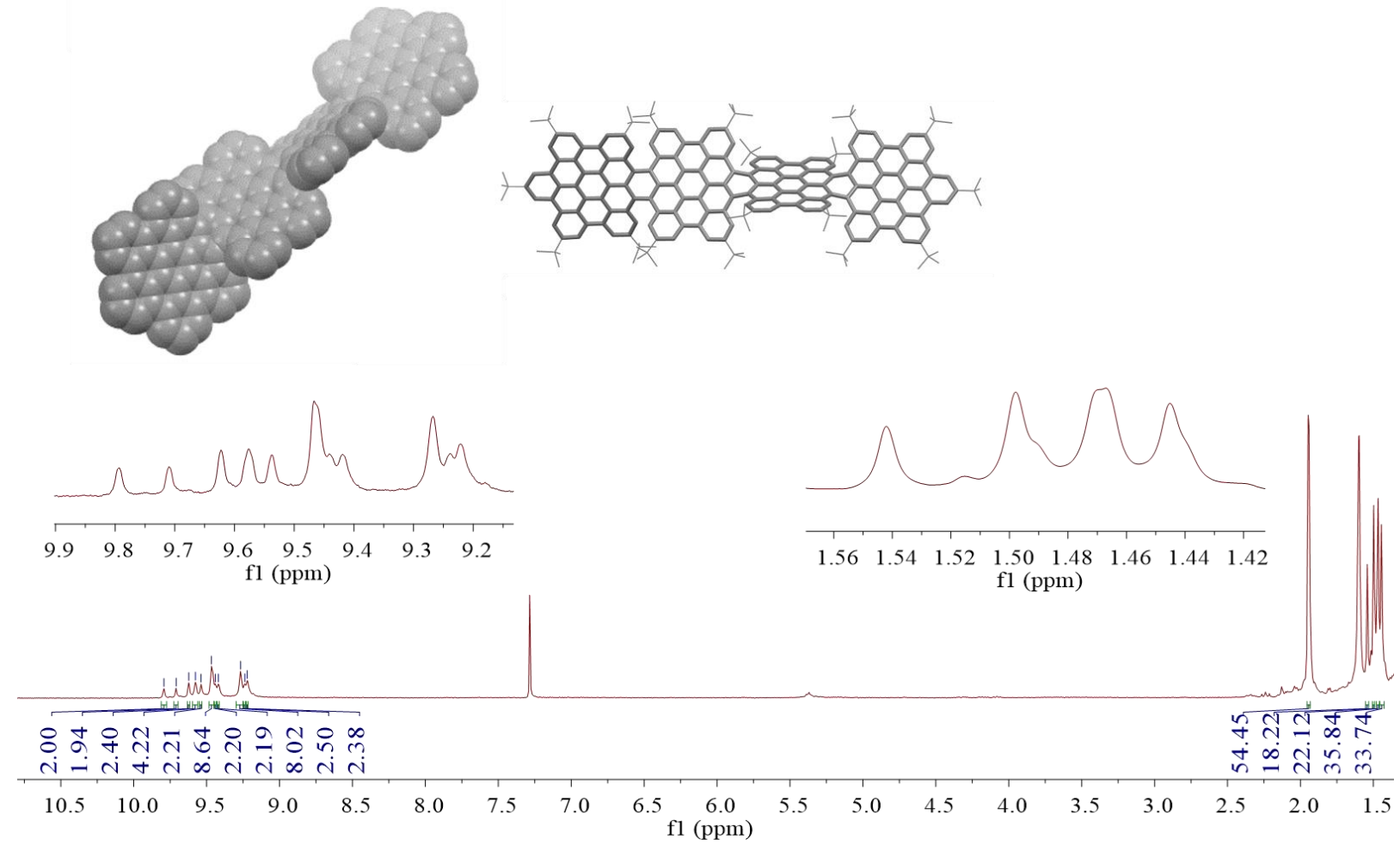

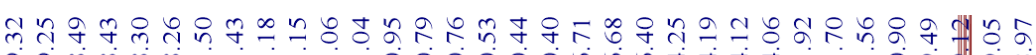

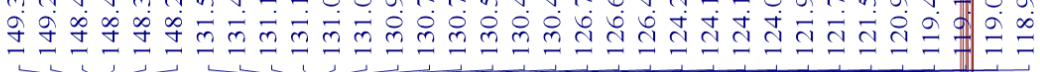

$\infty \underset{\infty}{\infty} \stackrel{m}{2}$

ले लेले ल
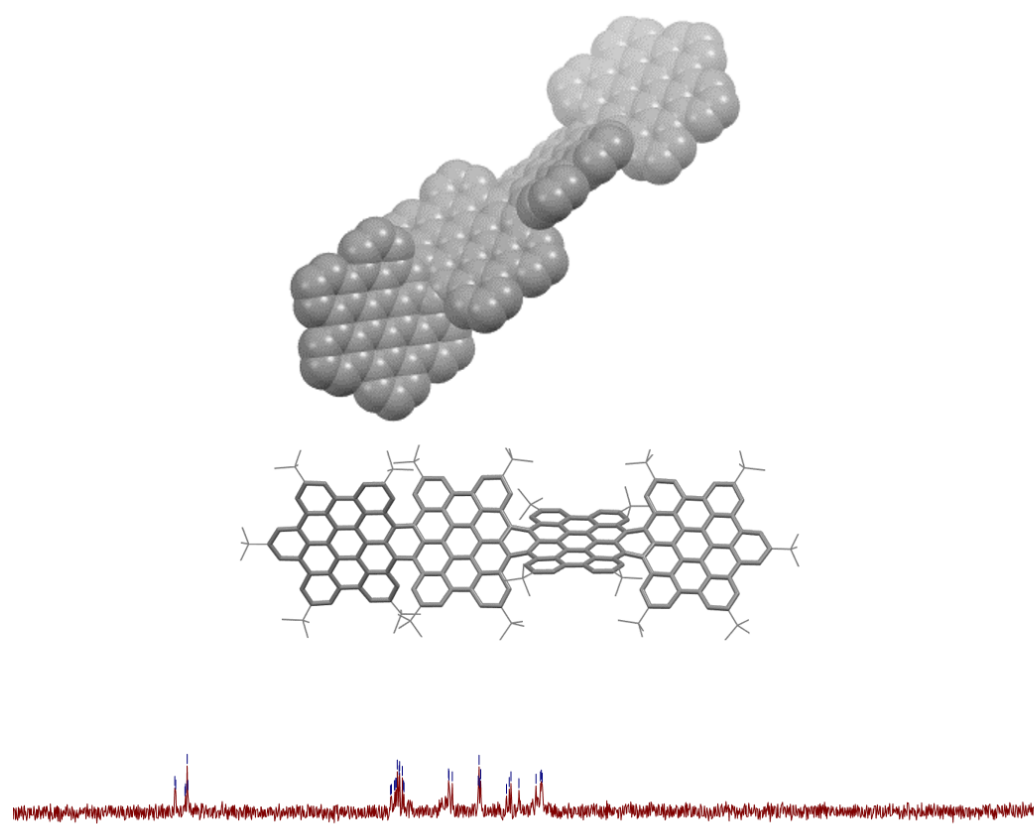

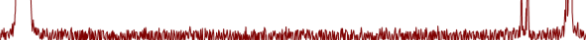

$\begin{array}{llllllllllllllllllllllllll}160 & 155 & 150 & 145 & 140 & 135 & 130 & 125 & 120 & 115 & 110 & 105 & 100 & 95 & 90 & 85 & 80 & 75 & 70 & 65 & 60 & 55 & 50 & 45 & 40 & 35 \\ \mathrm{fl}(\mathrm{ppm})\end{array}$ 


\section{${ }^{1} \mathrm{H}-{ }^{1} \mathrm{H}$ NOE spectrum of Mixed-1}

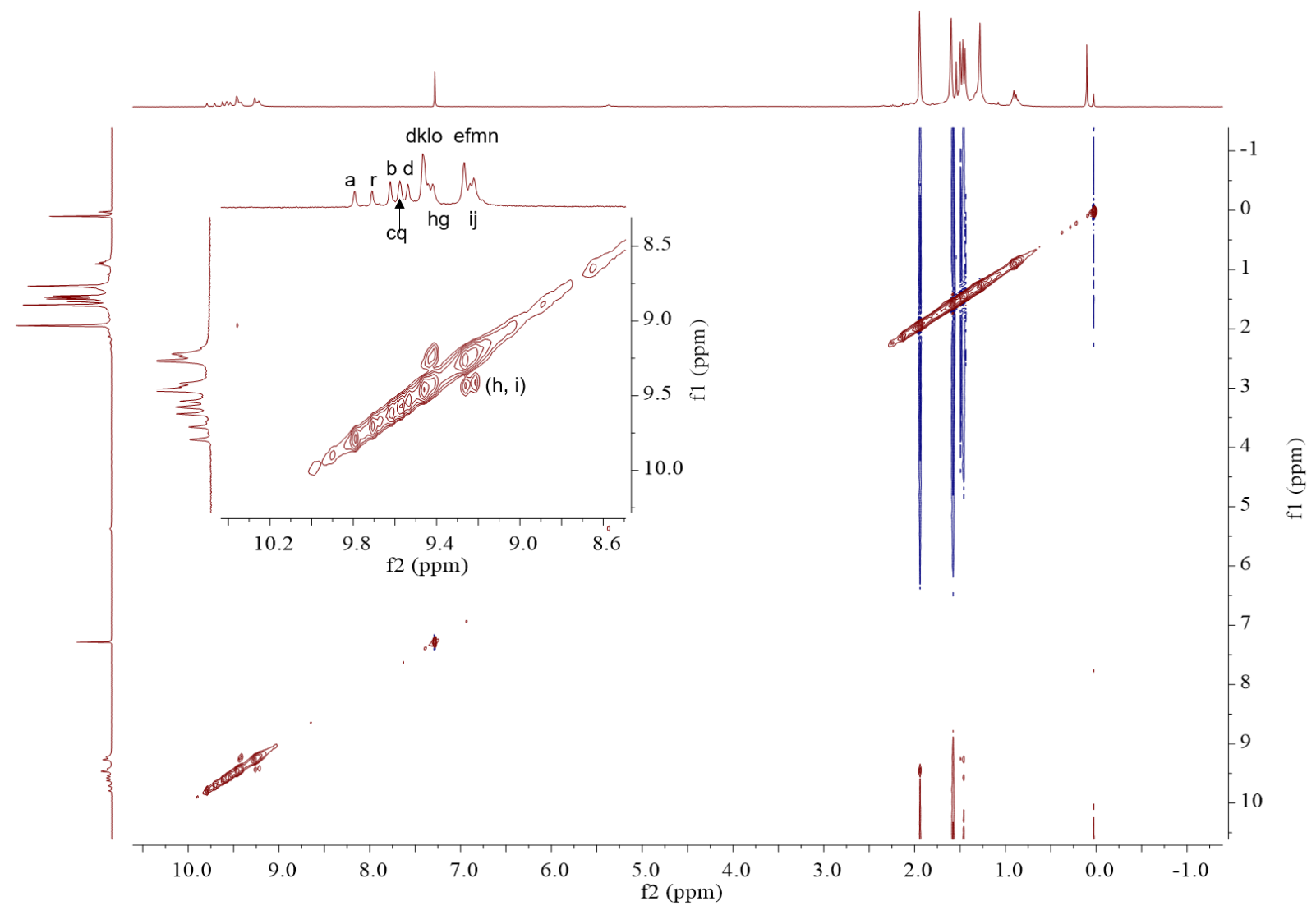

${ }^{1} \mathrm{H}-{ }^{13} \mathrm{C}$ HSQC spectrum of Mixed-1

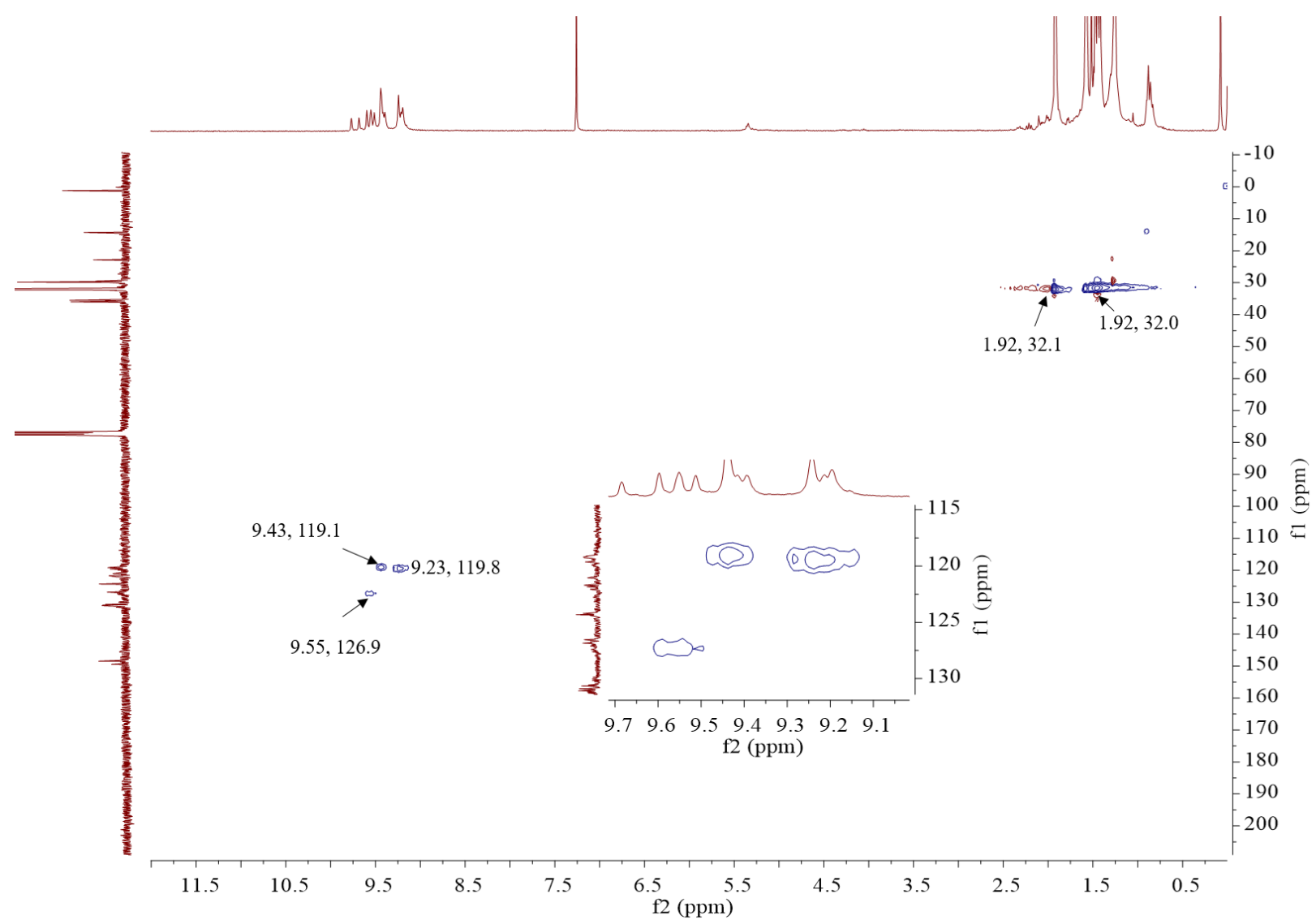


${ }^{1} \mathrm{H}-{ }^{13} \mathrm{C}$ HMBC spectrum of Mixed-1

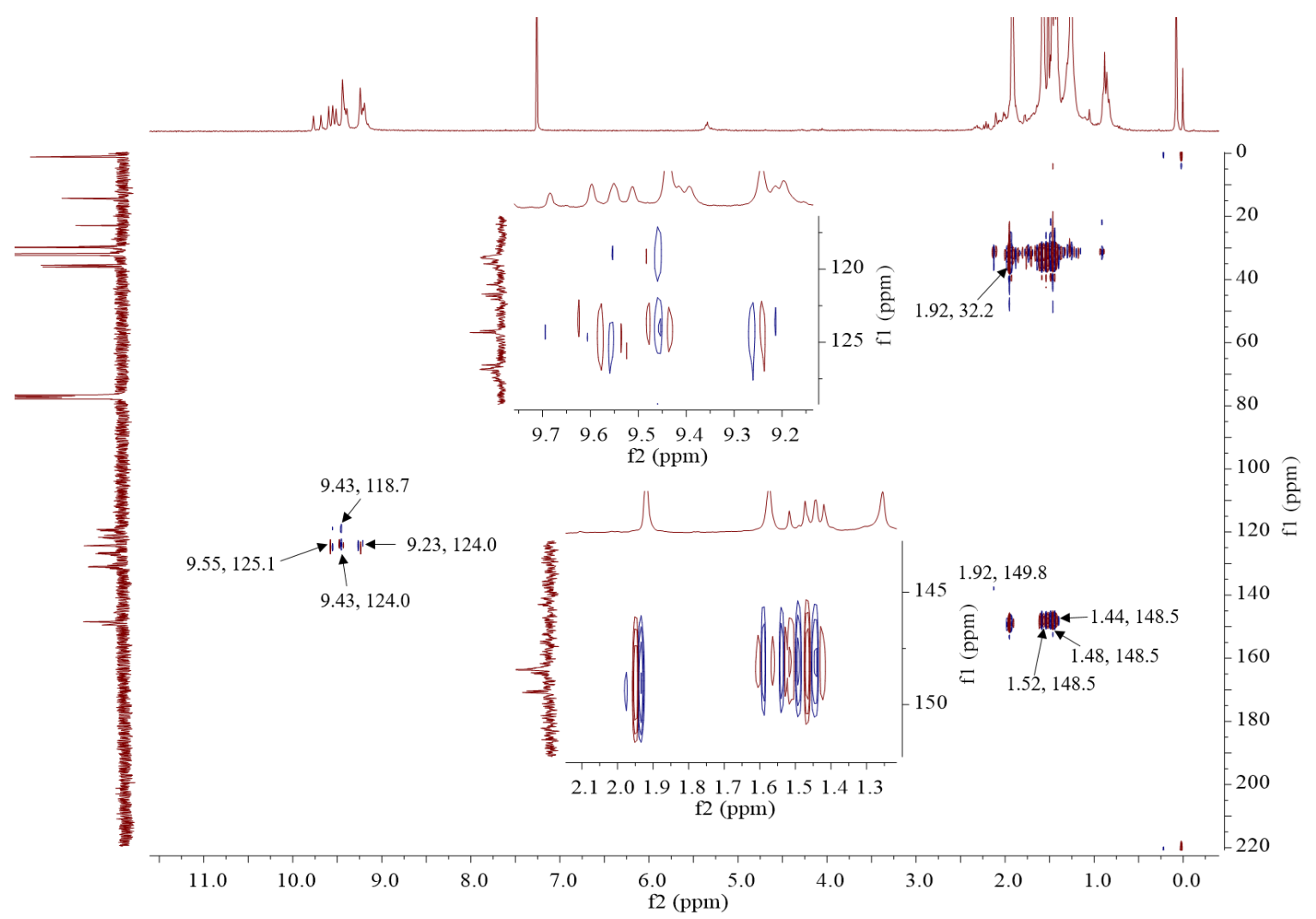


${ }^{1} \mathrm{H}$ NMR spectrum of Waggling-1

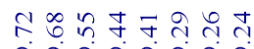

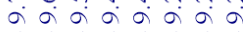

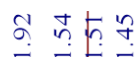

int)
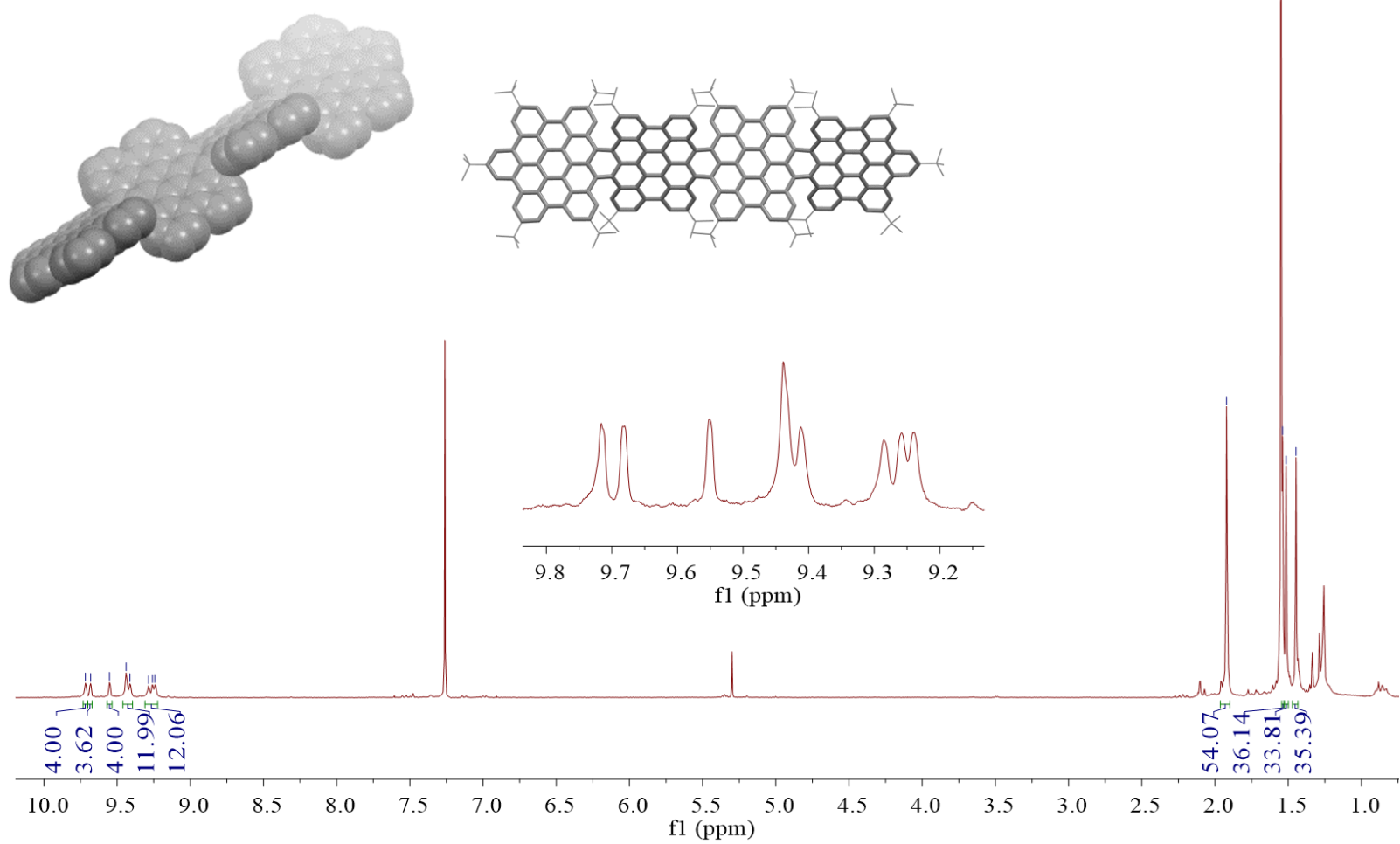


\section{${ }^{1} \mathrm{H}$ NMR and ${ }^{13} \mathrm{C}$ NMR spectra of 2}

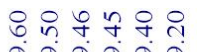

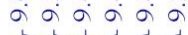
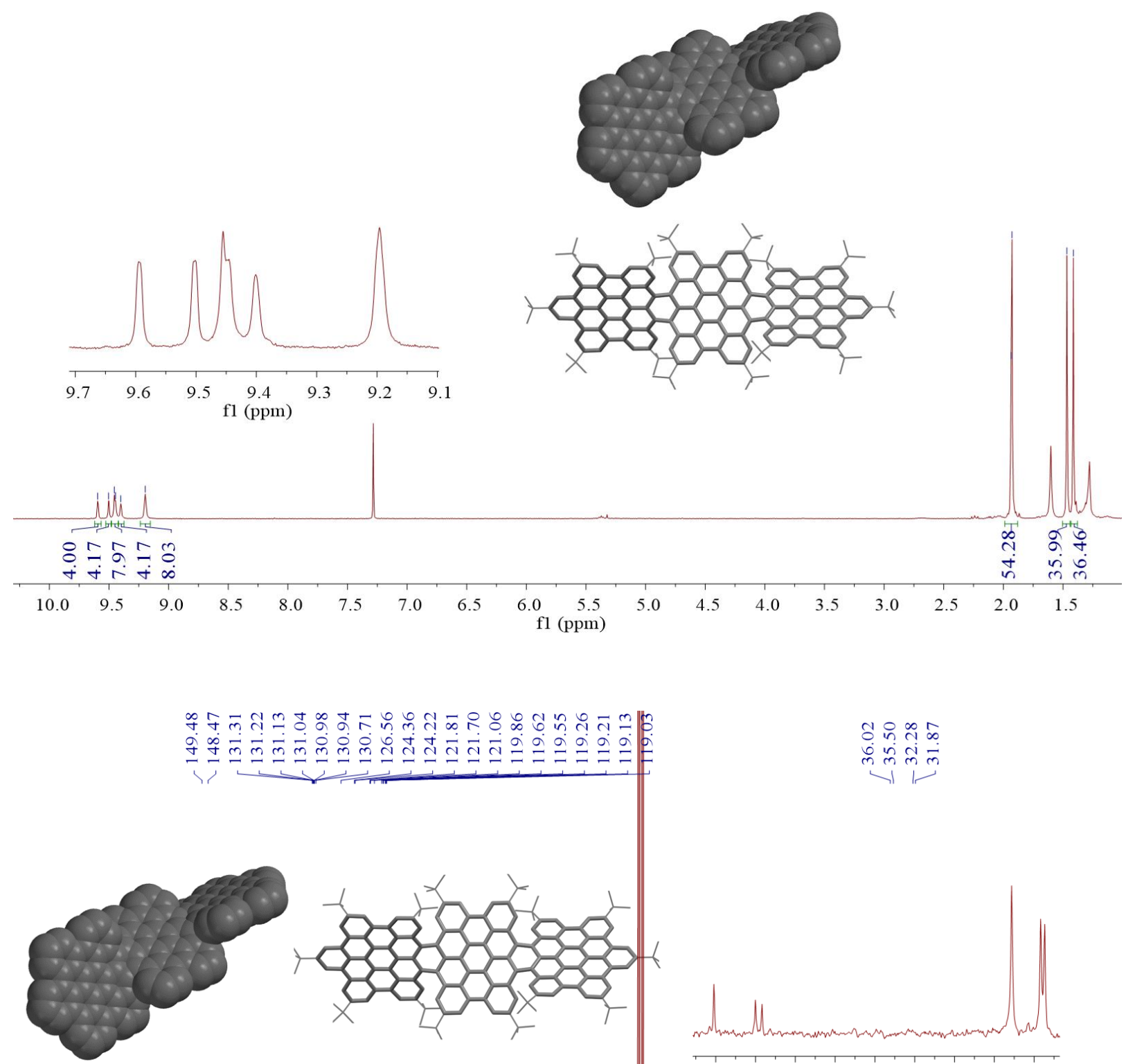

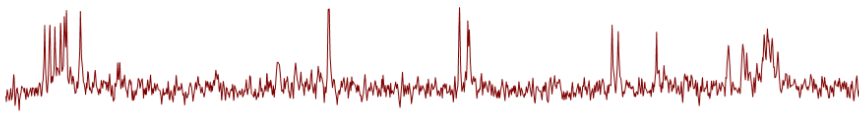

$\begin{array}{llllllllllllll}131 & 130 & 129 & 128 & 127 & 126 & 125 & 124 & 123 & 122 & 121 & 120 & 119 & 118\end{array}$ f1 (ppm)

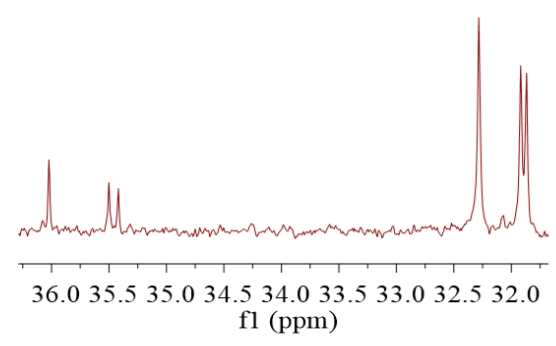

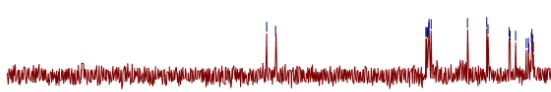


${ }^{1} \mathrm{H}$ NMR and ${ }^{13} \mathrm{C}$ NMR spectra of 17

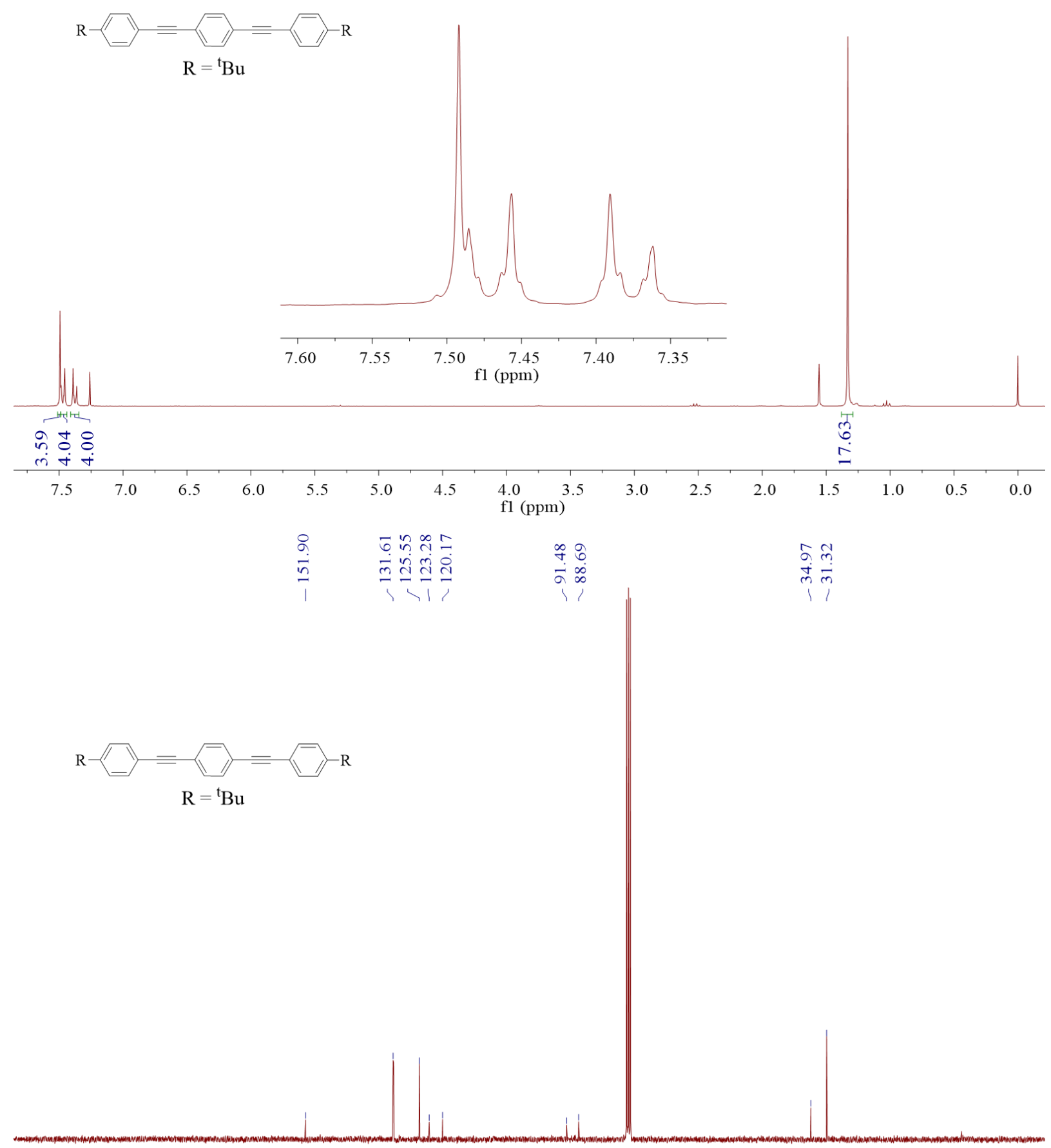

$\begin{array}{llllllllllllllllllllllllll}210 & 200 & 190 & 180 & 170 & 160 & 150 & 140 & 130 & 120 & 110 & 100 & 90 & 80 & 70 & 60 & 50 & 40 & 30 & 20 & 10 & 0 & -10\end{array}$ 


\section{${ }^{1} \mathrm{H}$ NMR and ${ }^{13} \mathrm{C}$ NMR spectra of 18}

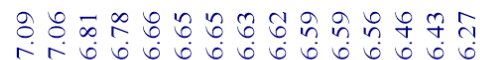

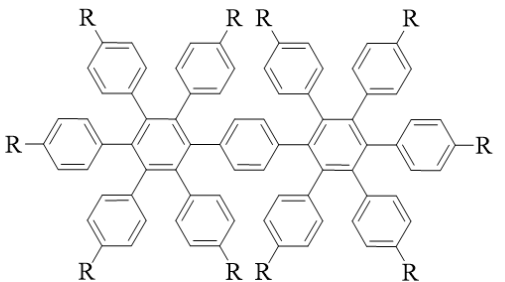

$\mathrm{R}={ }^{\mathrm{t}} \mathrm{Bu}$

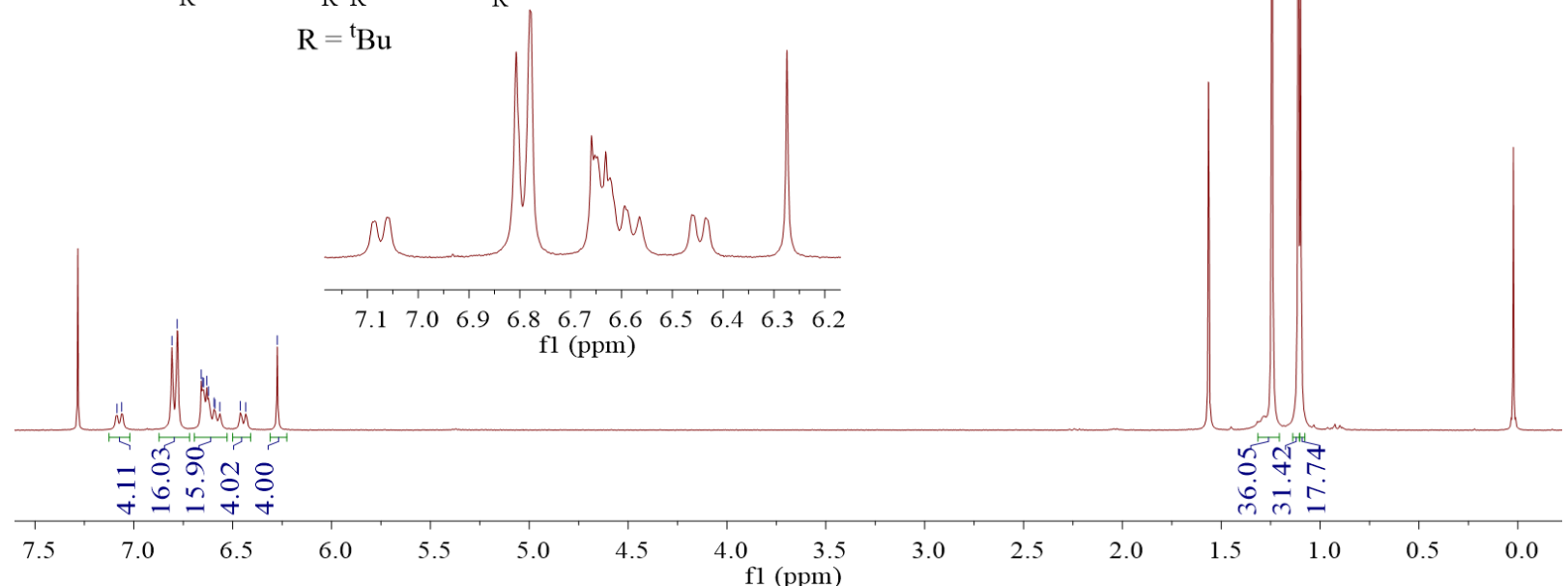

웅ำ

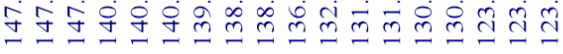
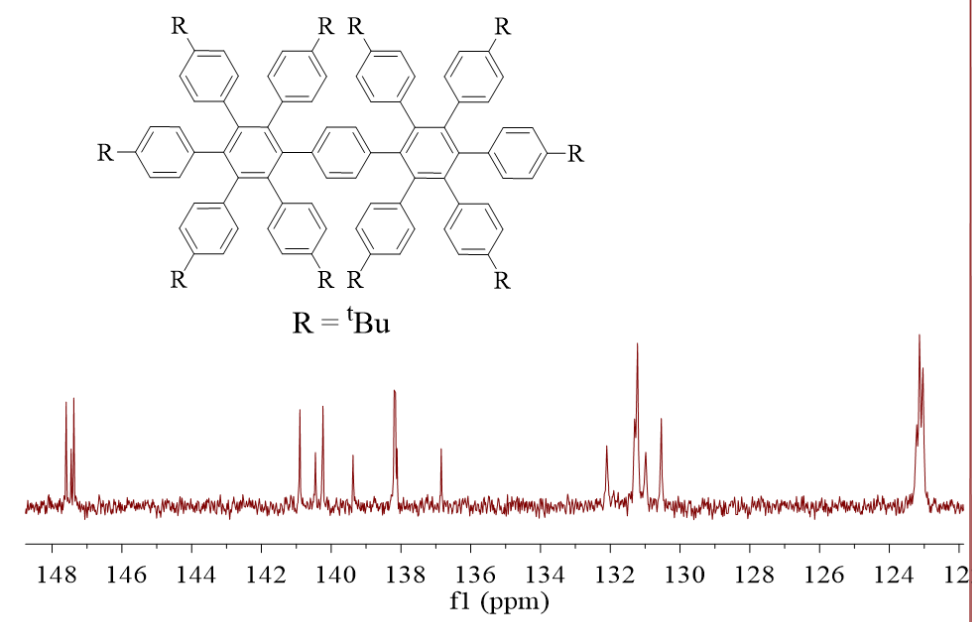

요욤ำ

迥芦向

लिख

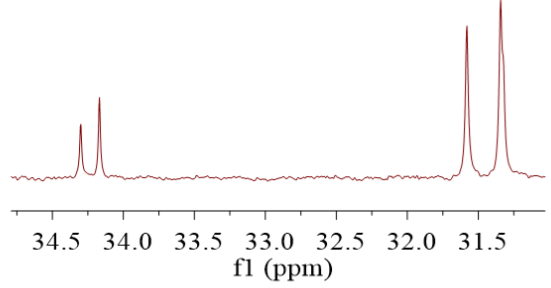


${ }^{1} \mathrm{H}$ NMR and ${ }^{13} \mathrm{C}$ NMR spectra of 3

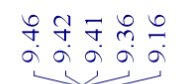

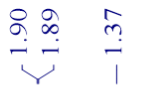
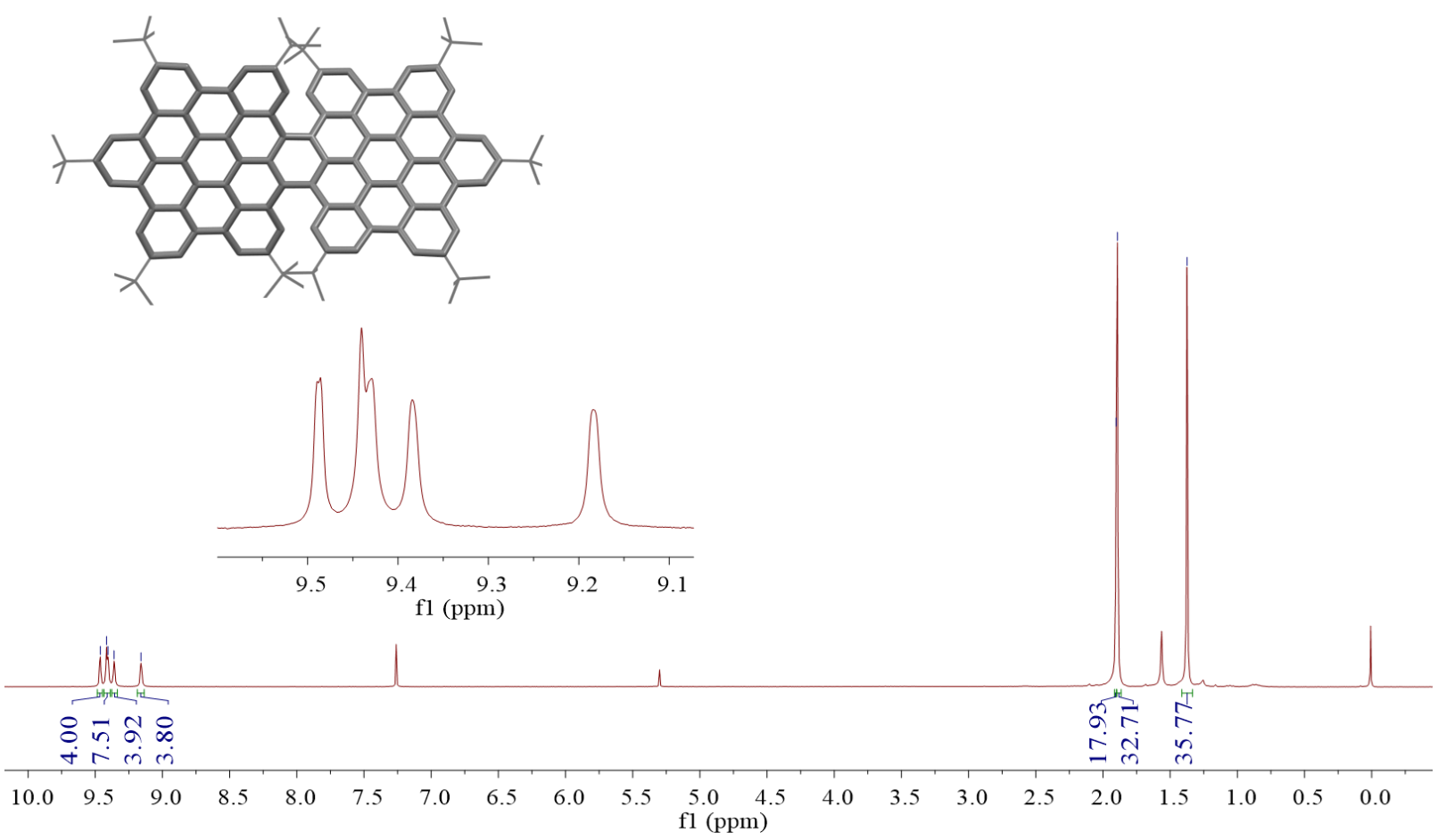

nᄄ

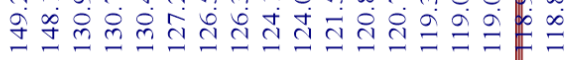

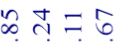

mmm
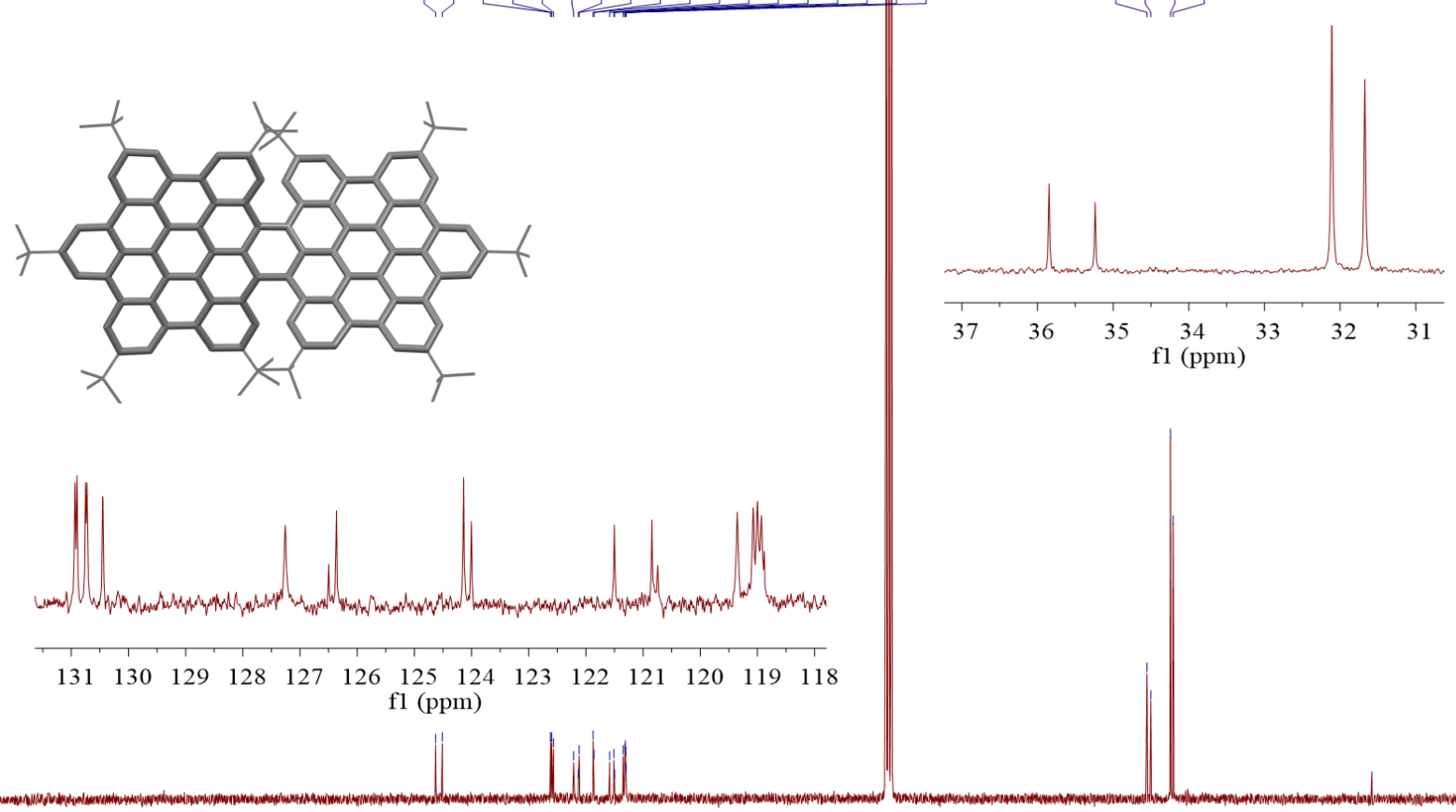

$\begin{array}{lllllllllllllllllllllll}210 & 200 & 190 & 180 & 170 & 160 & 150 & 140 & 130 & 120 & 110 & 100 & 90 & 80 & 70 & 60 & 50 & 40 & 30 & 20 & 10 & 0 & -10\end{array}$ 


\section{${ }^{1}$ H-1 H ROE spectrum of 3}

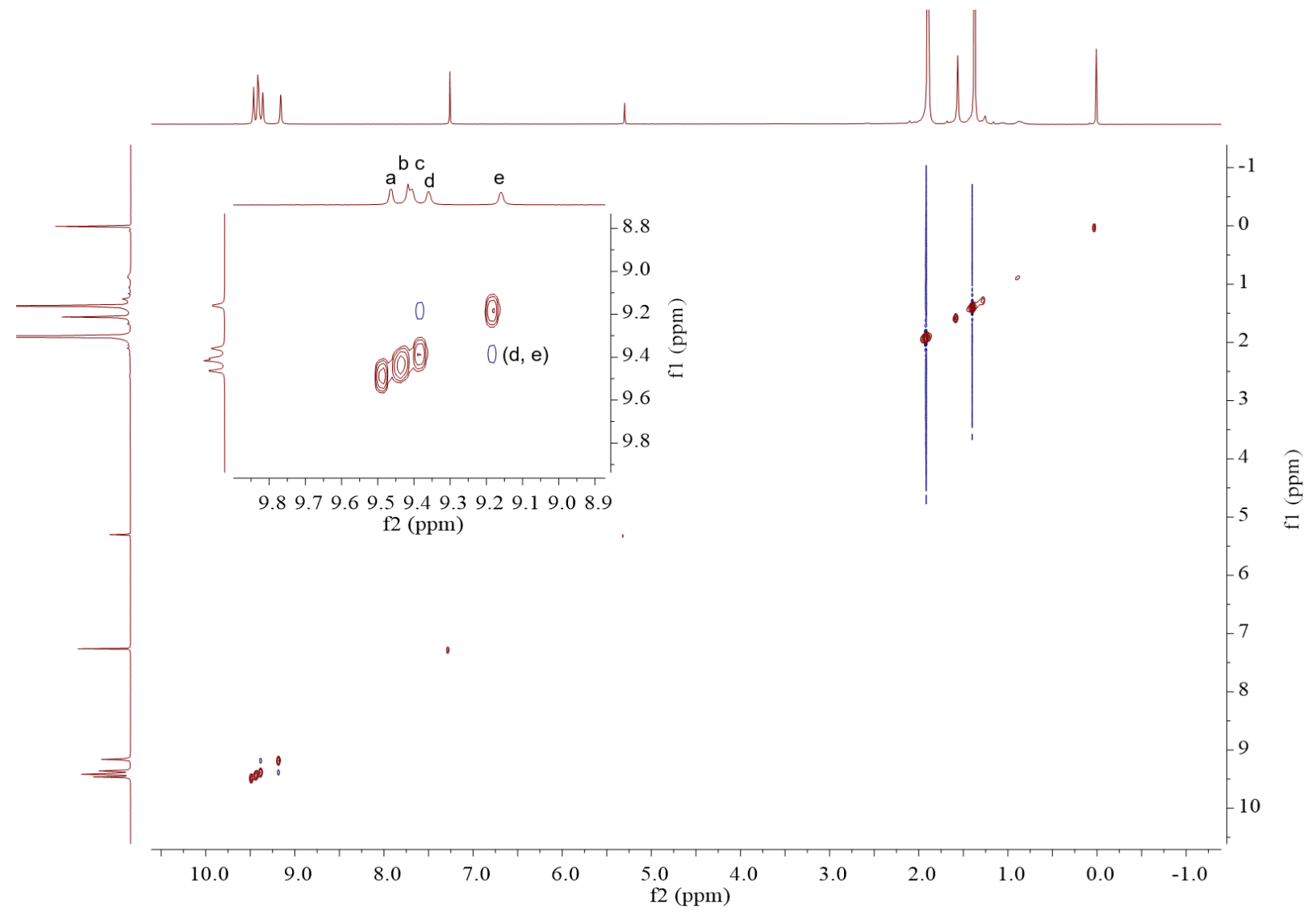

${ }^{1} \mathrm{H}-{ }^{13} \mathrm{C}$ HSQC spectrum of 3

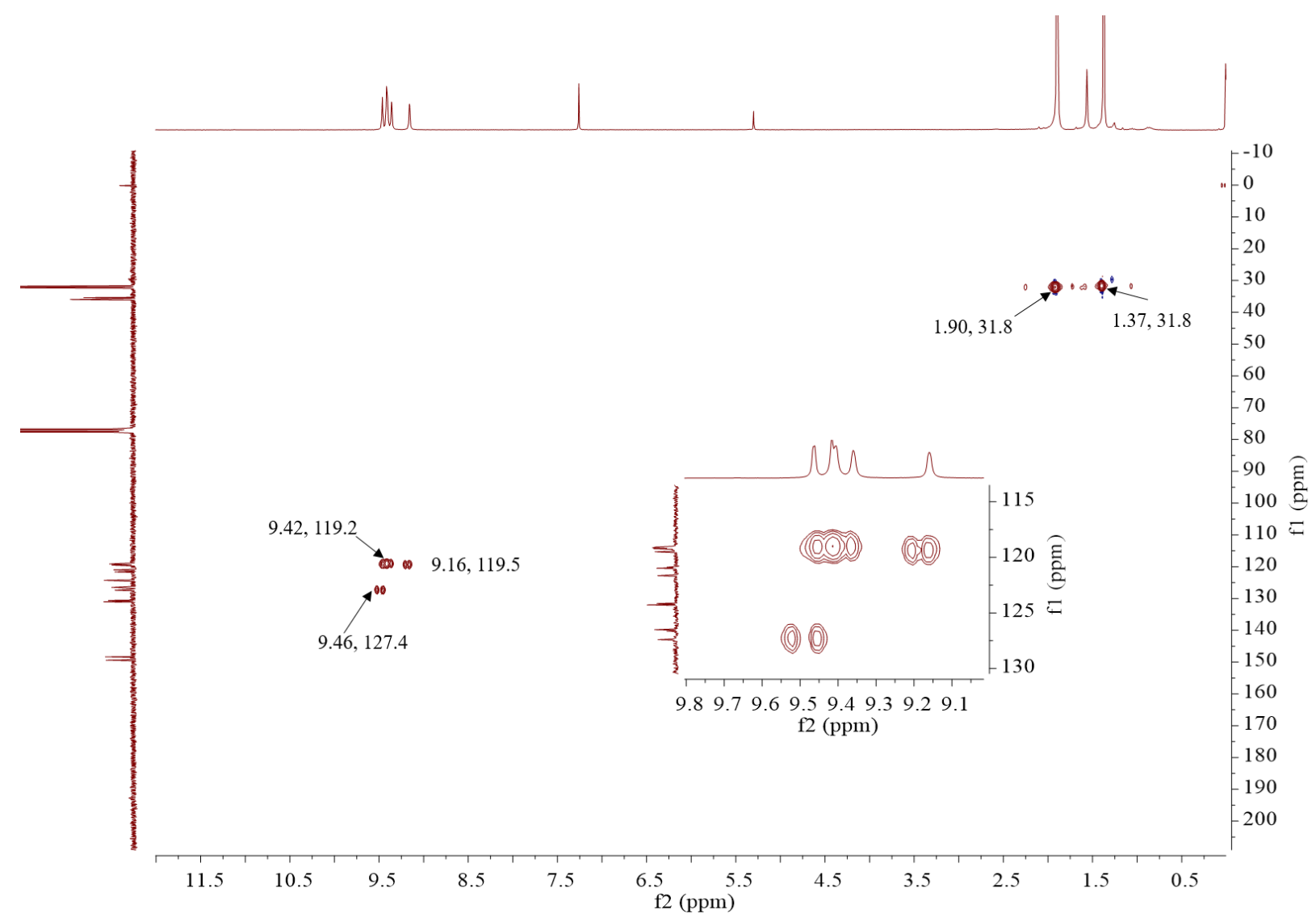


${ }^{1} \mathrm{H}-{ }^{13} \mathrm{C}$ HMBC spectrum of 3

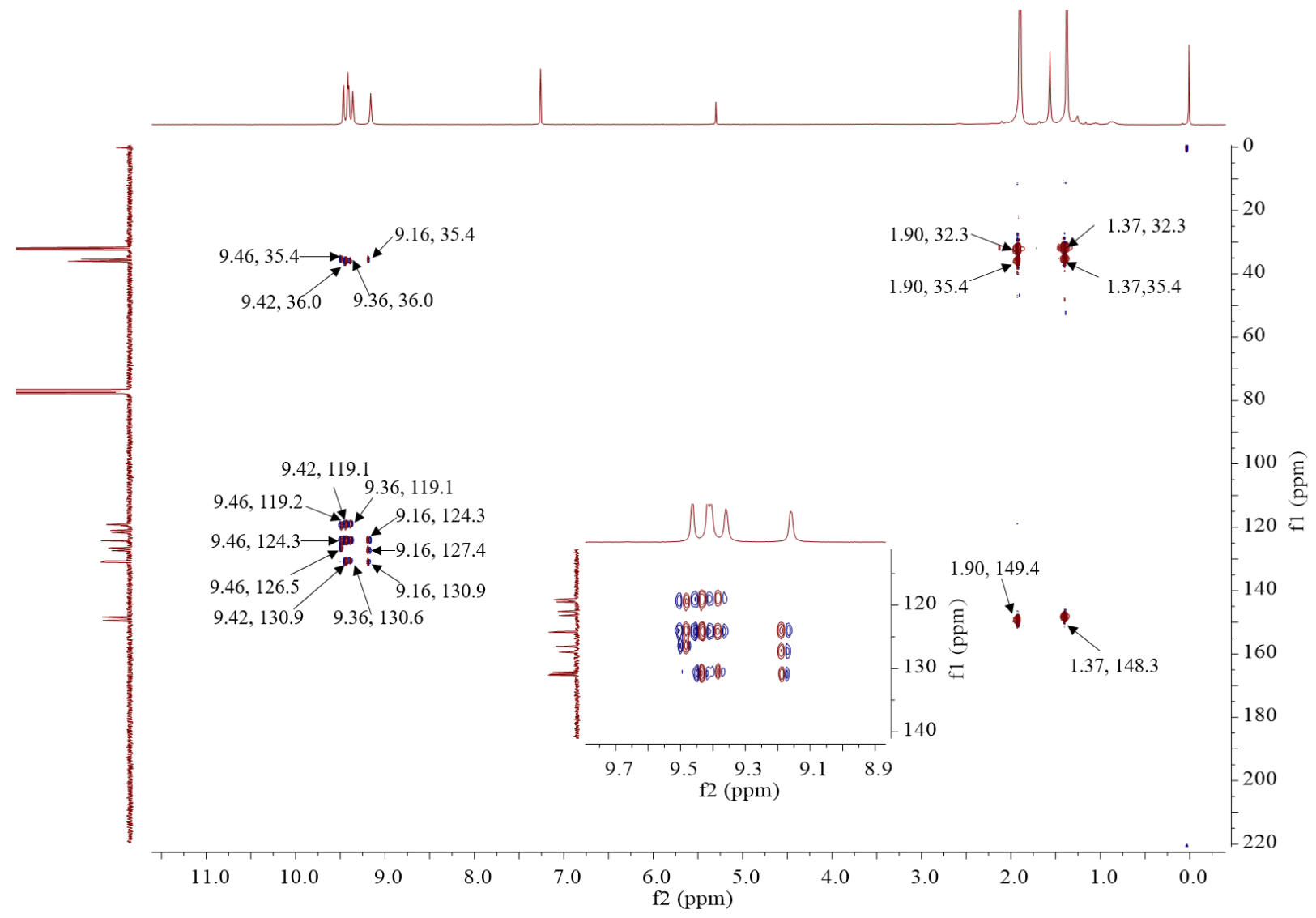


MALDI-TOF mass spectrum of 1 (helical form)

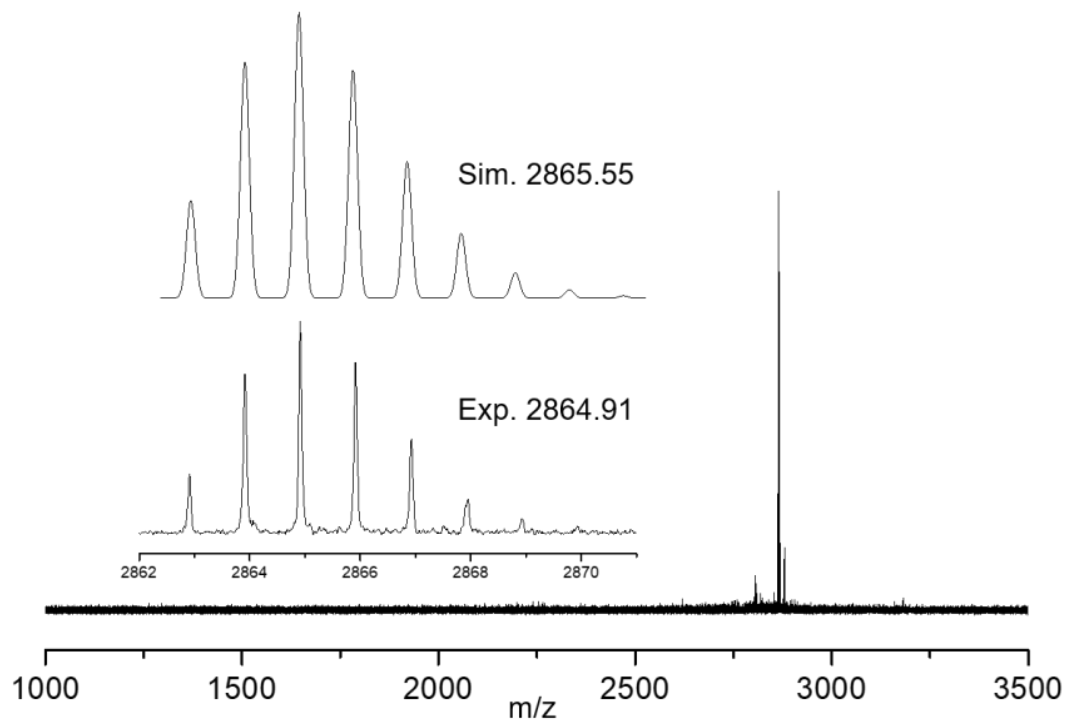

MALDI-TOF mass spectrum of Mixed-1

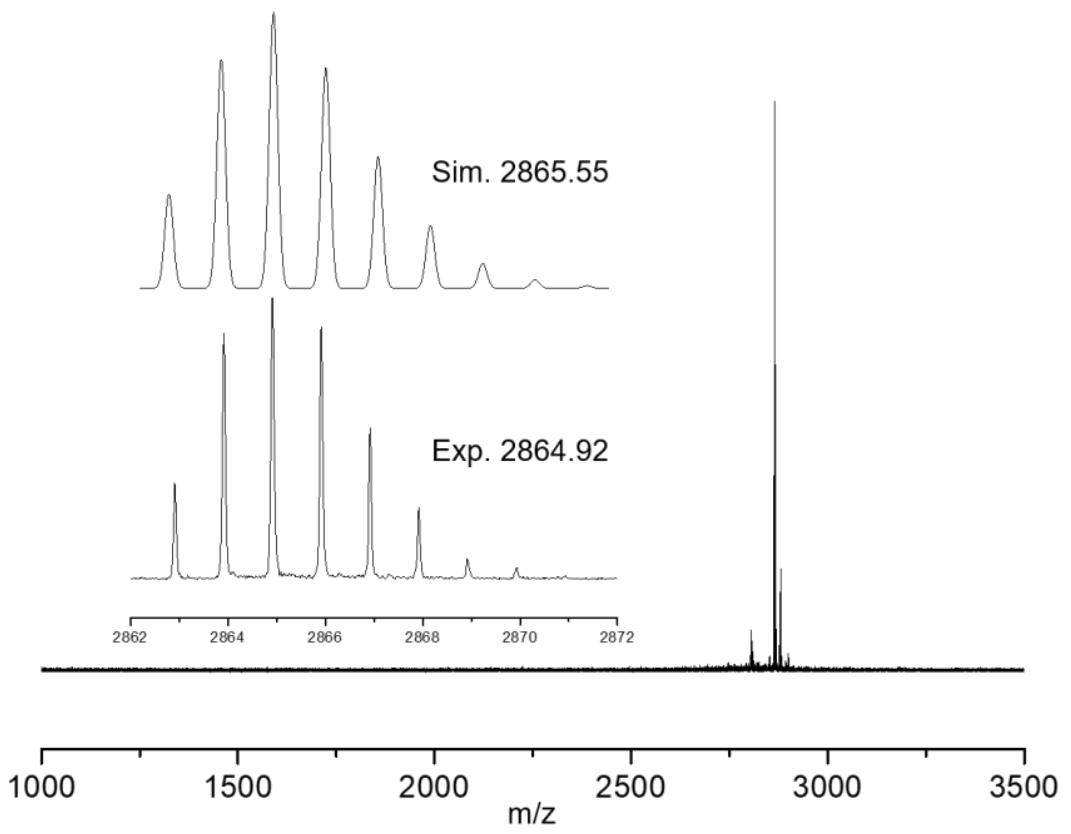


MALDI-TOF mass spectrum of Waggling-1

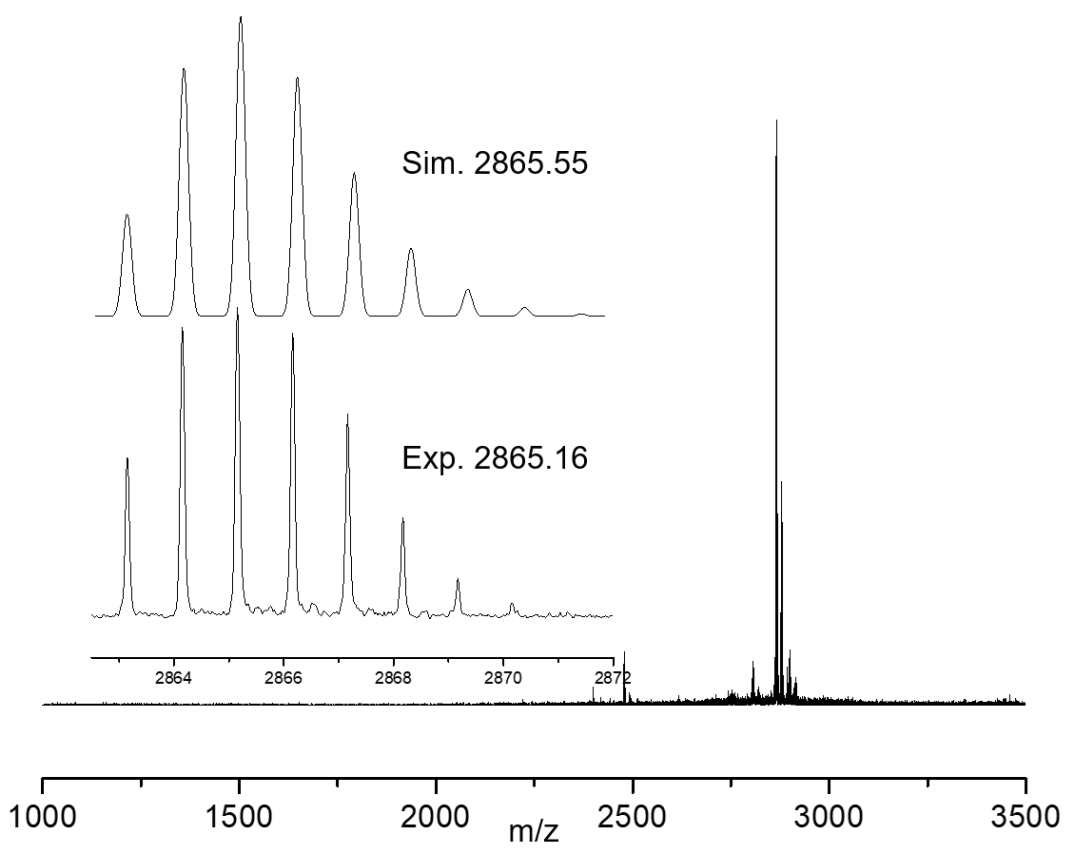


MALDI-TOF mass spectrum of 2

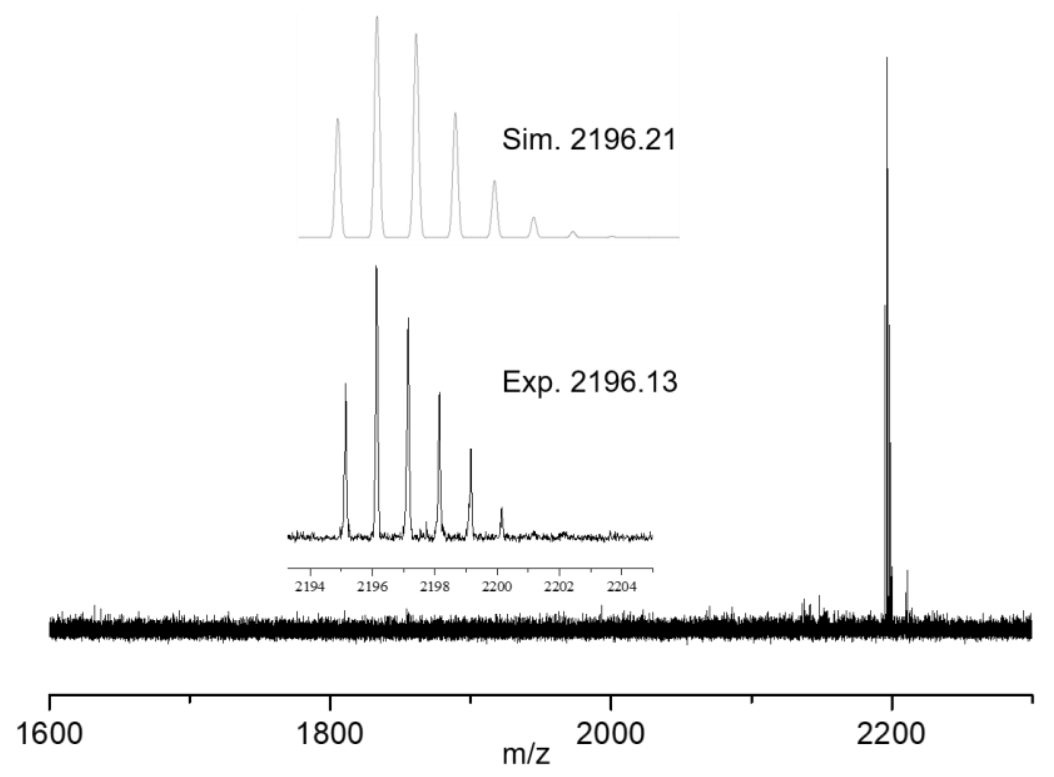

MALDI-TOF mass spectrum of meso-2

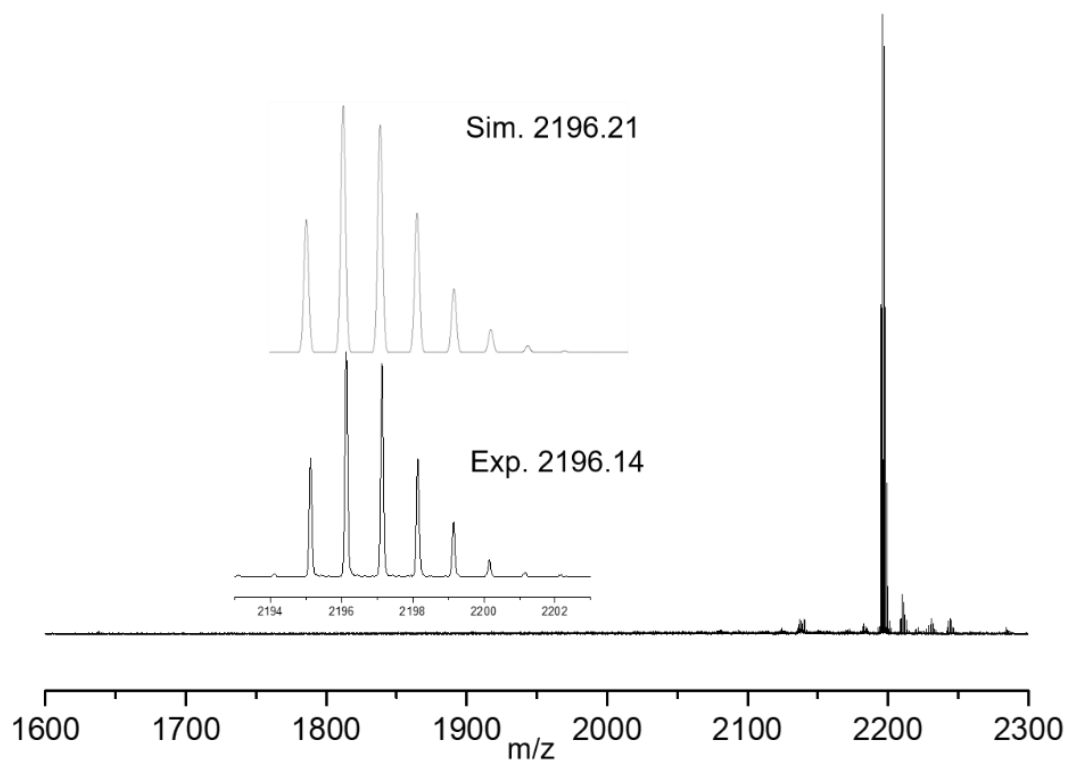


MALDI-TOF mass spectrum of 3

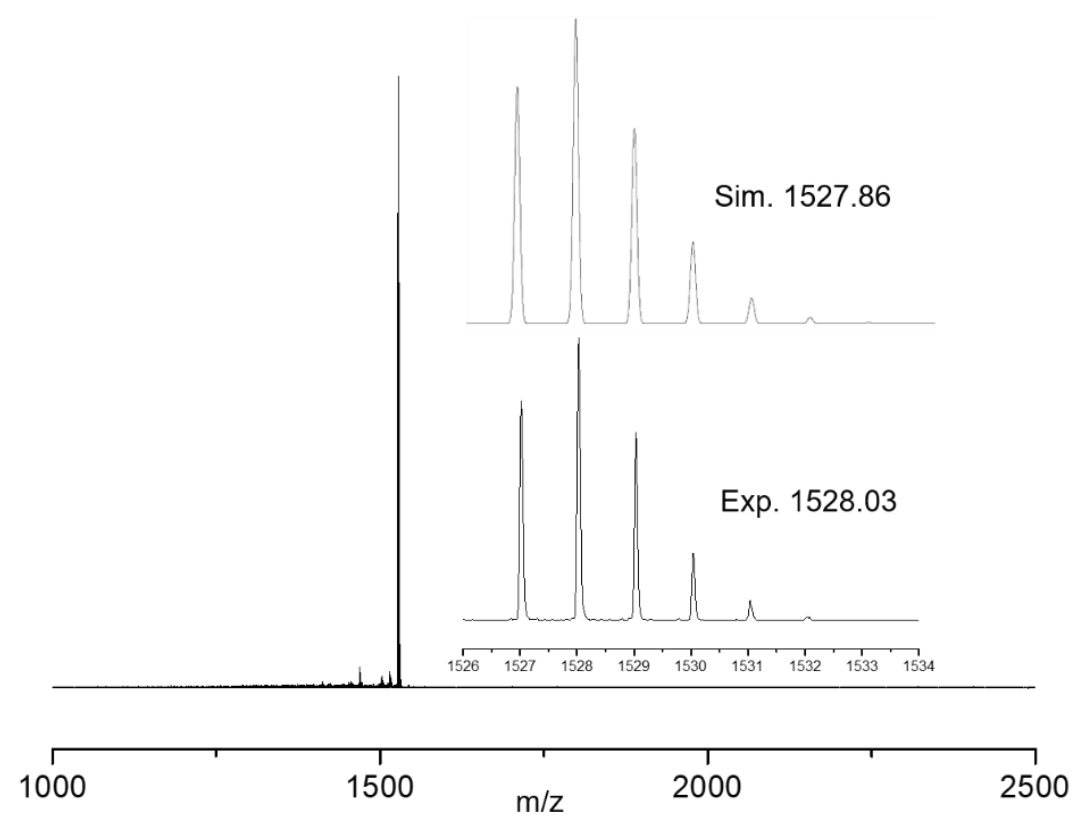




\section{References}

(1). Gaussian 16, Revision A.03, Frisch M. J.; Trucks G. W.; Schlegel H. B.; Scuseria G. E.; Robb M. A.; Cheeseman J. R.; Scalmani G.; Barone V.; Petersson G. A.; Nakatsuji H.; Li X.; Caricato M.; Marenich A. V.; Bloino J.; Janesko B. G.; Gomperts R.; Mennucci B.; Hratchian H. P.; Ortiz J. V.; Izmaylov A. F.; Sonnenberg J. L.; Williams-Young D.; Ding F.; Lipparini F.; Egidi F.; Goings J.; Peng B.; Petrone A.; Henderson T.; Ranasinghe D.; Zakrzewski V. G.; Gao J.; Rega N.; Zheng G.; Liang W.; Hada M.; Ehara M.; Toyota K.; Fukuda R.; Hasegawa J.; Ishida M.; Nakajima T.; Honda Y.; Kitao O.; Nakai H.; Vreven T.; Throssell K.; Montgomery, Jr. J. A.; Peralta J. E.; Ogliaro F.; Bearpark M. J.; Heyd J. J.; Brothers E. N.; Kudin K. N.; Staroverov V. N.; Keith T. A.; Kobayashi R.; Normand J.; Raghavachari K.; Rendell A. P.; Burant J. C.; Iyengar S. S.; Tomasi J.; Cossi M.; Millam J. M.; Klene M.; Adamo C.; Cammi R.; Ochterski J. W.; Martin R. L.; Morokuma K.; Farkas O.; Foresman J. B.; Fox D. J. Gaussian, Inc., Wallingford CT, 2016.

(2). Martin R. H.; Marchant M. J. Thermal racemisation of hepta-, octa-, and nonahelicene : Kinetic results, reaction path and experimental proofs that the racemisation of hexa- and heptahelicene does not involve an intramolecular double diels-alder reaction. Tetrahedron 1974, 30, 347-349. 